Supporting Information

\title{
Reaction of a Molybdenum Bis(dinitrogen) Complex with Carbon Dioxide: A Combined Experimental and Computational Investigation
}

Raja Pal, ${ }^{a}$ Suyeon Kim, ${ }^{b, c}$ Woojong Lee,${ }^{b, c}$ Matthew R. Mena, ${ }^{a}$ Afshan Khurshid, ${ }^{a}$ Chandrani Ghosh, ${ }^{a}$ Thomas L. Groy ${ }^{a}$ Andrew V. G. Chizmeshya, ${ }^{* a}$ Mu-Hyun Baik, ${ }^{*, c, b}$ Ryan J. Trovitch $^{*, a}$

${ }^{a}$ School of Molecular Sciences, Arizona State University, Tempe, Arizona 85287, USA.

${ }^{b}$ Department of Chemistry, Korea Advanced Institute of Science and Technology (KAIST), Daejeon 34141, Republic of Korea.

${ }^{c}$ Center for Hydrocarbon Functionalizations, Institute for Basic Science (IBS), Daejeon 34141, Republic of Korea.

*e-mail: chizmesh@asu.edu,mbaik2805@kaist.ac.kr,ryan.trovitch@asu.edu 


\section{CRYSTALLOGRAPHIC DATA}

Table S1. Crystallographic Data for $\left.\left({ }^{\mathrm{Ph} 2 \mathrm{PP}} \mathrm{DI}\right) \mathrm{Mo}(\mathrm{CO})\right)_{2}(\mathbf{1}),\left({ }^{\mathrm{Ph} 2 \mathrm{PP}} \mathrm{DI}\right) \mathrm{MoI}_{2}(\mathbf{2})$, and $\left({ }^{\mathrm{Ph} 2 \mathrm{PPr}} \mathrm{DI}\right) \mathrm{Mo}\left(\mathrm{N}_{2}\right)_{2}(\mathbf{3})$.

\begin{tabular}{|c|c|c|c|}
\hline & 1 & 2 & 3 \\
\hline chemical formula & $\begin{array}{c}2\left(\mathrm{C}_{36} \mathrm{H}_{38} \mathrm{MoN}_{2} \mathrm{O}_{2} \mathrm{P}_{2}\right) \\
1.5\left(\mathrm{C}_{6} \mathrm{H}_{6}\right)\end{array}$ & $\mathrm{C}_{34} \mathrm{H}_{38} \mathrm{I}_{2} \mathrm{MoN}_{2} \mathrm{P}_{2}$ & $\begin{array}{c}\mathrm{C}_{34} \mathrm{H}_{38} \mathrm{MoN}_{6} \mathrm{P}_{2}, \\
2\left(\mathrm{C}_{4} \mathrm{H}_{8} \mathrm{O}\right)\end{array}$ \\
\hline formula weight & 1494.28 & 886.34 & 832.79 \\
\hline crystal dimensions & $0.142 \times 0.089 \times 0.028$ & $0.153 \times 0.096 \times 0.016$ & $0.430 \times 0.230 \times 0.030$ \\
\hline crystal system & triclinic & monoclinic & monoclinic \\
\hline space group & $\mathrm{P}-1$ & P $121 / \mathrm{c} 1$ & P $121 /$ n 1 \\
\hline$a(\AA)$ & $9.1902(4)$ & $13.3035(19)$ & $11.6851(4)$ \\
\hline$b(\AA)$ & $17.1648(7)$ & $13.4768(19)$ & $23.3778(8)$ \\
\hline$c(\AA)$ & $23.2175(10)$ & $18.612(3)$ & $14.7978(5)$ \\
\hline$\alpha(\mathrm{deg})$ & $93.6680(10)$ & 90 & 90 \\
\hline$\beta(\operatorname{deg})$ & $92.9430(10)$ & $94.5078(17)$ & $99.3570(10)$ \\
\hline$\gamma(\mathrm{deg})$ & $103.0680(10)$ & 90 & 90 \\
\hline $\mathrm{V}\left(\AA^{3}\right)$ & $3552.2(3)$ & $3326.6(8)$ & $3988.6(2)$ \\
\hline $\mathrm{Z}$ & 2 & 4 & 4 \\
\hline $\mathrm{T}\left({ }^{\circ} \mathrm{C}\right)$ & $123.00(10)$ & $123.00(10)$ & $123.00(10)$ \\
\hline pcalcd $\left(\mathrm{g} \mathrm{cm}^{-3}\right)$ & 1.397 & 1.770 & 1.387 \\
\hline$\mu\left(\mathrm{mm}^{-1}\right)$ & 0.497 & 2.373 & 0.453 \\
\hline reflections collected & 28834 & 24741 & 32123 \\
\hline data/restraints/parameters & $12493 / 0 / 860$ & $5850 / 0 / 372$ & $7024 / 0 / 475$ \\
\hline $\mathrm{R}_{1}[\mathrm{I}>2 \sigma(\mathrm{I})]$ & 0.0495 & 0.0570 & 0.0338 \\
\hline $\mathrm{wR}_{2}$ (all data) & 0.1317 & 0.1491 & 0.0850 \\
\hline Goodness-of-fit & 1.002 & 1.053 & 1.090 \\
\hline Largest peak, hole $\left(\mathrm{e}^{\circ} \AA^{-3}\right)$ & $1.562,-0.468$ & $3.170,-0.790$ & $0.632,-0.508$ \\
\hline
\end{tabular}




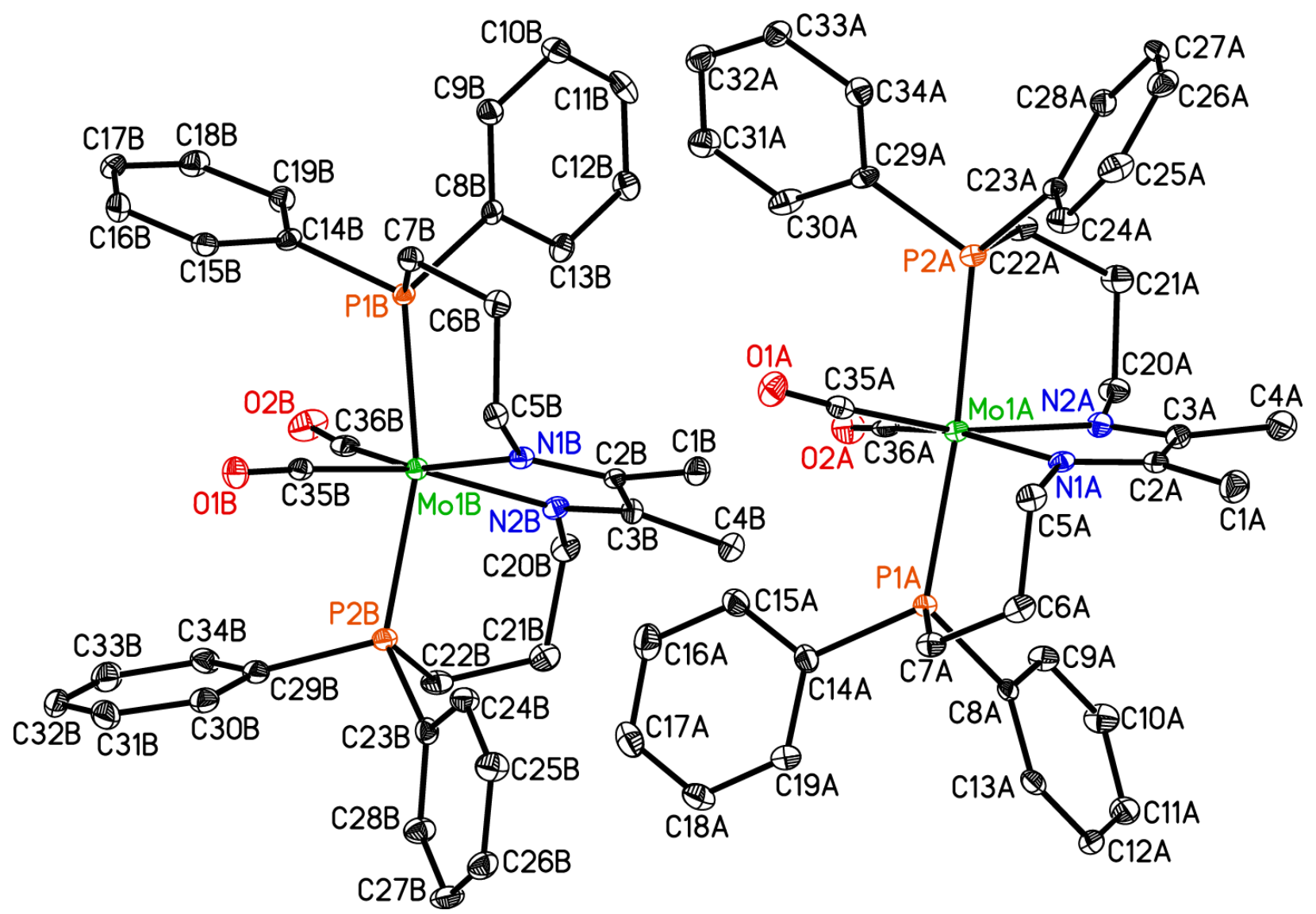

Figure S1. The molecular structure of $\mathbf{1}$ displayed at 30\% probability ellipsoids. Hydrogen atoms and co-crystallized benzene molecules omitted for clarity. 
Table S2. Bond lengths $(\AA)$ and angles $\left({ }^{\circ}\right)$ determined for $\mathbf{1}$.

\begin{tabular}{|c|c|c|c|c|c|c|}
\hline Mo1A-C36A & \multicolumn{2}{|c|}{$1.946(4)$} & C17A-C18A & $1.375(6)$ & N2B-C20B & $1.481(5)$ \\
\hline Mo1A-C35A & \multicolumn{2}{|c|}{$1.965(4)$} & C18A-C19A & $1.371(6)$ & C1B-C2B & $1.500(5)$ \\
\hline Mo1A-N2A & \multicolumn{2}{|c|}{$2.153(3)$} & C20A-C21A & $1.531(6)$ & $\mathrm{C} 2 \mathrm{~B}-\mathrm{C} 3 \mathrm{~B}$ & $1.409(5)$ \\
\hline Mo1A-N1A & \multicolumn{2}{|c|}{$2.164(3)$} & $\mathrm{C} 21 \mathrm{~A}-\mathrm{C} 22 \mathrm{~A}$ & $1.526(6)$ & $\mathrm{C} 3 \mathrm{~B}-\mathrm{C} 4 \mathrm{~B}$ & $1.513(5)$ \\
\hline Mo1A-P2A & \multicolumn{2}{|c|}{$2.4431(11)$} & $\mathrm{C} 23 \mathrm{~A}-\mathrm{C} 24 \mathrm{~A}$ & $1.392(6)$ & C5B-C6B & $1.534(5)$ \\
\hline Mo1A-P1A & \multicolumn{2}{|c|}{$2.4519(11)$} & C23A-C28A & $1.396(5)$ & C6B-C7B & $1.536(5)$ \\
\hline P1A-C7A & \multicolumn{2}{|c|}{$1.845(4)$} & C24A-C25A & $1.385(6)$ & C8B-C13B & $1.388(5)$ \\
\hline P1A-C14A & 1.847 & & $\mathrm{C} 25 \mathrm{~A}-\mathrm{C} 26 \mathrm{~A}$ & 75(6) & C8B-C9B & $9(6)$ \\
\hline P1A-C8A & 1.856 & & $\mathrm{C} 26 \mathrm{~A}-\mathrm{C} 27 \mathrm{~A}$ & $72(6)$ & C9B-C10B & $1(6)$ \\
\hline P2A-C29A & 1.837 & & C27A-C28A & $36(6)$ & C10B-C11B & $1(6)$ \\
\hline $\mathrm{P} 2 \mathrm{~A}-\mathrm{C} 23 \mathrm{~A}$ & 1.838 & & C29A-C30A & 78(6) & $\mathrm{C} 11 \mathrm{~B}-\mathrm{C} 12 \mathrm{~B}$ & $2(6)$ \\
\hline $\mathrm{P} 2 \mathrm{~A}-\mathrm{C} 22 \mathrm{~A}$ & 1.852 & & C29A-C34A & $9(6)$ & C12B-C13B & $4(6)$ \\
\hline O1A-C35A & 1.175 & & C30A-C31A & $0(6)$ & C14B-C15B & $4(6)$ \\
\hline $\mathrm{O} 2 \mathrm{~A}-\mathrm{C} 36 \mathrm{~A}$ & 1.181 & & C31A-C32A & $77(6)$ & C14B-C19B & $4(5)$ \\
\hline N1A-C2A & 1.325 & & C32A-C33A & 79(6) & C15B-C16B & $1(6)$ \\
\hline N1A-C5A & 1.471 & & C33A-C34A & $31(6)$ & C16B-C17B & $8(6)$ \\
\hline $\mathrm{N} 2 \mathrm{~A}-\mathrm{C} 3 \mathrm{~A}$ & 1.327 & & Mo1B-C36B & $57(4)$ & C17B-C18B & $4(6)$ \\
\hline $\mathrm{N} 2 \mathrm{~A}-\mathrm{C} 20 \mathrm{~A}$ & 1.468 & & Mo1B-C35B & 72(4) & C18B-C19B & $1(6)$ \\
\hline C1A-C2A & $1.50^{7}$ & & Mo1B-N1B & $35(3)$ & C20B-C21B & $8(6)$ \\
\hline $\mathrm{C} 2 \mathrm{~A}-\mathrm{C} 3 \mathrm{~A}$ & $1.43 \varepsilon$ & & Mo1B-N2B & $51(3)$ & C21B-C22B & $0(6)$ \\
\hline $\mathrm{C} 3 \mathrm{~A}-\mathrm{C} 4 \mathrm{~A}$ & 1.508 & & Mo1B-P2B & $68(11)$ & $\mathrm{C} 23 \mathrm{~B}-\mathrm{C} 28 \mathrm{~B}$ & $0(6)$ \\
\hline C5A-C6A & 1.532 & & Mo1B-P1B & 294(10) & C23B-C24B & $3(6)$ \\
\hline C6A-C7A & 1.535 & & P1B-C8B & $30(4)$ & C24B-C25B & $8(6)$ \\
\hline C8A-C13A & $1.38 \varepsilon$ & & P1B-C14B & $38(4)$ & $\mathrm{C} 25 \mathrm{~B}-\mathrm{C} 26 \mathrm{~B}$ & $5(6)$ \\
\hline C8A-C9A & 1.385 & & P1B-C7B & $39(4)$ & C26B-C27B & $9(6)$ \\
\hline C9A-C10A & 1.391 & & P2B-C29B & $32(4)$ & C27B-C28B & $5(6)$ \\
\hline C10A-C11A & 1.385 & & $\mathrm{P} 2 \mathrm{~B}-\mathrm{C} 23 \mathrm{~B}$ & & C29B-C $30 B$ & \\
\hline $\mathrm{C} 11 \mathrm{~A}-\mathrm{C} 12 \mathrm{~A}$ & 1.3 & & $\mathrm{P} 2 \mathrm{~B}-\mathrm{C} 22 \mathrm{~B}$ & (4) & C29B-C34B & (6) \\
\hline C12A-C13A & 1.38 & & $\mathrm{O} 1 \mathrm{~B}-\mathrm{C} 35 \mathrm{~B}$ & $59(5)$ & C30B-C $31 B$ & $4(6)$ \\
\hline C14A-C15A & 1.371 & & $\mathrm{O} 2 \mathrm{~B}-\mathrm{C} 36 \mathrm{~B}$ & $63(5)$ & C31B-C $32 B$ & 7(7) \\
\hline C14A-C19A & 1.401 & & N1B-C2B & $2(5)$ & C32B-C $33 B$ & $9(7)$ \\
\hline C15A-C16A & 1.387 & & N1B-C5B & $32(5)$ & C33B-C34B & $1(6)$ \\
\hline C16A-C17A & 1.37 & & $\mathrm{~N} 2 \mathrm{~B}-\mathrm{C} 3 \mathrm{~B}$ & $36(5)$ & & \\
\hline C36A-Mo1A & & $93.93(16)$ & C7A-P1A-C8A & $104.46(18)$ & C20A-N2A-Mo1A & $120.7(3)$ \\
\hline C36A-Mo1A & & $96.55(15)$ & C14A-P1A-C8A & $100.07(18)$ & $\mathrm{N} 1 \mathrm{~A}-\mathrm{C} 2 \mathrm{~A}-\mathrm{C} 3 \mathrm{~A}$ & $114.5(4)$ \\
\hline C35A-Mo1A & & $163.97(14)$ & C7A-P1A-Mo1A & $112.96(13)$ & N1A-C2A-C1A & $123.8(4)$ \\
\hline C36A-Mo1A & $1 \mathrm{~A}$ & $163.76(14)$ & C14A-P1A-Mo1A & $120.83(14)$ & $\mathrm{C} 3 \mathrm{~A}-\mathrm{C} 2 \mathrm{~A}-\mathrm{C} 1 \mathrm{~A}$ & $121.7(4)$ \\
\hline C35A-Mo1A & N1A & $100.29(14)$ & C8A-P1A-Mo1A & $116.45(13)$ & $\mathrm{N} 2 \mathrm{~A}-\mathrm{C} 3 \mathrm{~A}-\mathrm{C} 2 \mathrm{~A}$ & $113.4(4)$ \\
\hline N2A-Mo1A- & & $71.37(12)$ & C29A-P2A-C23A & $101.12(18)$ & $\mathrm{N} 2 \mathrm{~A}-\mathrm{C} 3 \mathrm{~A}-\mathrm{C} 4 \mathrm{~A}$ & $124.5(4)$ \\
\hline C36A-Mo1A & & $84.95(11)$ & C29A-P2A-C22A & $100.59(18)$ & $\mathrm{C} 2 \mathrm{~A}-\mathrm{C} 3 \mathrm{~A}-\mathrm{C} 4 \mathrm{~A}$ & $122.1(4)$ \\
\hline C35A-Mo1A & & $89.12(11)$ & $\mathrm{C} 23 \mathrm{~A}-\mathrm{P} 2 \mathrm{~A}-\mathrm{C} 22 \mathrm{~A}$ & $103.63(19)$ & N1A-C5A-C6A & $110.6(3)$ \\
\hline N2A-Mo1A-1 & & $79.79(9)$ & C29A-P2A-Mo1A & $120.13(13)$ & C5A-C6A-C7A & $114.4(3)$ \\
\hline N1A-Mo1A- & & $102.94(9)$ & C23A-P2A-Mo1A & $118.20(13)$ & C6A-C7A-P1A & $115.9(3)$ \\
\hline C36A-Mo1A & $1 \mathrm{~A}$ & $91.94(11)$ & C22A-P2A-Mo1A & $110.63(13)$ & C13A-C8A-C9A & $118.6(4)$ \\
\hline C35A-Mo1A & & $87.29(11)$ & C2A-N1A-C5A & $118.1(3)$ & C13A-C8A-P1A & $124.9(3)$ \\
\hline N2A-Mo1A-1 & & $104.40(9)$ & C2A-N1A-Mo1A & $119.8(3)$ & C9A-C8A-P1A & $116.5(3)$ \\
\hline N1A-Mo1A- & & $81.03(9)$ & C5A-N1A-Mo1A & $121.3(3)$ & C8A-C9A-C10A & $121.1(4)$ \\
\hline P2A-Mo1A-F & & $175.08(4)$ & C3A-N2A-C20A & $117.8(4)$ & C11A-C10A-C9A & $119.0(4)$ \\
\hline C7A-P1A-C1 & & $99.49(19)$ & C3A-N2A-Mo1A & $120.7(3)$ & C12A-C11A-C10A & $120.6(4)$ \\
\hline
\end{tabular}




\begin{tabular}{|c|c|c|c|c|c|}
\hline C11A-C12A-C13A & $120.1(4)$ & C36B-Mo1B-P2B & $84.22(11)$ & C13B-C8B-P1B & $120.5(3)$ \\
\hline C12A-C $13 \mathrm{~A}-\mathrm{C} 8 \mathrm{~A}$ & $120.7(4)$ & C35B-Mo1B-P2B & $88.02(11)$ & C9B-C8B-P1B & $121.4(3)$ \\
\hline 15A-C14A-C19A & $117.3(4)$ & 1B-Mo1B-P2B & $109.58(9)$ & C10B-C9B-C8B & $120.9(4)$ \\
\hline C15A-C14A-P1A & $120.8(3)$ & N2B-Mo1B-P2B & $81.23(9)$ & C11B-C10B-C9B & $120.0(4)$ \\
\hline C19A-C14A-P1A & $21.9(3)$ & 36B-Mo1B-P1B & $85.53(11)$ & C10B-C11B-C12B & $120.2(4)$ \\
\hline C14A-C15A-C16A & $21.6(4)$ & 35B-Mo1B-P1B & $82.01(11)$ & C11B-C12B-C13B & $119.9(4)$ \\
\hline C17A-C16A-C15A & $120.1(4)$ & N1B-Mo1B-P1B & $82.87(9)$ & C12B-C13B-C $8 B$ & $120.9(4)$ \\
\hline C16A-C17A-C18A & $119.3(4)$ & N2B-Mo1B-P1B & & C15B-C14B-C19B & \\
\hline C19A-C18A-C17A & 120.3 & 2B-Mo11 & & 15B-C14B-P1B & $6(3)$ \\
\hline C18A-C19A-C14A & 121.3 & 8B-P1B-C14B & 18) & 19B-C14B-P1B & $119.6(3)$ \\
\hline N2A-C20A-C21A & $112.0(3)$ & C8B-P1B-C7B & & C16B-C15B-C14B & $120.4(4)$ \\
\hline C22A-C21A-C20A & $115.0(4)$ & C14B-P1B-C7B & $102.86(18)$ & C17B-C16B-C15B & $120.4(4)$ \\
\hline C21A-C22A-P2A & & & & C18B-C17B-C16B & $120.1(4)$ \\
\hline C24A-C $23 \mathrm{~A}-\mathrm{C} 28 \mathrm{~A}$ & 118. & C14B-P & & C17B-C $18 B-C 19 B$ & $.3(4)$ \\
\hline C24A-C23A-P2A & 117. & $7 \mathrm{~B}-\mathrm{F}$ & & C14B & $3(4)$ \\
\hline & 124. & $\mathrm{C} 29 \mathrm{~B}$ & & $21 \mathrm{~B}$ & $.5(3)$ \\
\hline C25A-C24A-C23A & $120.6(4)$ & C29B-I & & $\mathrm{C} 20 \mathrm{~B}-\mathrm{C} 21 \mathrm{~B}-\mathrm{C} 22 \mathrm{~B}$ & $113.7(3)$ \\
\hline C26A-C25A-C24A & $20.6(4)$ & C23B- & & C21B-C22B-P2B & $113.4(3)$ \\
\hline C27A-C26A-C25A & 119.5 & C29B- & & $\mathrm{C} 24 \mathrm{~B}$ & $.3(4)$ \\
\hline C26A-C27A-C28A & & B-Mo1B & & $-\mathrm{P} 2 \mathrm{~B}$ & $8(3)$ \\
\hline C27A-C28A-C23A & 120. & $\mathrm{C} 22 \mathrm{~B}-1$ & & $-\mathrm{P} 2 \mathrm{~B}$ & 3(3) \\
\hline $4 \mathrm{~A}$ & & $\mathrm{C} 2 \mathrm{~B}-\mathrm{I}$ & & $\mathrm{C} 23 \mathrm{~B}$ & $5(4)$ \\
\hline A-P2A & 118. & C2B-I & & $-\mathrm{C} 24 \mathrm{~B}$ & $119.6(4)$ \\
\hline & $122.9(3)$ & C5B-I & & $26 \mathrm{~B}-\mathrm{C} 25 \mathrm{~B}$ & $120.4(4)$ \\
\hline C29A-C30A-C31A & $121.4(4)$ & $\mathrm{C} 3 \mathrm{~B}-\mathrm{N} 2$ & & C27B-C26B & $119.6(4)$ \\
\hline C32A-C31A-C30A & & C3B-N2B-Mo1B & & C27B-C28B-C23B & $121.4(4)$ \\
\hline C31A-C32A-C33A & ) & C20B- & & $-\mathrm{C} 34 \mathrm{~B}$ & 1(4) \\
\hline C32A-C33A-C34A & $120.1(4)$ & $\mathrm{N} 1 \mathrm{~B}-\mathrm{C}$ & & $\mathrm{C} 30 \mathrm{~B}-($ & $119.7(3)$ \\
\hline C33A-C34A-C29A & $120.7(4)$ & N1B-C2B-C1B & & C34B-C29B-P2B & $121.9(3)$ \\
\hline O1A-C35A-Mo1A & $176.7(3)$ & $\mathrm{C} 3 \mathrm{~B}-\mathrm{C}$ & & C31B-C30B-C29B & $120.7(4)$ \\
\hline O2A-C36A-Mo1A & $175.6(3)$ & $\mathrm{N} 2 \mathrm{~B}-\mathrm{C} 3 \mathrm{~B}-\mathrm{C} 2 \mathrm{~B}$ & & C30B-C31B-C $32 B$ & $120.2(4)$ \\
\hline C36B-Mo1B-C35B & $95.34(16)$ & $\mathrm{N} 2 \mathrm{~B}-\mathrm{C} 3 \mathrm{~B}-\mathrm{C} 4 \mathrm{~B}$ & & C33B-C32B-C31B & $119.7(4)$ \\
\hline C36B-Mo1B-N1B & $161.92(15)$ & $\mathrm{C} 2 \mathrm{~B}-\mathrm{C}$ & & C $32 B-C 33 B-C 34 B$ & $120.4(4)$ \\
\hline C35B-Mo1B-N1B & $96.72(14)$ & & & C33B-C34B-C29B & $120.9(4)$ \\
\hline C36B-Mo1B-N2B & $99.17(14)$ & C5B-C6B-C7B & & O1B-C35B-Mo1B & $175.7(3)$ \\
\hline C35B-Mo1B-N2B & $160.93(14)$ & C6B-C7B-P1B & $113.5(3)$ & O2B-C36B-Mo1B & $174.1(4)$ \\
\hline N1B-Mo1B-N2B & $72.47(12)$ & C13B-C8B-C9B & $118.0(4)$ & & \\
\hline
\end{tabular}




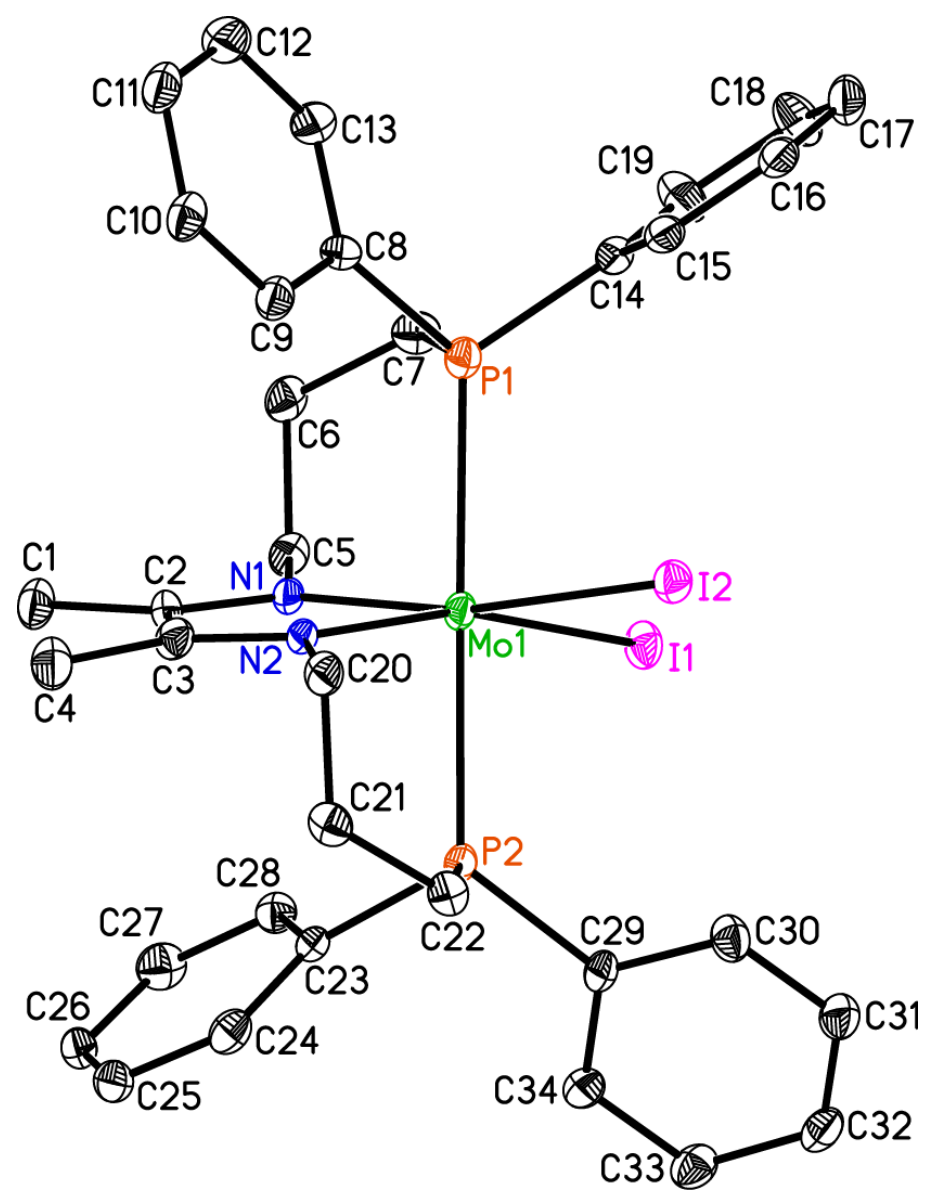

Figure S2. The solid-state structure of $\mathbf{2}$ shown at 30\% probability ellipsoids. Hydrogen atoms are omitted for clarity. 
Table S3. Bond lengths $(\AA)$ and angles $\left({ }^{\circ}\right)$ determined for $\mathbf{2}$.

\begin{tabular}{|c|c|c|c|c|c|}
\hline I1-Mo1 & $2.8261(8)$ & $\mathrm{C} 1-\mathrm{C} 2$ & $528(11)$ & C18-C19 & $0(13)$ \\
\hline I2-Mo1 & $2.8468(8)$ & $\mathrm{C} 2-\mathrm{C} 3$ & $386(11)$ & C20-C21 & $3(11)$ \\
\hline Mo1-N2 & $2.064(6)$ & $\mathrm{C} 3-\mathrm{C} 4$ & $515(11)$ & $\mathrm{C} 21-\mathrm{C} 22$ & $4(11)$ \\
\hline Mo1-N1 & $2.064(6)$ & C5-C6 & $553(11)$ & C23-C28 & $3(11)$ \\
\hline Mo1-P2 & $2.545(2)$ & C6-C7 & $518(11)$ & C23-C24 & $4(11)$ \\
\hline Mo1-P1 & $2.565(2)$ & C8-C9 & $378(11)$ & $\mathrm{C} 24-\mathrm{C} 25$ & $3(11)$ \\
\hline P1-C14 & $1.833(8)$ & C8-C13 & $385(11)$ & $\mathrm{C} 25-\mathrm{C} 26$ & $4(12)$ \\
\hline P1-C8 & $1.841(8)$ & C9-C10 & 379(11) & C26-C27 & $7(12)$ \\
\hline P1-C7 & $1.842(8)$ & C10-C11 & $375(12)$ & C27-C28 & $6(11)$ \\
\hline $\mathrm{P} 2-\mathrm{C} 23$ & $1.828(7)$ & $\mathrm{C} 11-\mathrm{C} 12$ & $373(13)$ & C29-C30 & $7(11)$ \\
\hline P2-C29 & $1.829(7)$ & C12-C13 & $386(13)$ & C29-C34 & $5(11)$ \\
\hline $\mathrm{P} 2-\mathrm{C} 22$ & $1.836(7)$ & C14-C19 & $385(12)$ & C30-C31 & $7(11)$ \\
\hline N1-C2 & $1.366(10)$ & C14-C15 & $388(11)$ & C31-C32 & $2(12)$ \\
\hline $\mathrm{N} 1-\mathrm{C} 5$ & $1.478(10)$ & C15-C16 & $386(11)$ & C32-C33 & $9(12)$ \\
\hline N2-C3 & $1.362(9)$ & C16-C17 & $386(13)$ & C33-C34 & $7(11)$ \\
\hline $\mathrm{N} 2-\mathrm{C} 20$ & $1.483(10)$ & C17-C18 & \multicolumn{3}{|c|}{$1.373(14)$} \\
\hline \multicolumn{2}{|l|}{ N2-Mo1-N1 } & C22-P2-Mo1 & $108.7(3)$ & C15-C14-P1 & $119.0(6)$ \\
\hline N2-Mo1-P2 & $77.09(16)$ & C2-N1-C5 & $118.2(6)$ & C16-C15-C14 & $121.6(8)$ \\
\hline N1-Mo1-P2 & $99.36(18)$ & C2-N1-Mo1 & $118.1(5)$ & C15-C16-C17 & $120.4(9)$ \\
\hline N2-Mo1-P1 & $104.66(17)$ & C5-N1-Mo1 & $123.4(5)$ & C18-C17-C16 & $118.6(8)$ \\
\hline N1-Mo1-P1 & $80.28(18)$ & C3-N2-C20 & $118.4(6)$ & C17-C18-C19 & 121.1(9) \\
\hline P2-Mo1-P1 & $178.01(7)$ & C3-N2-Mo1 & $118.8(5)$ & C14-C19-C18 & $120.6(9)$ \\
\hline N2-Mo1-I1 & $163.15(16)$ & C20-N2-Mo1 & $122.1(4)$ & N2-C20-C21 & $112.0(6)$ \\
\hline N1-Mo1-I1 & $97.09(18)$ & N1-C2-C3 & $114.7(6)$ & C20-C21-C22 & $114.8(6)$ \\
\hline P2-Mo1-I1 & $90.10(5)$ & $\mathrm{N} 1-\mathrm{C} 2-\mathrm{C} 1$ & $120.7(7)$ & C21-C22-P2 & $115.7(5)$ \\
\hline P1-Mo1-I1 & $88.00(5)$ & $\mathrm{C} 3-\mathrm{C} 2-\mathrm{C} 1$ & $124.5(7)$ & C28-C23-C24 & $118.4(7)$ \\
\hline N2-Mo1-I2 & $99.00(17)$ & $\mathrm{N} 2-\mathrm{C} 3-\mathrm{C} 2$ & $113.6(6)$ & C28-C23-P2 & $119.2(6)$ \\
\hline N1-Mo1-I2 & $167.40(17)$ & $\mathrm{N} 2-\mathrm{C} 3-\mathrm{C} 4$ & $123.4(7)$ & C24-C23-P2 & $122.3(6)$ \\
\hline P2-Mo1-I2 & $89.59(5)$ & $\mathrm{C} 2-\mathrm{C} 3-\mathrm{C} 4$ & $123.0(7)$ & C25-C24-C23 & 119.1(8) \\
\hline P1-Mo1-I2 & $91.07(5)$ & $\mathrm{N} 1-\mathrm{C} 5-\mathrm{C} 6$ & $111.0(6)$ & $\mathrm{C} 26-\mathrm{C} 25-\mathrm{C} 24$ & $121.4(8)$ \\
\hline I1-Mo1-I2 & $91.71(3)$ & C7-C6-C5 & $113.2(6)$ & C25-C26-C27 & $120.2(8)$ \\
\hline C14-P1-C8 & $99.8(3)$ & C6-C7-P1 & $115.7(6)$ & C26-C27-C28 & $119.4(8)$ \\
\hline C14-P1-C7 & $104.0(4)$ & C9-C8-C13 & $117.8(7)$ & $\mathrm{C} 27-\mathrm{C} 28-\mathrm{C} 23$ & $121.4(7)$ \\
\hline C8-P1-C7 & $103.0(4)$ & C9-C8-P1 & $121.6(6)$ & С30-C29-C34 & $117.7(7)$ \\
\hline C14-P1-Mo & $122.2(3)$ & C13-C8-P1 & $120.5(6)$ & C30-C29-P2 & $119.6(6)$ \\
\hline C8-P1-Mo1 & $117.2(3)$ & C8-C9-C10 & $121.0(8)$ & C34-C29-P2 & $122.7(6)$ \\
\hline C7-P1-Mo1 & $108.4(3)$ & C11-C10-C9 & $120.6(8)$ & C31-C30-C29 & $121.9(8)$ \\
\hline C23-P2-C29 & $104.4(3)$ & $\mathrm{C} 12-\mathrm{C} 11-\mathrm{C} 10$ & 119.3(8) & C32-C31-C30 & $119.9(8)$ \\
\hline C23-P2-C22 & $105.6(3)$ & C11-C12-C13 & $119.8(9)$ & C31-C32-C33 & $120.1(8)$ \\
\hline C29-P2-C22 & $102.5(3)$ & C8-C13-C12 & $121.4(8)$ & C34-C33-C32 & $120.2(8)$ \\
\hline C23-P2-Mo & $111.6(2)$ & C19-C14-C15 & $117.8(8)$ & C33-C34-C29 & $120.1(7)$ \\
\hline C29-P2-Mo & $122.6(2)$ & C19-C14-P1 & $123.2(6)$ & & \\
\hline
\end{tabular}




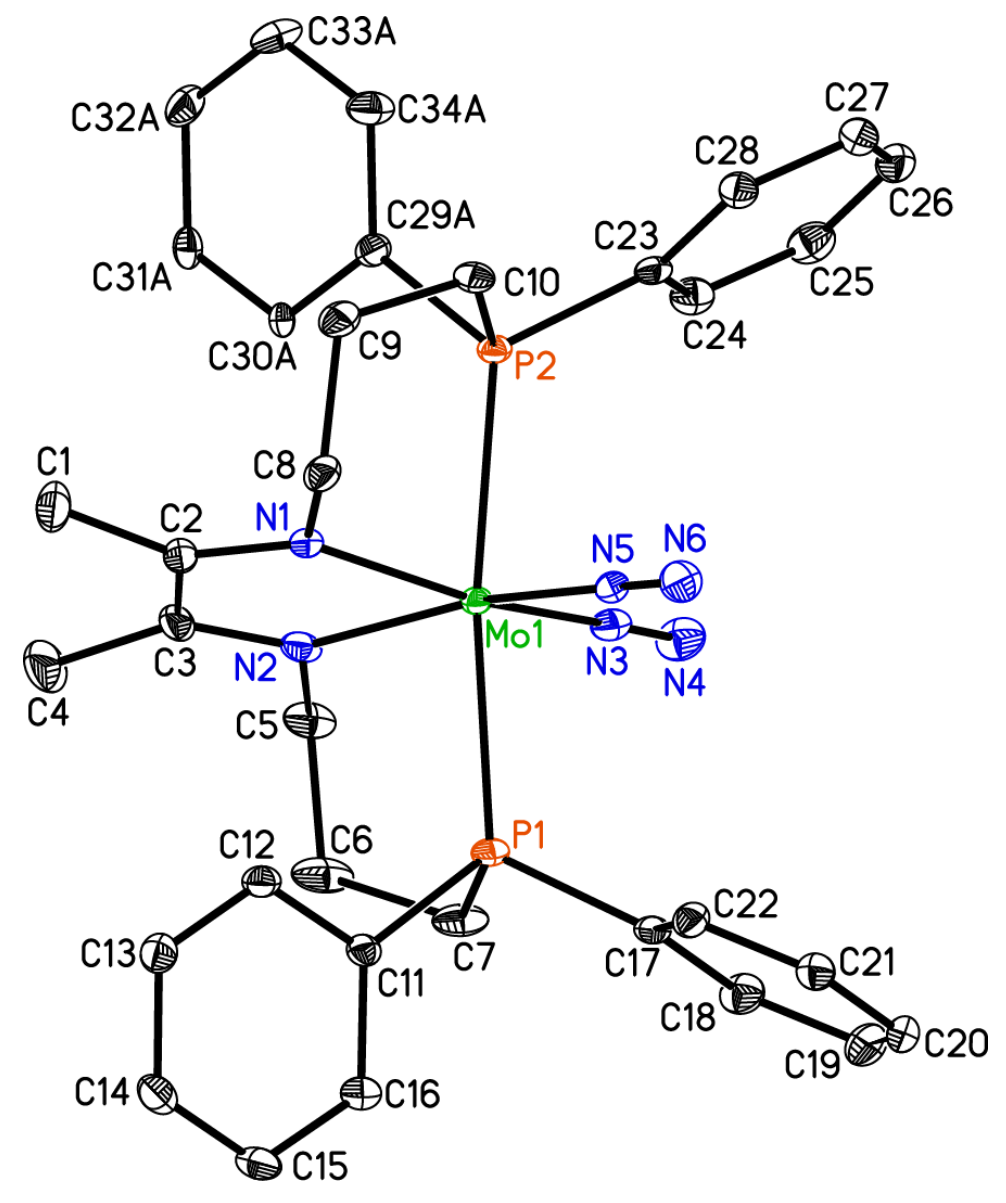

Figure S3. The molecular structure of $\mathbf{3}$ displayed at 30\% probability ellipsoids. Hydrogen atoms and two co-crystallized THF molecules omitted for clarity. The phenyl ring containing C29AC34A was found to exhibit positional disorder and C29B-C34B have been omitted for clarity. 
Table S4. Bond lengths $(\AA)$ and angles $\left({ }^{\circ}\right)$ determined for 3 .

\begin{tabular}{|c|c|c|c|c|c|}
\hline Mo1-N3 & $2.002(2)$ & $\mathrm{N} 2-\mathrm{C} 3$ & $1.347(3)$ & C14-C15 & $1.381(4)$ \\
\hline Mo1-N5 & $2.003(2)$ & $\mathrm{N} 2-\mathrm{C} 5$ & $1.472(3)$ & C15-C16 & $1.379(4)$ \\
\hline Mo1-N2 & $2.101(2)$ & N3-N4 & $1.134(3)$ & $\mathrm{C} 17-\mathrm{C} 22$ & $1.395(4)$ \\
\hline Mo1-N1 & $2.103(2)$ & N5-N6 & $1.128(3)$ & C17-C18 & $1.396(3)$ \\
\hline Mo1-P1 & $2.4659(6)$ & $\mathrm{C} 1-\mathrm{C} 2$ & $1.511(4)$ & C18-C19 & $1.387(4)$ \\
\hline Mo1-P2 & $2.4670(6)$ & $\mathrm{C} 2-\mathrm{C} 3$ & $1.405(4)$ & C19-C20 & $1.375(4)$ \\
\hline P1-C17 & $1.827(3)$ & $\mathrm{C} 3-\mathrm{C} 4$ & $1.513(4)$ & C20-C21 & $1.384(4)$ \\
\hline $\mathrm{P} 1-\mathrm{C} 11$ & $1.830(3)$ & C5-C6 & $1.532(4)$ & $\mathrm{C} 21-\mathrm{C} 22$ & $1.391(4)$ \\
\hline P1-C7 & $1.838(2)$ & C6-C7 & $1.530(4)$ & C23-C28 & (3) \\
\hline $\mathrm{P} 2-\mathrm{C} 23$ & $(2)$ & C8-C9 & (3) & C23-C24 & (4) \\
\hline $\mathrm{P} 2-\mathrm{C} 10$ & (2) & C9-C10 & (3) & C24-C25 & (4) \\
\hline P2-C29B & (6) & C11-C12 & (3) & C25-C26 & (4) \\
\hline P2-C29A & (6) & C11-C16 & (3) & C26-C27 & (4) \\
\hline $\mathrm{N} 1-\mathrm{C} 2$ & (3) & C12-C13 & (4) & $\mathrm{C} 27-\mathrm{C} 28$ & (4) \\
\hline N1-C8 & (3) & $\mathrm{C} 13-\mathrm{C} 14$ & (4) & & \\
\hline N3-Mo1-N5 & $97.29(8)$ & C23-P2-Mo1 & $119.20(8)$ & C16-C11-P1 & $121.95(19)$ \\
\hline N3-Mo1-N2 & $96.32(8)$ & C10-P2-Mo1 & $110.65(8)$ & C13-C12-C11 & $120.7(2)$ \\
\hline N5-Mo1-N2 & $162.81(8)$ & C29B-P2-Mo1 & $115.7(2)$ & C14-C13-C12 & $120.1(2)$ \\
\hline N3-Mo1-N1 & $164.08(8)$ & C29A-P2-Mo1 & 119.1(2) & C15-C14-C13 & $119.5(2)$ \\
\hline N5-Mo1-N1 & $95.39(8)$ & C2-N1-C8 & $118.5(2)$ & $\mathrm{C} 16-\mathrm{C} 15-\mathrm{C} 14$ & $120.9(2)$ \\
\hline N2-Mo1-N1 & $73.24(8)$ & C2-N1-Mo1 & $119.24(18)$ & C15-C16-C11 & $120.3(2)$ \\
\hline N3-Mo1-P1 & $81.69(6)$ & C8-N1-Mo1 & $121.81(15)$ & C22-C17-C18 & $118.2(2)$ \\
\hline N5-Mo1-P1 & $91.05(6)$ & C3-N2-C5 & $118.8(2)$ & $\mathrm{C} 22-\mathrm{C} 17-\mathrm{P} 1$ & $117.81(18)$ \\
\hline N2-Mo1-P1 & $80.59(6)$ & C3-N2-Mo1 & 118.91(17) & C18-C17-P1 & $123.9(2)$ \\
\hline N1-Mo1-P1 & $107.70(6)$ & C5-N2-Mo1 & 121.91(17) & C19-C18-C17 & $120.0(3)$ \\
\hline N3-Mo1-P2 & $93.12(6)$ & N4-N3-Mo1 & $175.8(2)$ & C20-C19-C18 & $121.2(3)$ \\
\hline N5-Mo1-P2 & $84.40(6)$ & N6-N5-Mo1 & $174.9(2)$ & C19-C20-C21 & $119.8(3)$ \\
\hline N2-Mo1-P2 & $105.29(6)$ & N1-C2-C3 & $113.9(2)$ & C20-C21-C22 & $119.4(3)$ \\
\hline N1-Mo1-P2 & $78.57(5)$ & $\mathrm{N} 1-\mathrm{C} 2-\mathrm{C} 1$ & $122.9(3)$ & C21-C22-C17 & $121.4(2)$ \\
\hline P1-Mo1-P2 & $172.63(2)$ & C3-C2-C1 & $123.1(2)$ & C28-C23-C24 & $118.3(2)$ \\
\hline C17-P1-C11 & $103.16(11)$ & $\mathrm{N} 2-\mathrm{C} 3-\mathrm{C} 2$ & $114.2(2)$ & C28-C23-P2 & $124.34(19)$ \\
\hline C17-P1-C7 & $103.69(12)$ & $\mathrm{N} 2-\mathrm{C} 3-\mathrm{C} 4$ & $122.7(3)$ & C24-C23-P2 & $117.37(19)$ \\
\hline C11-P1-C7 & $100.29(12)$ & $\mathrm{C} 2-\mathrm{C} 3-\mathrm{C} 4$ & $123.0(3)$ & $\mathrm{C} 25-\mathrm{C} 24-\mathrm{C} 23$ & $121.3(3)$ \\
\hline C17-P1-Mo1 & $117.90(8)$ & N2-C5-C6 & $112.4(2)$ & C26-C25-C24 & $120.0(3)$ \\
\hline C11-P1-Mo1 & $119.52(8)$ & C7-C6-C5 & $113.7(2)$ & C27-C26-C25 & $119.5(3)$ \\
\hline C7-P1-Mo1 & $109.85(9)$ & C6-C7-P1 & $112.49(19)$ & $\mathrm{C} 26-\mathrm{C} 27-\mathrm{C} 28$ & $120.8(3)$ \\
\hline C23-P2-C10 & $103.16(11)$ & N1-C8-C9 & $112.5(2)$ & $\mathrm{C} 23-\mathrm{C} 28-\mathrm{C} 27$ & $120.1(2)$ \\
\hline C23-P2-C29 & $104.8(2)$ & C8-C9-C10 & $113.6(2)$ & C30A-C29A-P2 & 119.1(4) \\
\hline C10-P2-C29 & 101.1(3) & C9-C10-P2 & $113.42(17)$ & $\mathrm{C} 34 \mathrm{~A}-\mathrm{C} 29 \mathrm{~A}-\mathrm{P} 2$ & $120.9(5)$ \\
\hline C23-P2-C29 & $98.2(2)$ & C12-C11-C16 & $118.5(2)$ & C30B-C29B-P2 & $117.8(5)$ \\
\hline C10-P2-C29 & $104.4(3)$ & C12-C11-P1 & $119.42(18)$ & C34B-C29B-P2 & $122.1(5)$ \\
\hline
\end{tabular}




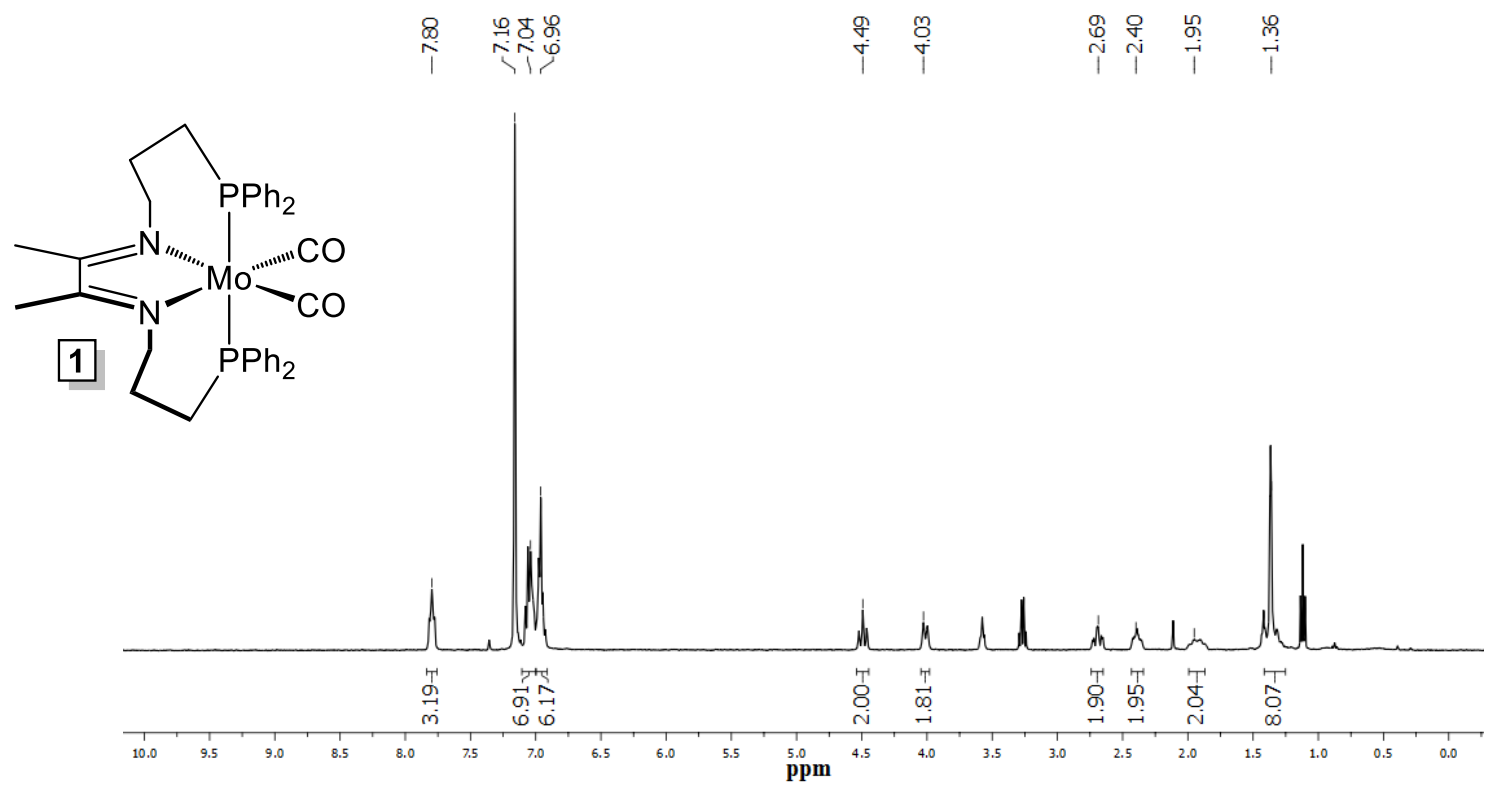

Figure S4. ${ }^{1} \mathrm{H}$ NMR spectrum of 1 in benzene- $d_{6}$ at $25^{\circ} \mathrm{C}$.

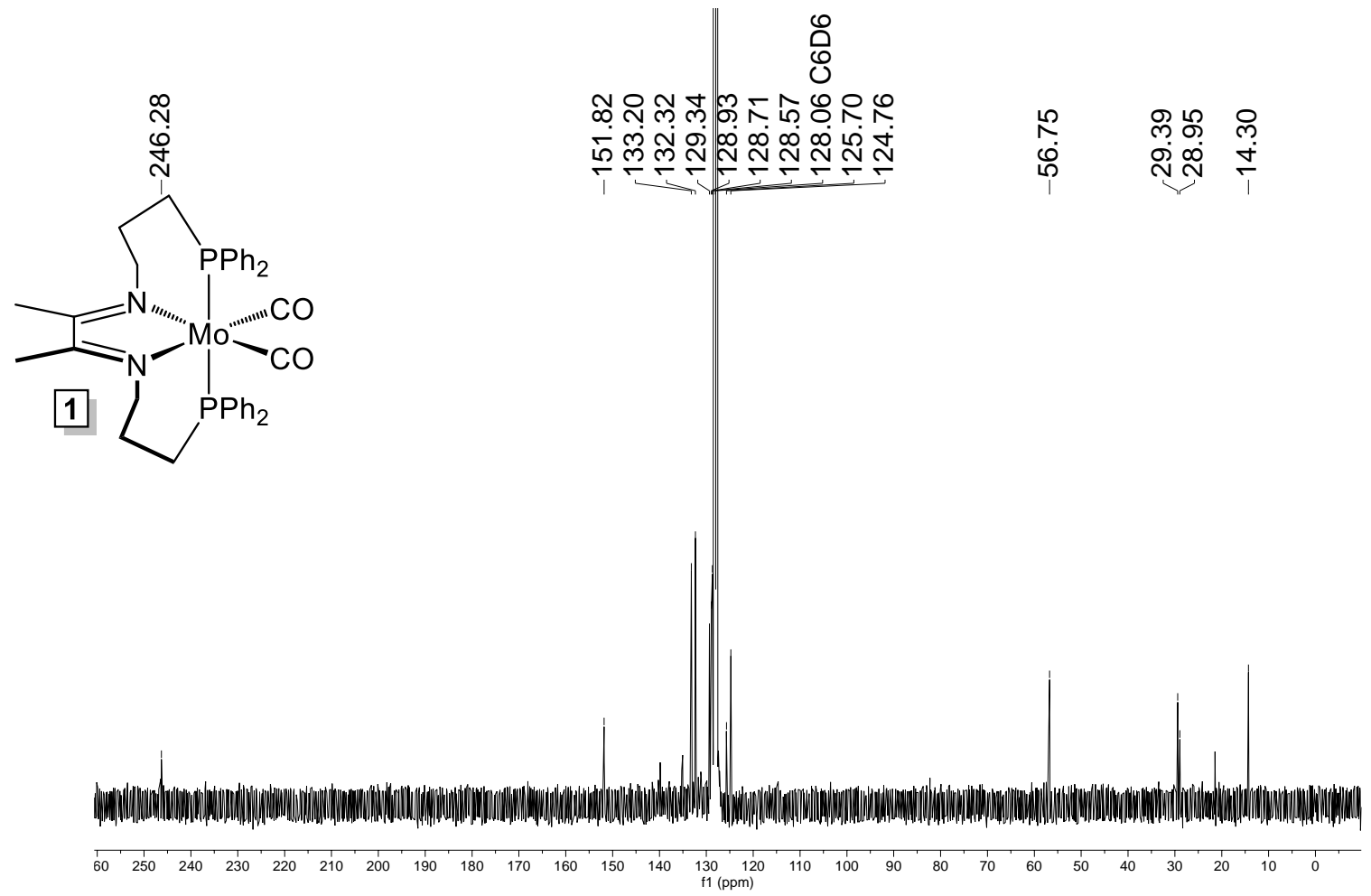

Figure S5. ${ }^{13} \mathrm{C}$ NMR spectrum of $\mathbf{1}$ in benzene- $d_{6}$ at $25{ }^{\circ} \mathrm{C}$. 

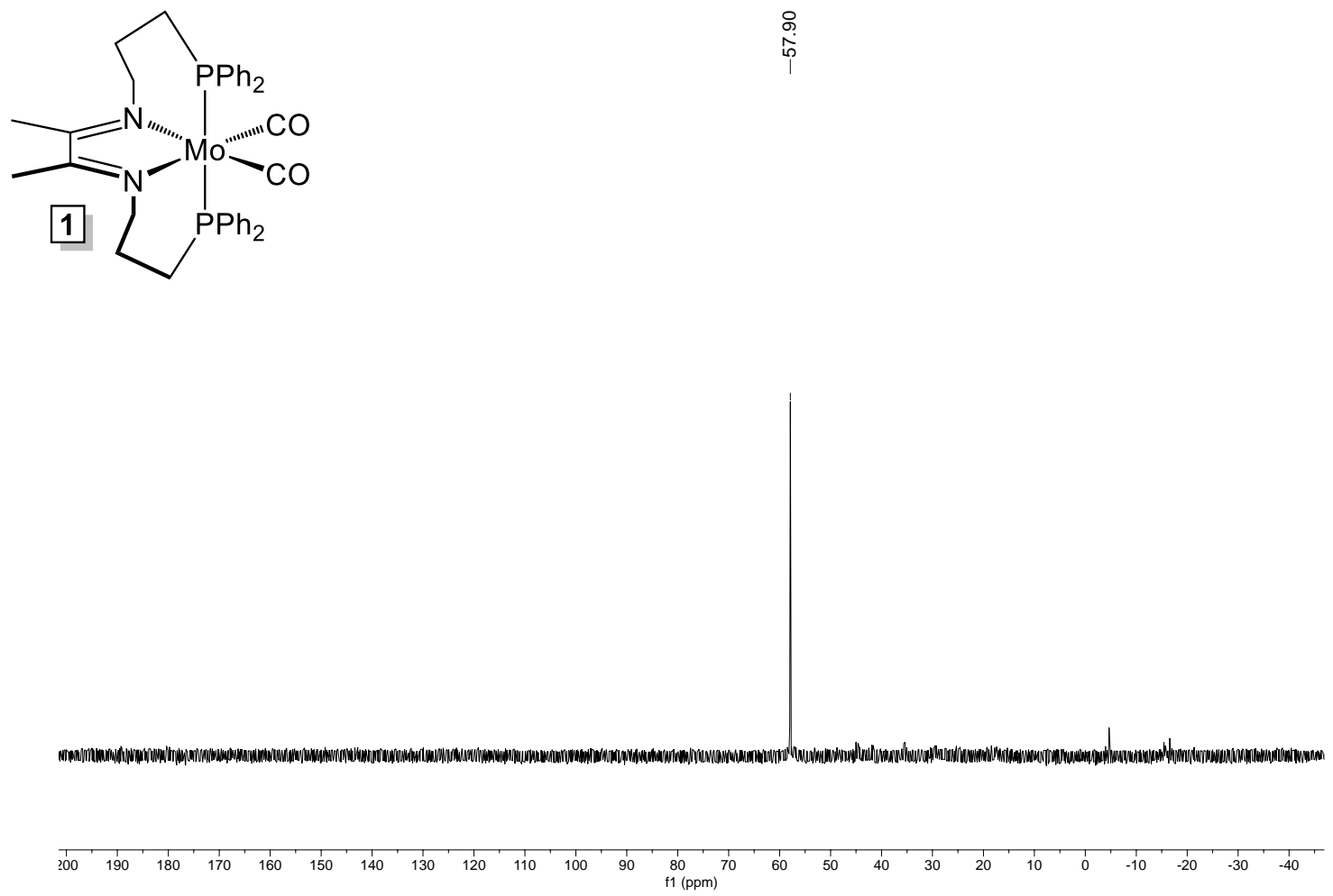

Figure S6. ${ }^{31} \mathrm{P}$ NMR spectrum of $\mathbf{1}$ in benzene- $d_{6}$.

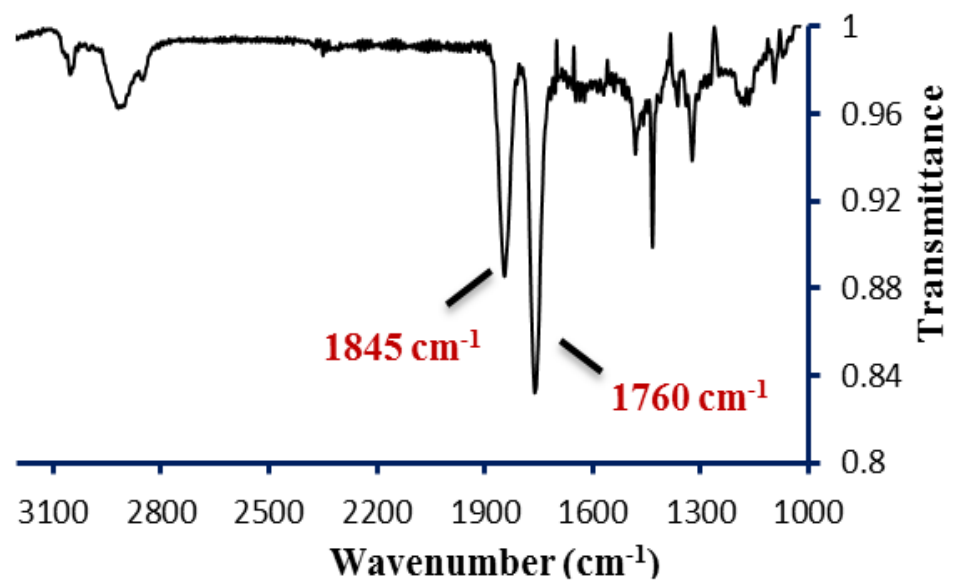

Figure S7. Infrared spectrum of $\mathbf{1}$ in $\mathrm{KBr}$. 


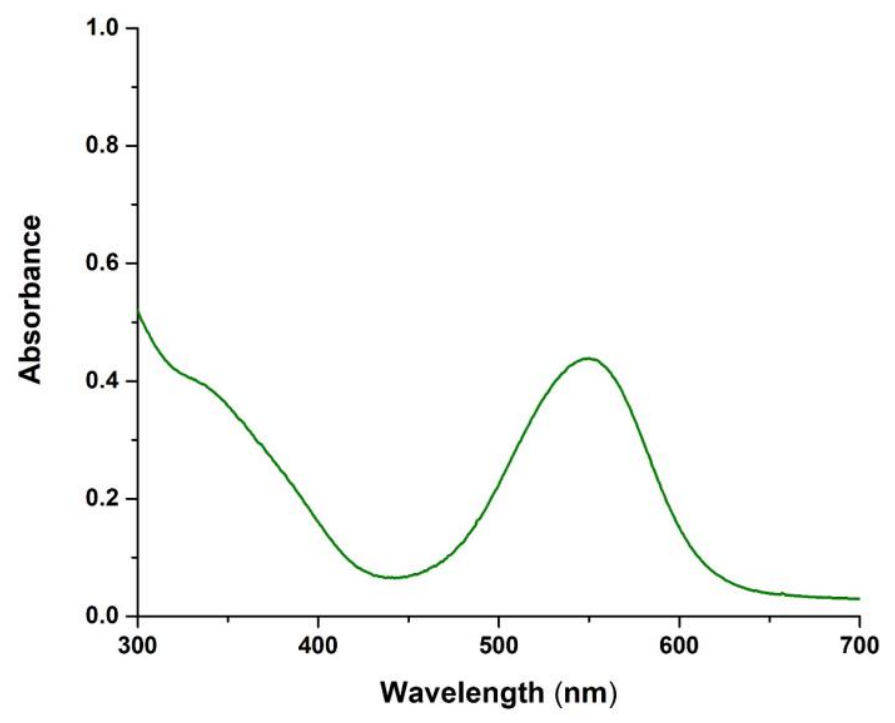

Figure S8. UV-visible spectrum of $0.29 \mathrm{mM} 1$ in toluene.

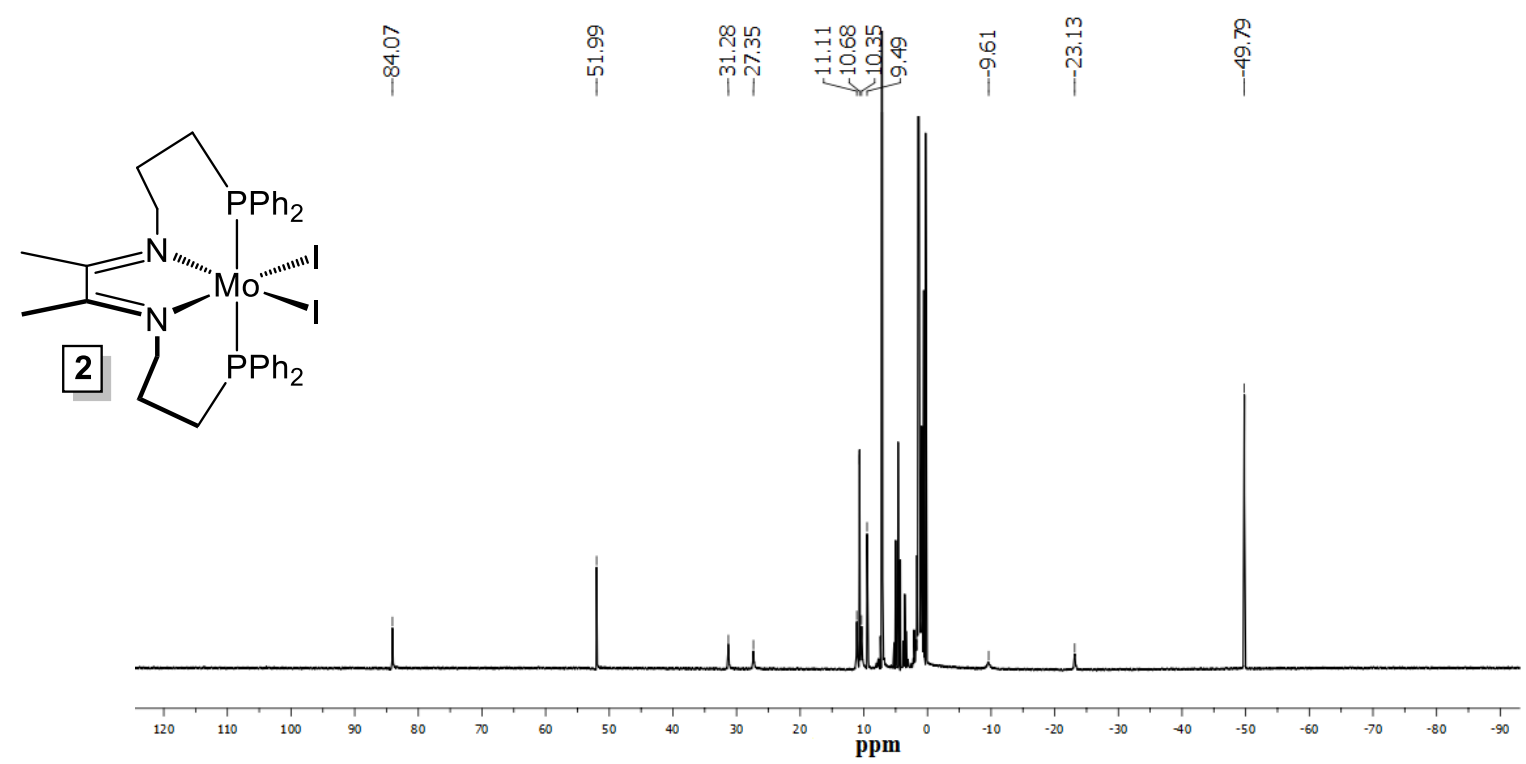

Figure S9. ${ }^{1} \mathrm{H}$ NMR spectrum of 2 in benzene- $d_{6}$ at $25^{\circ} \mathrm{C}$. 


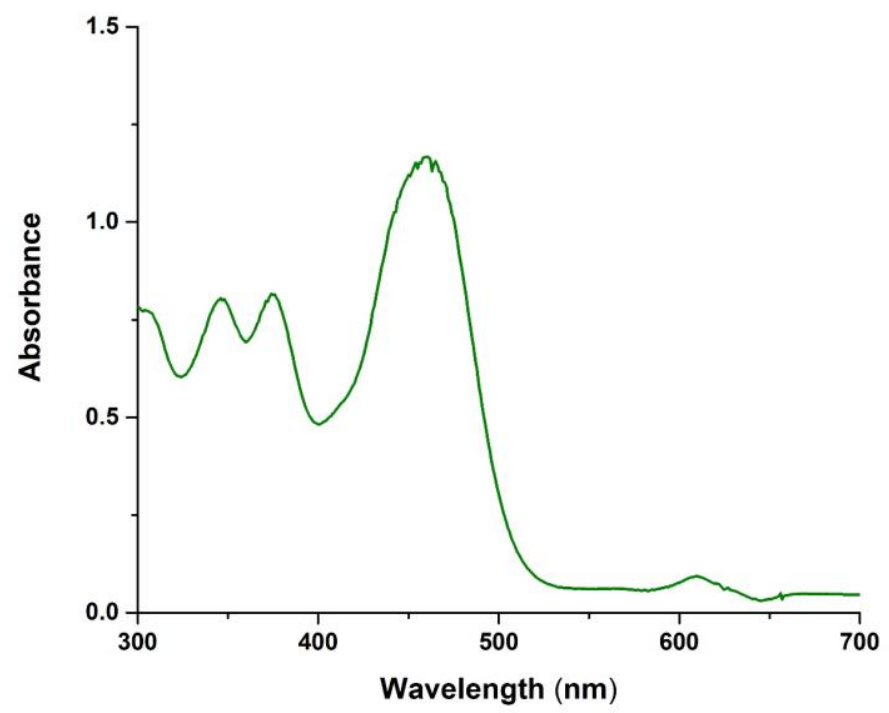

Figure S10. UV-visible spectrum of $0.24 \mathrm{mM} 2$ in THF.

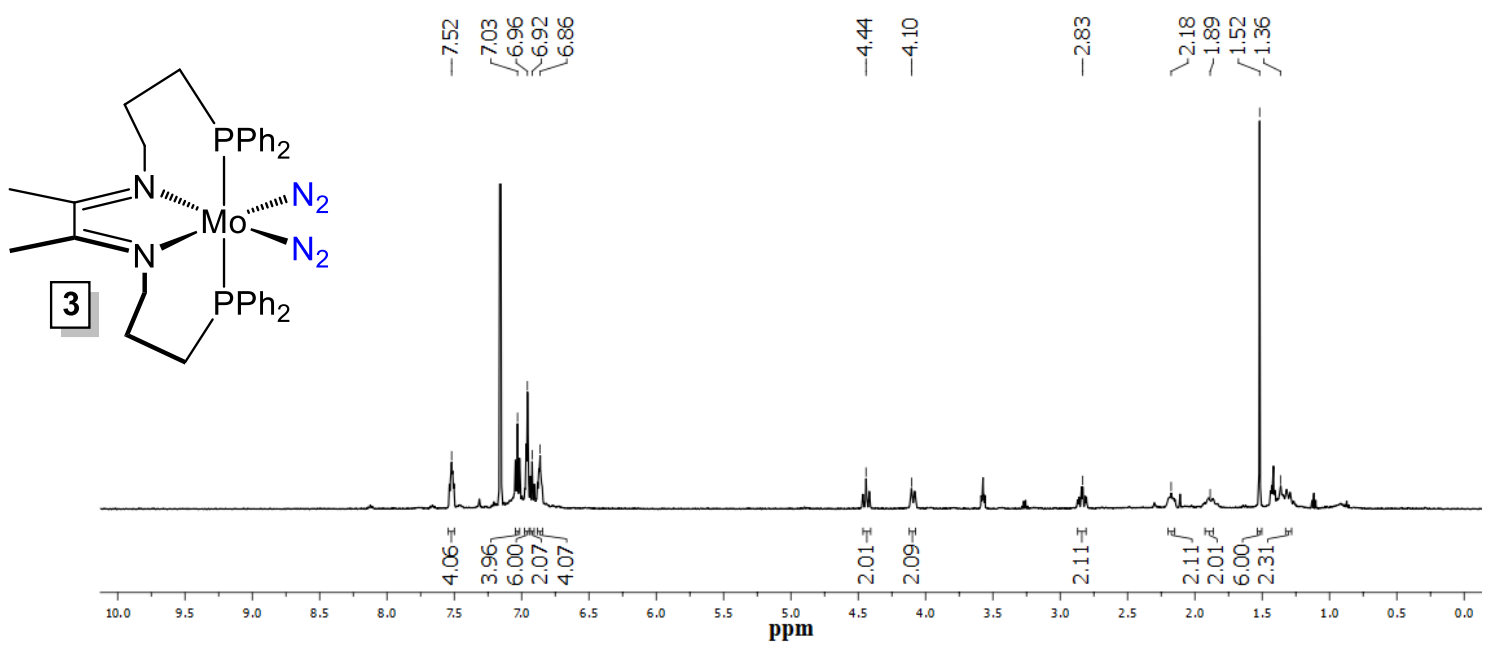

Figure S11. ${ }^{1} \mathrm{H}$ NMR spectrum of $\mathbf{3}$ in benzene- $d_{6}$. 


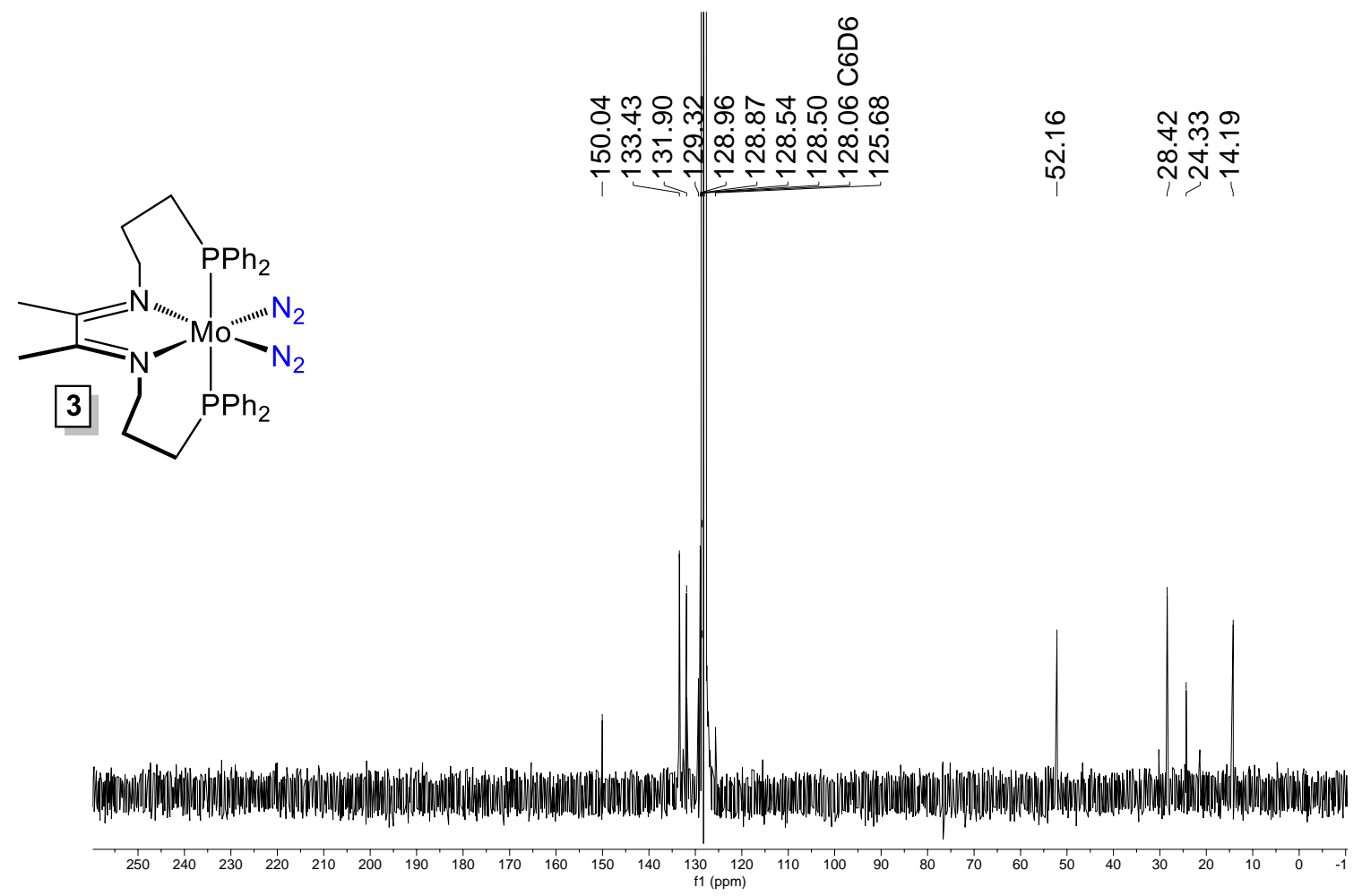

Figure S12. ${ }^{13} \mathrm{C}$ NMR spectrum of 3 in benzene- $d_{6}$.

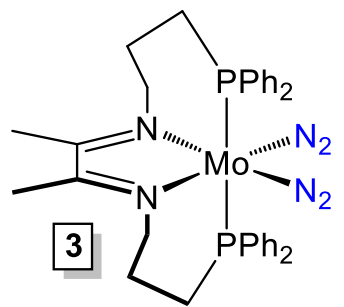

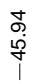

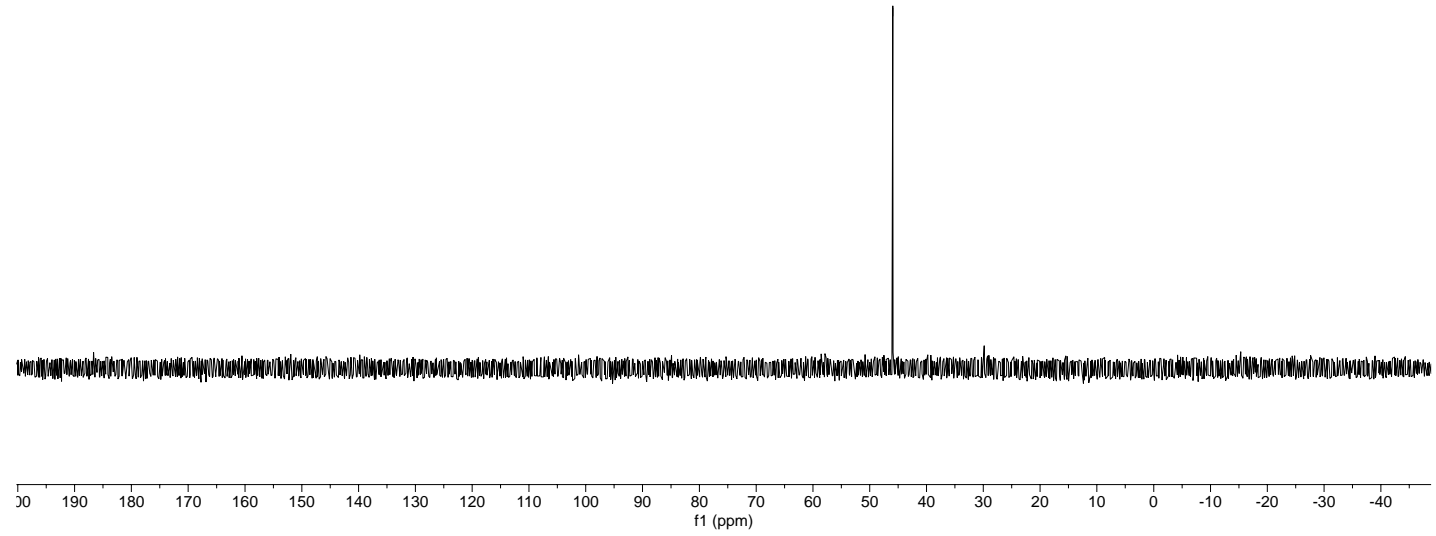

Figure S13. ${ }^{31} \mathrm{P}$ NMR spectrum of 3 in benzene- $d_{6}$. 


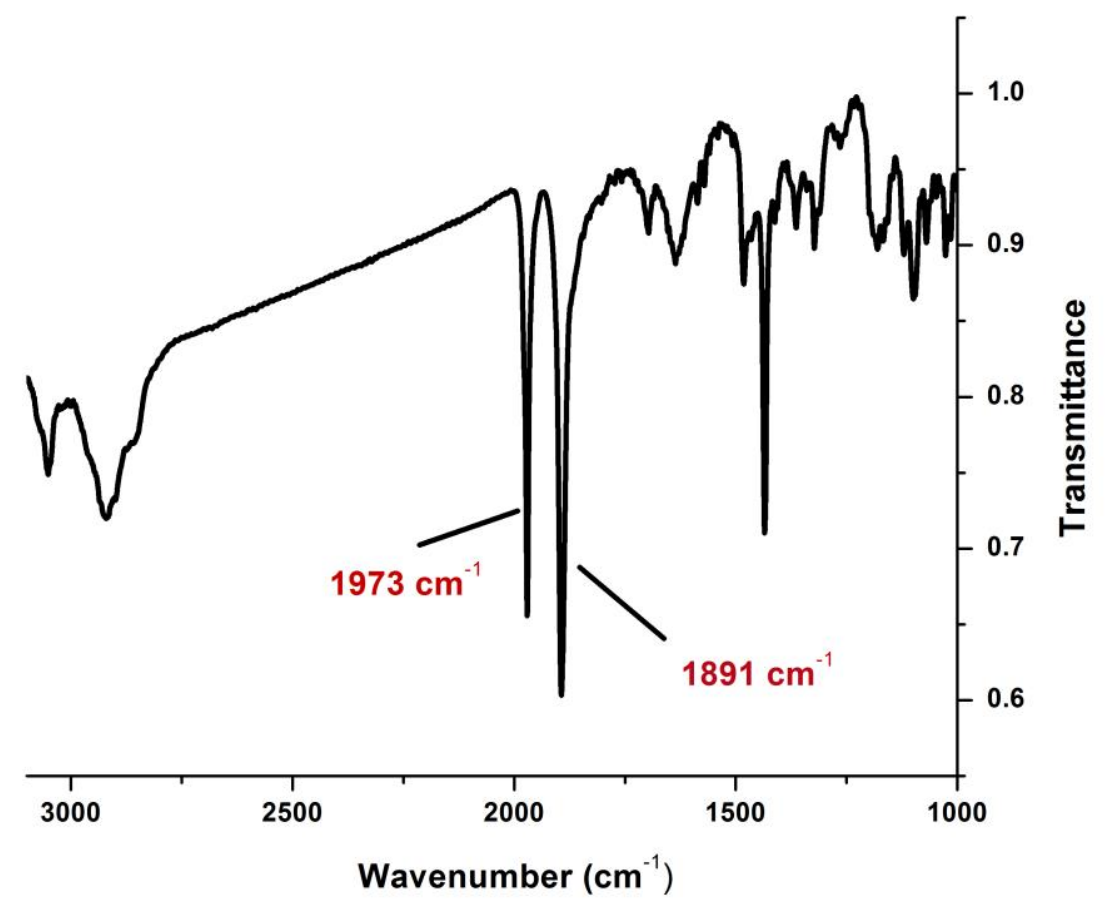

Figure S14. Infrared spectrum of 3 in $\mathrm{KBr}$.

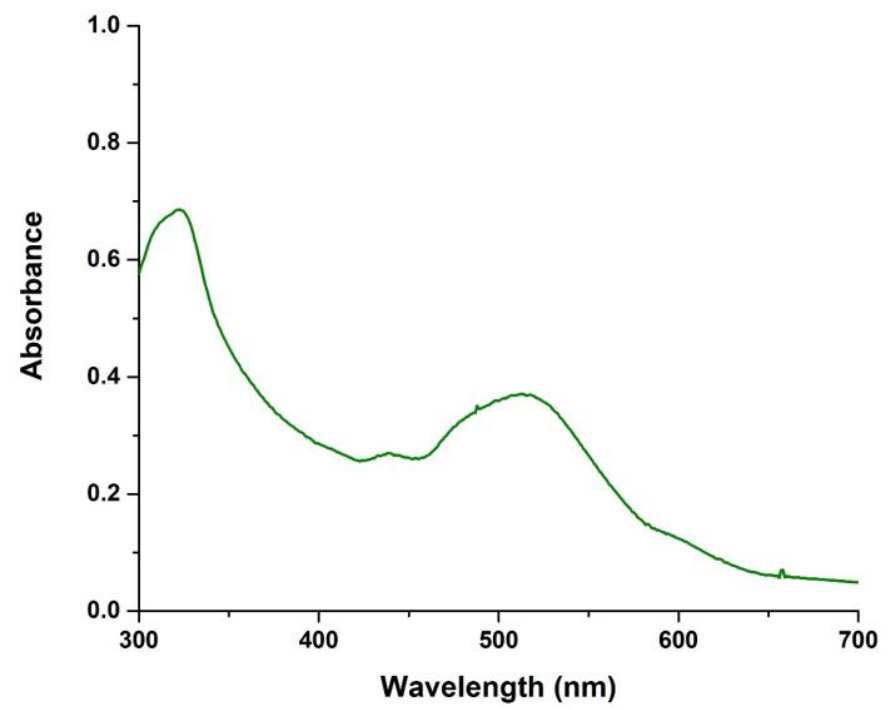

Figure S15. UV-visible spectrum of $0.51 \mathrm{mM} 3$ in THF. 


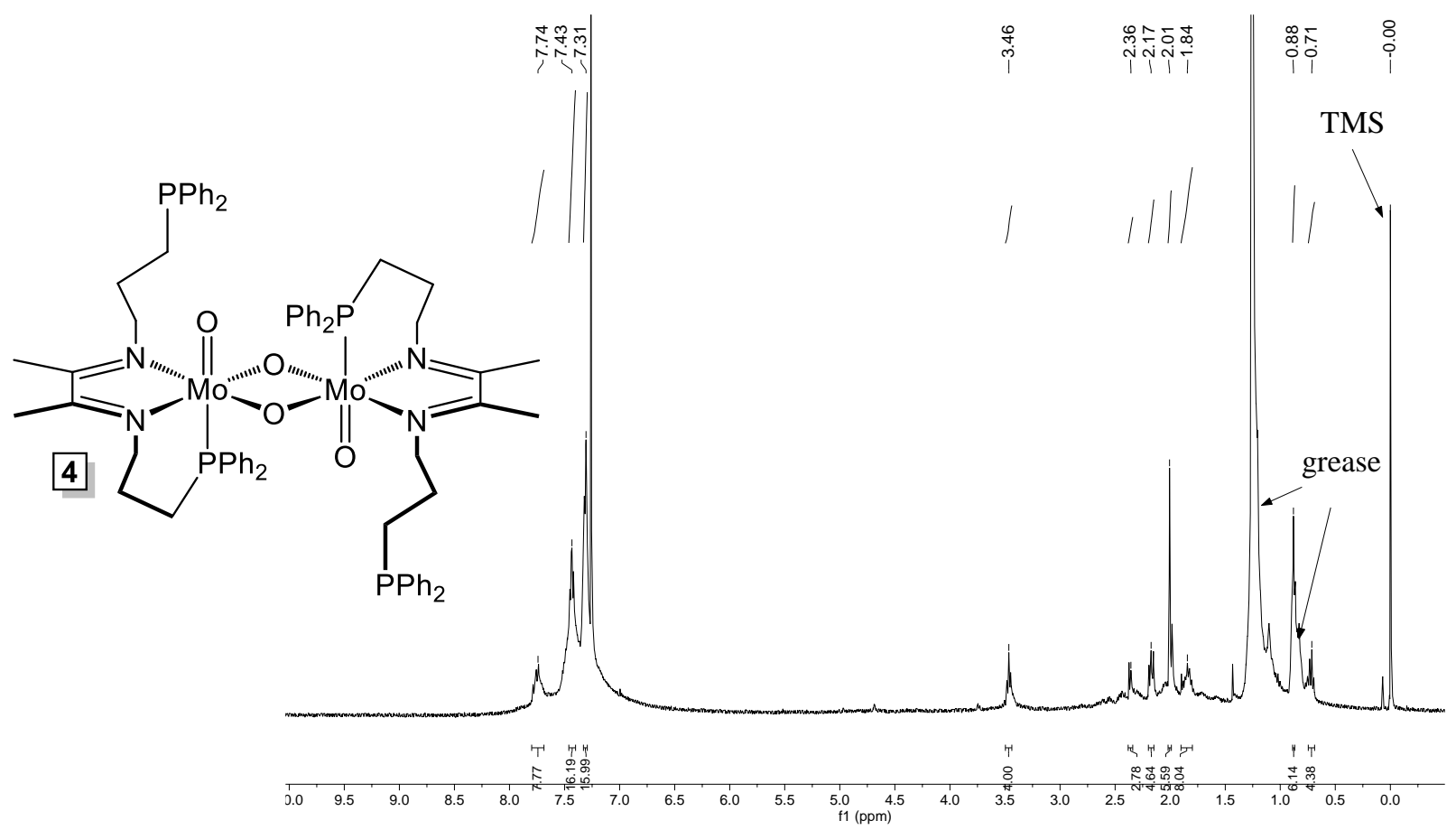

Figure S16. ${ }^{1} \mathrm{H}$ NMR spectrum of 4 in chloroform- $d$.

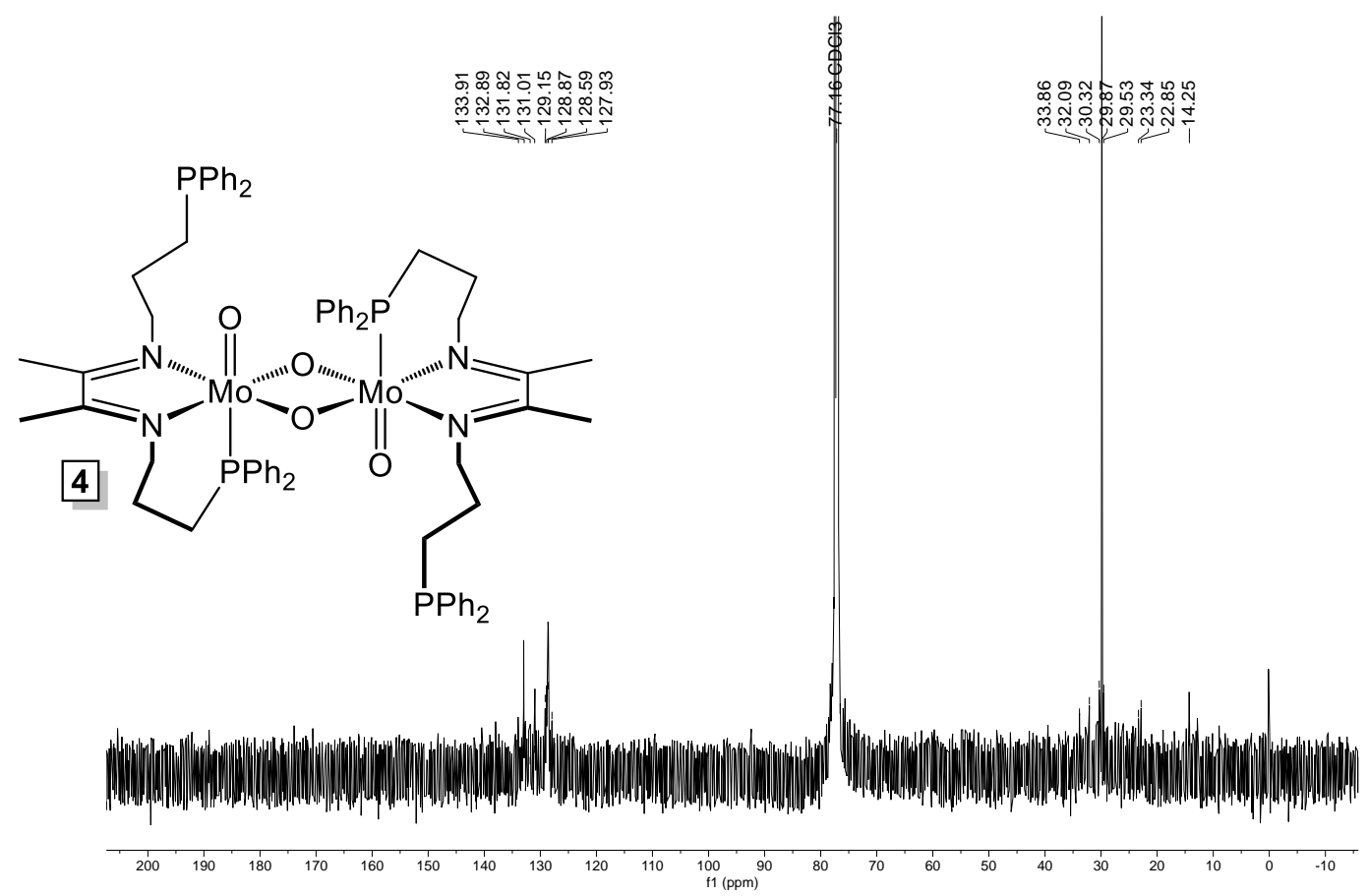

Figure S17. ${ }^{13} \mathrm{C}$ NMR spectrum of $\mathbf{4}$ in chloroform- $d$. 


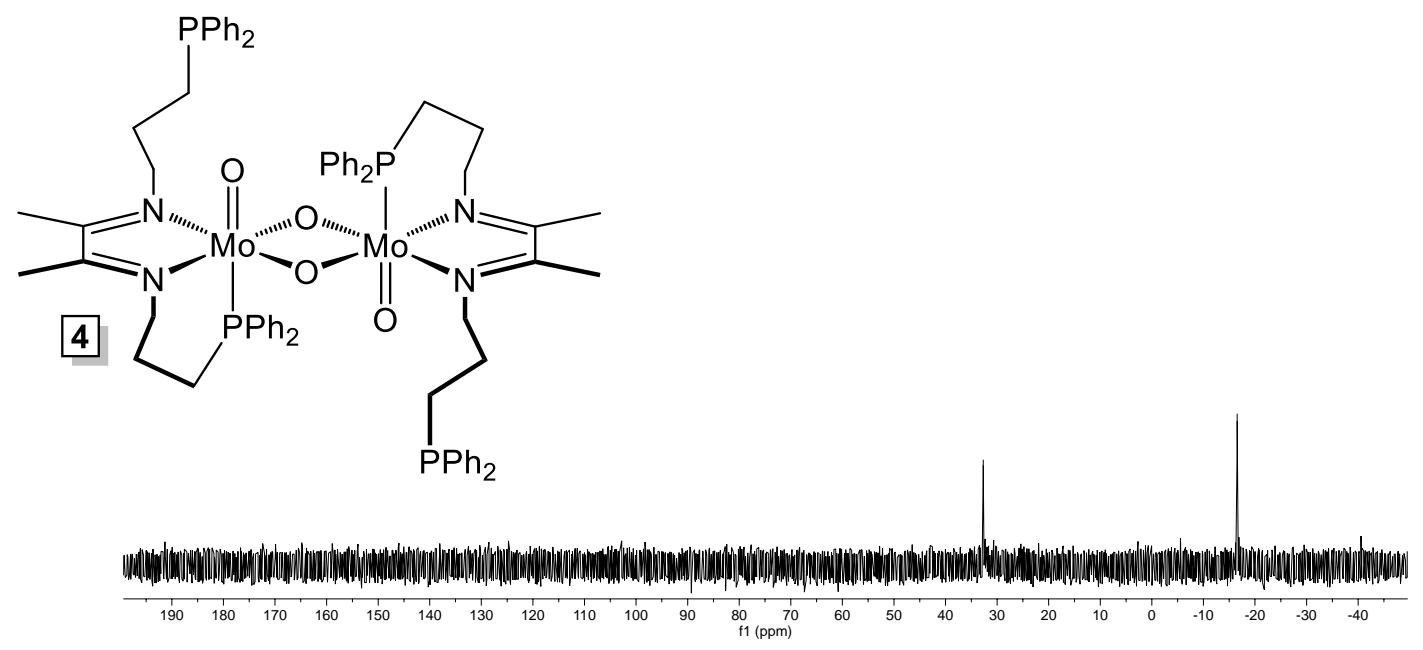

Figure S18. ${ }^{31} \mathrm{P}$ NMR spectrum of 4 in chloroform- $d$.

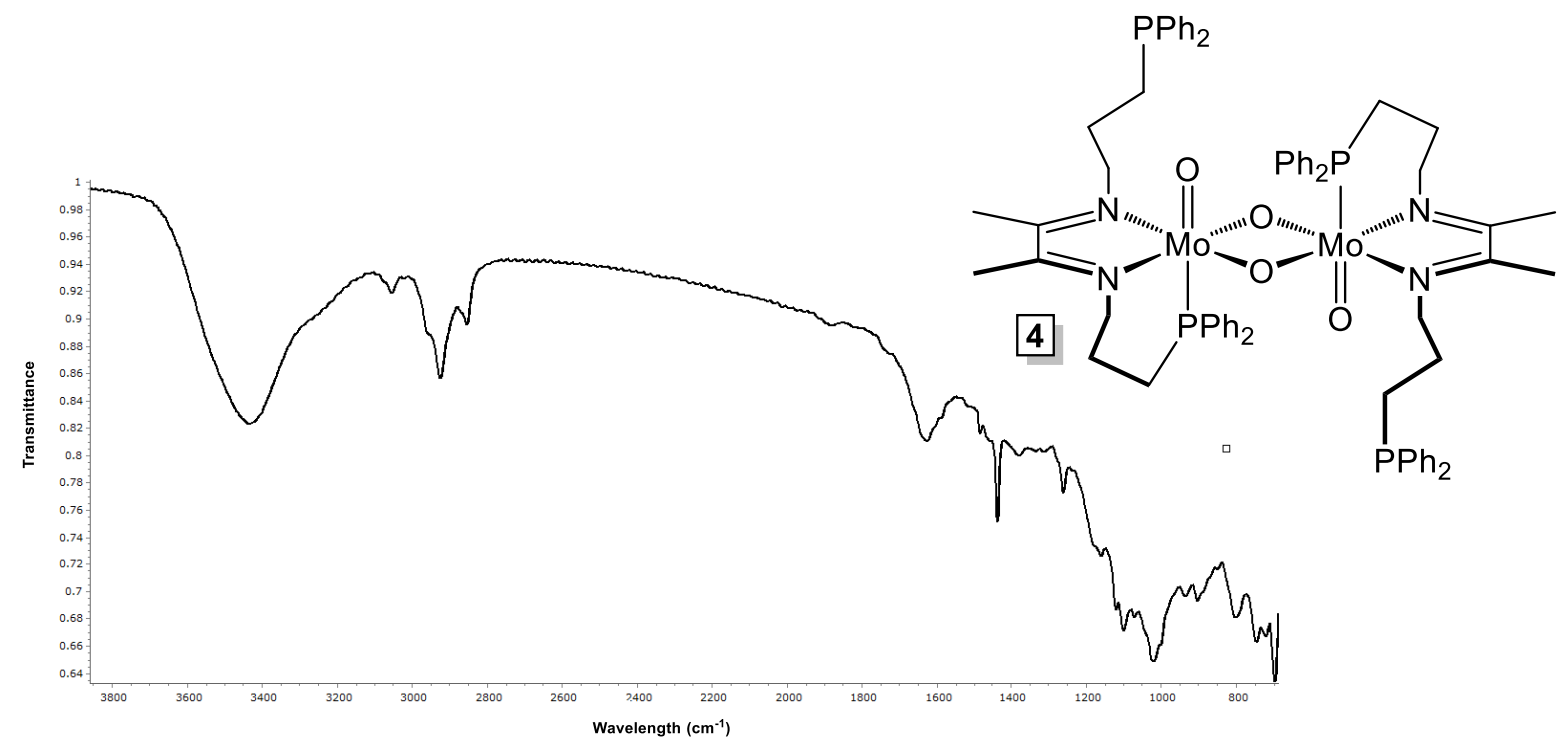

Figure S19. Infrared spectrum of 4 in $\mathrm{KBr}$. 


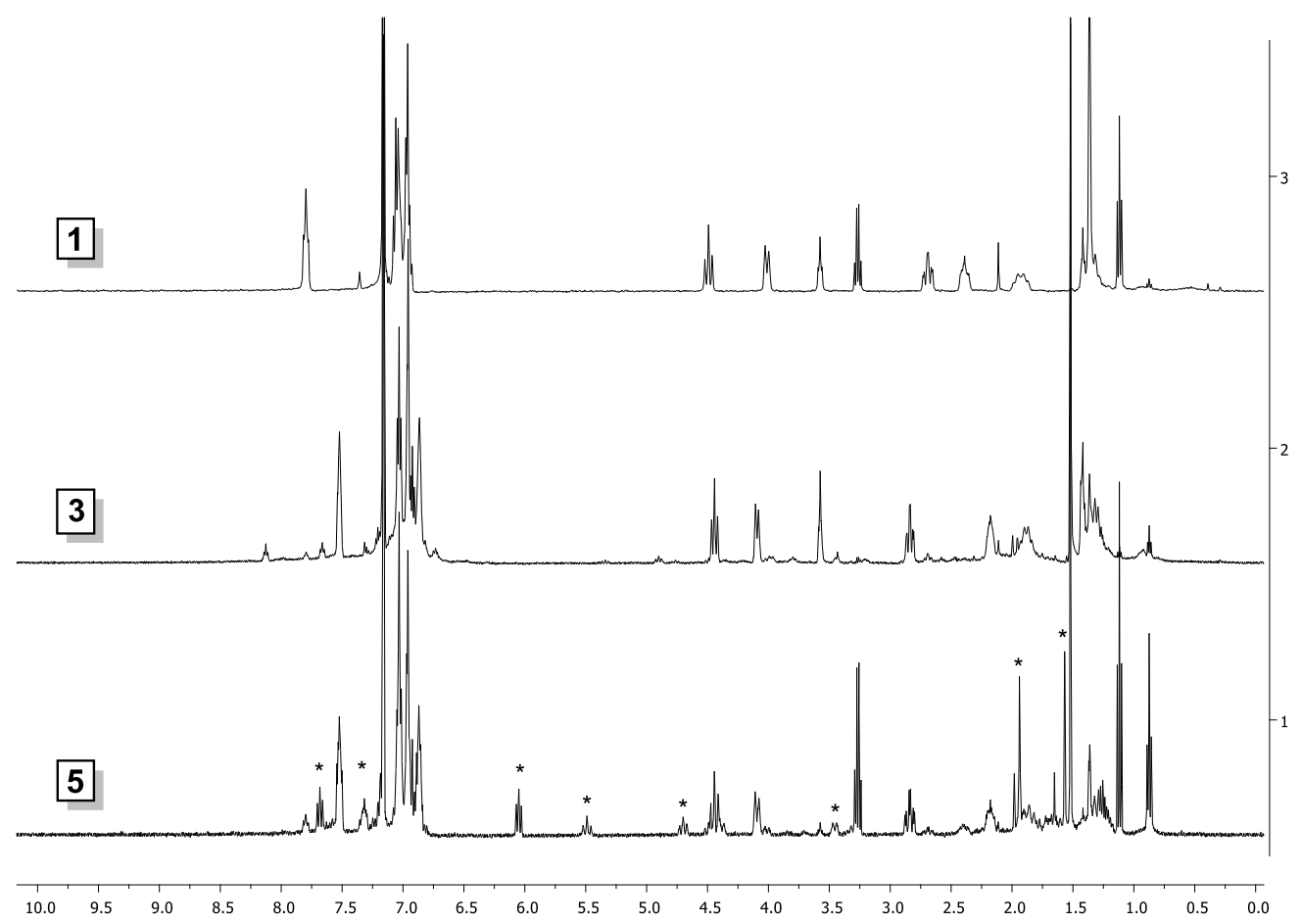

Figure S20. Observation of $\left({ }^{\mathrm{Ph} 2 \mathrm{PP}} \mathrm{DI}\right) \mathrm{Mo}\left(\mathrm{CO}_{3}\right)(\mathrm{CO})(\mathbf{5}$, marked with $*$ in bottom spectrum $)$ via ${ }^{1} \mathrm{H}$ NMR spectroscopy upon adding 1 atm of $\mathrm{CO}_{2}$ to 3 in benzene- $d_{6}$. The ${ }^{1} \mathrm{H}$ NMR spectra of independently prepared dicarbonyl 1 (top spectrum) and bis(dinitrogen) compound $\mathbf{3}$ (middle spectrum) are provided for comparison. 
$30 \mathrm{~h}$

$18 \mathrm{~h}-\mathrm{l}, \mathrm{L}, \mathrm{L}$

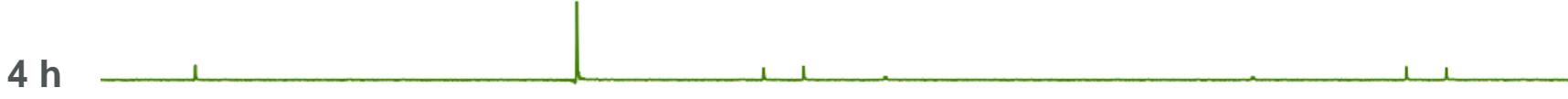

$0.25 \mathrm{~h}$

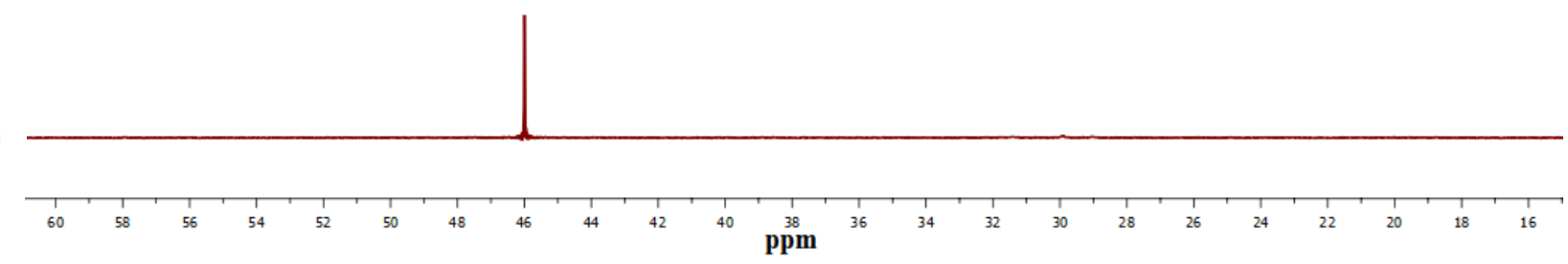

Figure S21. Observation of $\left({ }^{\mathrm{Ph} 2 \mathrm{PPr}} \mathrm{DI}\right) \mathrm{Mo}\left(\mathrm{CO}_{3}\right)(\mathrm{CO})(\mathbf{5})$ via time-dependent ${ }^{31} \mathrm{P}$ NMR spectroscopy upon adding $1 \mathrm{~atm}$ of $\mathrm{CO}_{2}$ to 3 in benzene- $d_{6}$. 


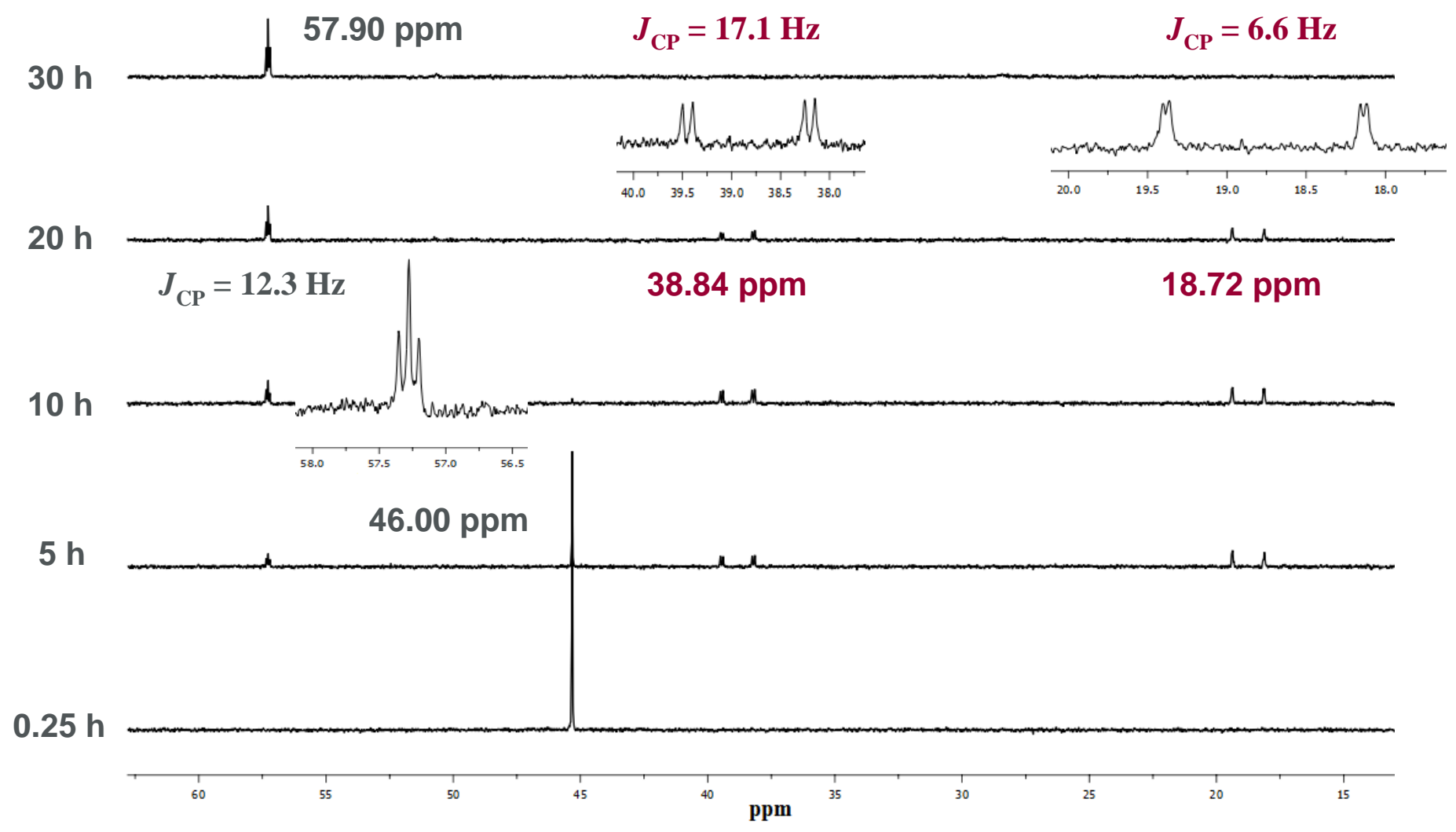

Figure S22. Time-dependent ${ }^{31} \mathrm{P}$ NMR spectra collected upon adding $0.5 \mathrm{~atm}$ of ${ }^{13} \mathrm{CO}_{2}$ to 3 in benzene- $d_{6}$. 


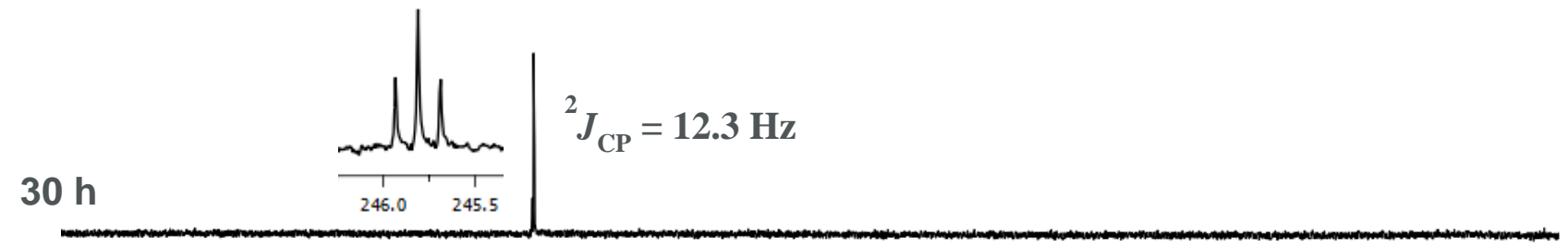

$25 \mathrm{~h}$
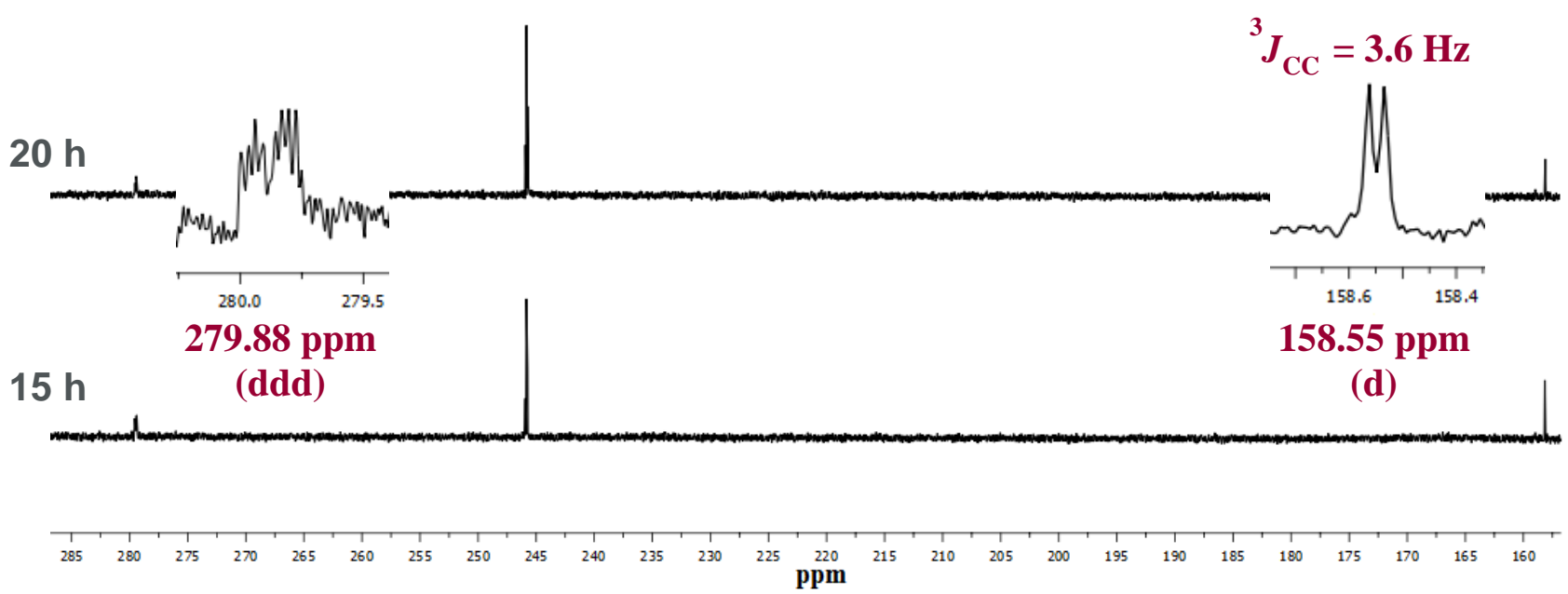

Figure S23. Time-dependent ${ }^{13} \mathrm{C}$ NMR spectra collected upon adding $0.5 \mathrm{~atm}$ of ${ }^{13} \mathrm{CO}_{2}$ to 3 in benzene- $d_{6}$. 


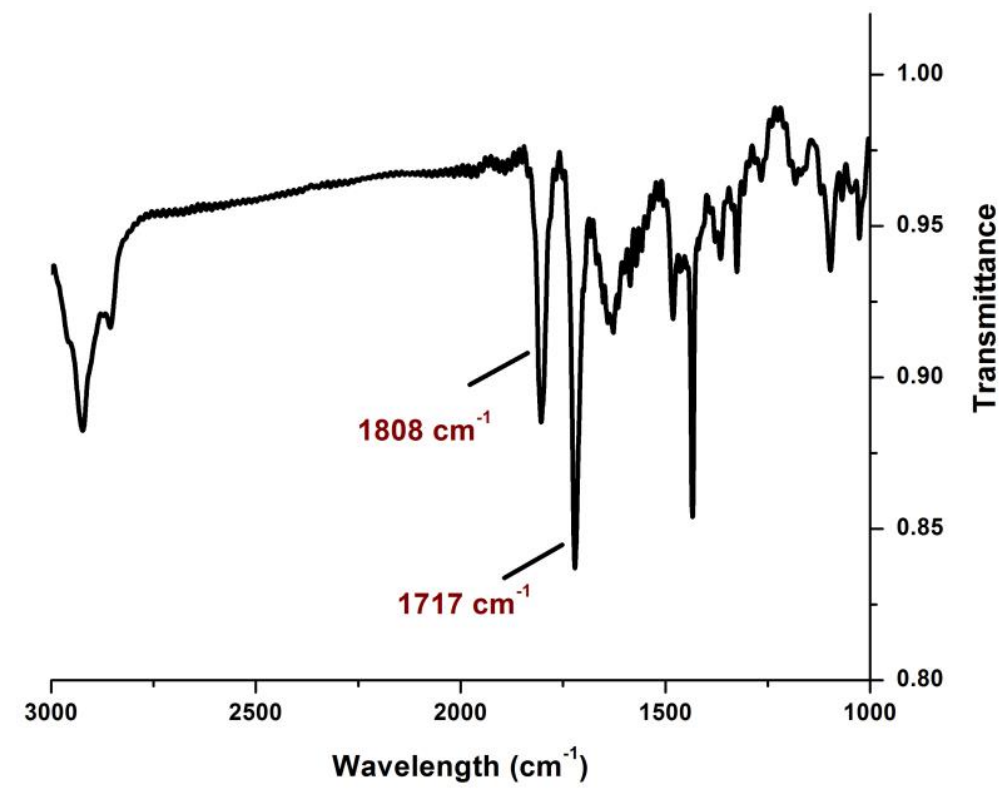

Figure S24. Infrared spectrum of $\left({ }^{\mathrm{Ph} 2 \mathrm{PPr}} \mathrm{DI}\right) \mathrm{Mo}\left({ }^{13} \mathrm{CO}\right)_{2}$ and 4 prepared following addition of ${ }^{13} \mathrm{CO}_{2}$ to $\left({ }^{\mathrm{Ph} 2 \mathrm{PPr}} \mathrm{DI}\right) \mathrm{Mo}\left(\mathrm{N}_{2}\right)_{2}$ in $\mathrm{KBr}$. 


\section{MOLECULAR ORBITAL CONTRIBUTIONS}

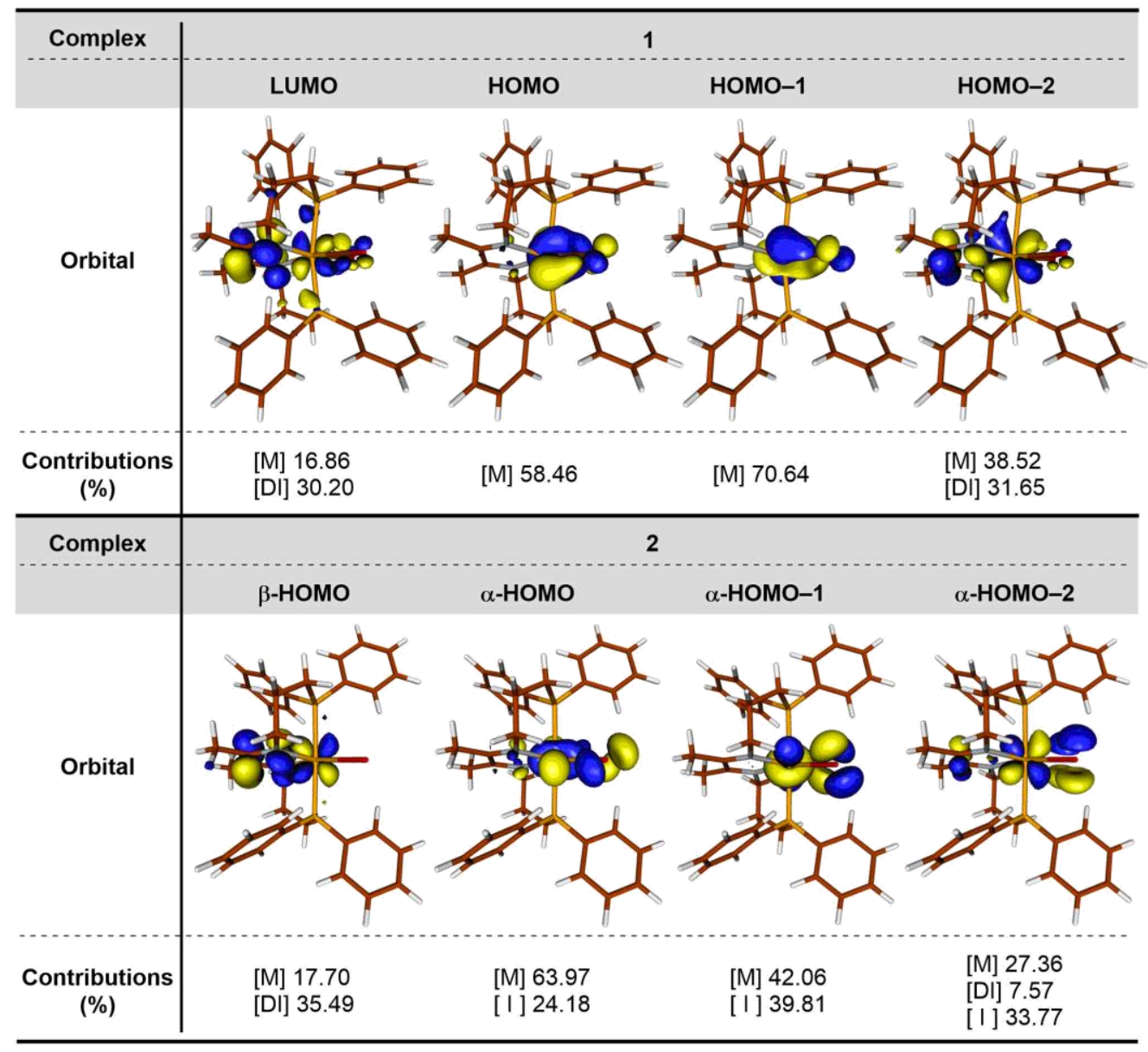

Figure S25. Percent contributions for the molecular orbitals of complex $\mathbf{1}$ and 2, where [M], $[\mathrm{DI}]$, and [I] indicate the Mo center, ${ }^{\mathrm{Ph} 2 \mathrm{PPr}} \mathrm{DI}$ chelate, and iodide ligands, respectively. 
EVALUATED DIMERIC SPECIES
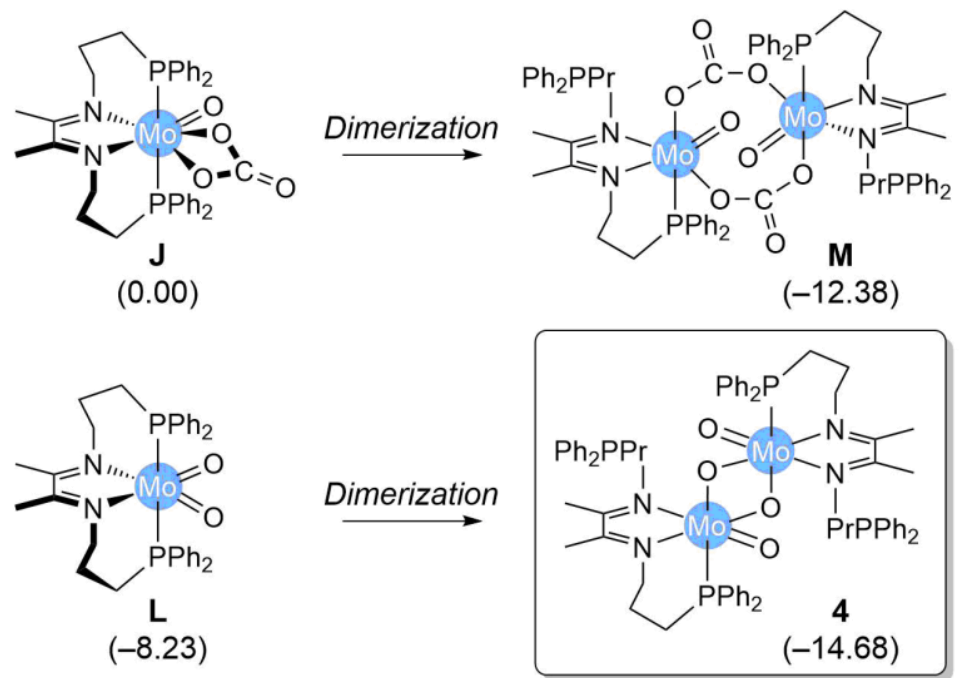

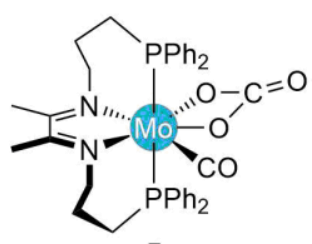

5

(24.14)

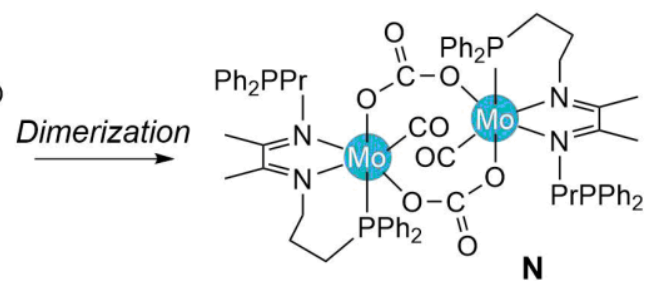

(40.07)

Figure S26. Examined dimeric structures and corresponding monomers. Relative free energies represented in $\mathrm{kcal} / \mathrm{mol}$.

The possibility that Mo(IV) oxo carbonate complex $\mathbf{J}$ dimerizes to form bridging carbonate complex $\mathbf{M}$ was evaluated. While dimerization of $\mathbf{J}$ shows an energy preference of 12.4 $\mathrm{kcal} / \mathrm{mol}$, the irreversible nature of $\mathrm{CO}_{2}$ extrusion would gradually increase the population of bis(oxo) intermediate $\mathbf{L}$ located at $8.2 \mathrm{kcal} / \mathrm{mol}$ lower than $\mathbf{J}$. Additionally, the dimerization of Mo(II) carbonyl carbonate complex 5 to generate bridging carbonate carbonyl dimer $\left[\left(\kappa^{3}-\right.\right.$ $\left.\left.{ }^{\mathrm{Ph} 2 \mathrm{PPr}} \mathrm{DI}\right) \mathrm{Mo}(\mathrm{CO})\left(\mu-\mathrm{CO}_{3}\right)\right]_{2}, \mathbf{N}$, was tested. The formation of this $\mathrm{Mo}(\mathrm{II})$-dimer is endergonic by $16.0 \mathrm{kcal} / \mathrm{mol}$, indicating that it loses $\mathrm{CO}_{2}$ to form $\mathrm{Mo}(\mathrm{IV})$ oxo compounds. Thus, we expect that the final product mixture would mainly consist of $\mathbf{4}$. During an intensive conformational search for $\mathbf{4}$, we found that there could be trans- and cis-isomers depending on the direction of the two oxo functionalities, and 4-cis was discarded because it is $3.80 \mathrm{kcal} / \mathrm{mol}$ higher in electronic energy. 
ENERGY COMPONENTS FOR THE DFT-OPTIMIZED STRUCTURES

Table S5. Computed Energy Components for Optimized Structures.

\begin{tabular}{|c|c|c|c|c|}
\hline & $\mathrm{E}(\mathrm{SCF}) /(\mathrm{eV})$ & ZPE/(kcal/mol) & $\mathrm{S}(\mathrm{gas}) /(\mathrm{cal} / \mathrm{mol} \cdot \mathbf{K})$ & $\mathrm{G}^{\text {solv }} /(\mathrm{kcal} / \mathrm{mol})$ \\
\hline & $\begin{array}{c}\text { B3LYP-D3 } \\
\text { /cc-pVTZ(-f)/LACV3P }\end{array}$ & $\begin{array}{c}\text { B3LYP-D3 } \\
/ 6-31 \mathrm{G}^{* *} / \mathrm{LACVP}\end{array}$ & $\begin{array}{c}\text { B3LYP-D3 } \\
\text { / 6-31G**/LACVP }\end{array}$ & $\begin{array}{c}\text { B3LYP-D3 } \\
/ 6-31 \mathrm{G}^{* * / L A C V P}\end{array}$ \\
\hline 1 & -65463.070 & 413.984 & 247.316 & -6.53 \\
\hline 2 & -59914.852 & 404.75 & 252.01 & -7.94 \\
\hline 3 & -65254.773 & 414.3 & 245.043 & -6.61 \\
\hline 4 & -126777.938 & 815.685 & 426.145 & -10.10 \\
\hline 4-cis & -126777.773 & 814.67 & 432.08 & -11.66 \\
\hline 5 & -69559.047 & 420.226 & 249.828 & -13.12 \\
\hline $\mathbf{F}$ & -69558.820 & 420.744 & 246.355 & -9.79 \\
\hline F-TS & -69558.016 & 418.876 & 255.682 & -8.24 \\
\hline $\mathbf{G}$ & -69558.172 & 418.60 & 247.04 & -10.27 \\
\hline G-TS & -69557.945 & 418.741 & 251.042 & -8.34 \\
\hline $\mathbf{H}$ & -73655.625 & 426.98 & 255.06 & -14.36 \\
\hline $\mathbf{I}$ & -73655.023 & 425.52 & 261.05 & -14.43 \\
\hline I-TS & -73654.648 & 424.95 & 259.38 & -13.32 \\
\hline $\mathbf{J}$ & -68522.031 & 417.00 & 249.15 & -12.87 \\
\hline $\mathbf{K}$ & -68521.820 & 416.01 & 254.70 & -12.92 \\
\hline K-TS & -68521.820 & 414.98 & 249.74 & -10.29 \\
\hline $\mathbf{L}$ & -63388.305 & 406.97 & 240.41 & -8.11 \\
\hline $\mathbf{M}$ & -137045.688 & 834.89 & 449.69 & -16.00 \\
\hline $\mathbf{N}$ & -139118.359 & 840.26 & 457.60 & -16.54 \\
\hline $\mathrm{CO}_{2}$ & -5133.566 & 7.287 & 51.16 & -1.28 \\
\hline $\mathbf{N}_{2}$ & -2981.407 & 3.518 & 45.784 & 0.00 \\
\hline $\mathbf{O}_{2}$ & -4091.962 & 2.377 & 49.008 & 0.00 \\
\hline $\mathrm{CO}$ & -3084.527 & 3.154 & 47.237 & 0.00 \\
\hline
\end{tabular}


Table S6. Cartesian coordinates for the optimized geometries.

\begin{tabular}{|c|c|c|c|}
\hline $\mathrm{CO}_{2}$ & & & \\
\hline $\mathrm{C}$ & 1.099317908 & 0.000000000 & -0.841882 \\
\hline $\mathrm{O}$ & 1.651157260 & -0.126832008 & 0.181027561 \\
\hline $\mathrm{O}$ & 0.547478557 & 0.126832008 & -1.86479 \\
\hline
\end{tabular}

$\mathrm{N}_{2}$

$\begin{array}{llll}\mathrm{N} & 0.436098039 & 0.000000000 & -3.174334288 \\ \mathrm{~N} & 0.436098039 & 0.000000000 & -4.279282093\end{array}$

$\mathbf{O}_{2}$

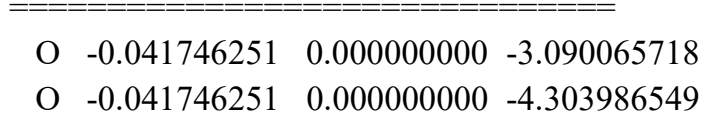

CO

$\begin{array}{lllll}\text { C } & -0.814051867 & 0.000000000 & -2.224912643\end{array}$

$\begin{array}{lllll}\text { O } & -0.814051867 & 0.000000000 & -3.363191366\end{array}$

1

\begin{tabular}{|c|c|c|c|}
\hline & -0.11231783 & 3.610190392 & 5.5030 \\
\hline$P$ & 0.346417159 & 3.326645613 & \\
\hline$P$ & -0.887148917 & 3.541485548 & 3.154784441 \\
\hline $\mathrm{O}$ & 0.300796688 & 0.485923171 & 5.386648178 \\
\hline $\mathrm{O}$ & -3.184185028 & 3.805869579 & 6.1879 \\
\hline $\mathrm{N}$ & 2.011855841 & 4.084692478 & 5.512878895 \\
\hline N & 0.0974 & 5.717124939 & 4.988 \\
\hline $\mathrm{C}$ & 3.816717625 & 5.813921452 & 5.422 \\
\hline $\mathrm{H}$ & 4.251029968 & 5.464718819 & 6.364179134 \\
\hline $\mathrm{H}$ & 3.890805960 & 6.901519775 & 5.428 \\
\hline $\mathrm{H}$ & 4.459410191 & 5.442863464 & 4.611 \\
\hline $\mathrm{C}$ & 2.385082960 & 5.348500252 & 5.272531986 \\
\hline $\mathrm{C}$ & 1.331990242 & 6.224905491 & 4.8741 \\
\hline $\mathrm{C}$ & 1.590044260 & 7.618942738 & 4.345306396 \\
\hline $\mathrm{H}$ & 1.027412534 & 7.804217339 & 3.425372601 \\
\hline $\mathrm{H}$ & 2.642009974 & 7.773454189 & 4.105193615 \\
\hline $\mathrm{H}$ & 1.297901750 & 8.396195412 & 5.065225124 \\
\hline $\mathrm{C}$ & 3.023548365 & 3.112004757 & 5.951475143 \\
\hline $\mathrm{H}$ & 4.009616852 & 3.354573250 & 5.538161278 \\
\hline
\end{tabular}

H $\quad 2.741039276 \quad 2.135986805 \quad 5.546635628$

C $\quad 3.1429660323 .0052511697 .486918926$

H $\quad 3.328373194 \quad 4.001242161 \quad 7.908913612$

$\begin{array}{llll}\mathrm{H} & 4.028075218 & 2.396353483 & 7.713938713\end{array}$

C $\quad 1.927087307 \quad 2.370376825 \quad 8.182765961$

$\begin{array}{llll}\mathrm{H} & 2.120635748 & 2.259883404 & 9.255258560\end{array}$

$\begin{array}{llll}\mathrm{H} & 1.745146751 & 1.368216872 & 7.779536247\end{array}$

C $\quad 0.660847306 \quad 4.846074581 \quad 8.924036026$

C $\quad 1.205423713 \quad 4.787122250 \quad 10.217864990$

H $\quad 1.404199600 \quad 3.825124025 \quad 10.682594299$

C $\quad 1.467581630 \quad 5.955578804 \quad 10.931543350$

H $\quad 1.884038329 \quad 5.895389080 \quad 11.933768272$

C $\quad 1.189650297 \quad 7.201434135 \quad 10.362211227$

H $\quad 1.394422412 \quad 8.111919403 \quad 10.919510841$

C $\quad 0.646243215 \quad 7.270528793 \quad 9.079574585$

$\begin{array}{llll}\mathrm{H} & 0.424905449 & 8.236126900 & 8.631678581\end{array}$

$\begin{array}{lllll}\text { C } & 0.385908365 & 6.099996090 & 8.363978386\end{array}$

$\begin{array}{llll}\mathrm{H} & -0.027618337 & 6.146181583 & 7.361598492\end{array}$

$\begin{array}{llll}\text { C } & -0.862860441 & 2.380534172 & 8.935203552\end{array}$

$\begin{array}{llll}\text { C } & -1.086817265 & 1.024373651 & 8.641544342\end{array}$

$\begin{array}{llll}\mathrm{H} & -0.537223816 & 0.537819445 & 7.841945171\end{array}$

$\begin{array}{llll}\text { C } & -2.037734747 & 0.294207484 & 9.352743149\end{array}$

H $\quad-2.195750475 \quad-0.754209101 \quad 9.113742828$

C $\quad-2.793444633 \quad 0.906536222 \quad 10.356182098$

$\begin{array}{lllll}\mathrm{H} & -3.539802551 & 0.337442487 & 10.903893471\end{array}$

$\begin{array}{lllll}\text { C } & -2.589394093 & 2.256536007 & 10.640831947\end{array}$

H $\quad-3.178964853 \quad 2.74773716911 .410742760$

C $\quad-1.630505443 \quad 2.989006042 \quad 9.937735558$

H $\quad-1.485694408 \quad 4.03883743310 .172403336$

C $-1.053398728 \quad 6.537672043 \quad 4.581936836$

$\begin{array}{llll}\mathrm{H} & -0.853097260 & 7.603872776 & 4.740205288\end{array}$

H $\quad-1.892611146 \quad 6.272578716 \quad 5.231433868$

C $-1.4708050496 .325816631 \quad 3.110944510$

H $\quad-0.608256042 \quad 6.502685070 \quad 2.455923080$

$\begin{array}{llll}\mathrm{H} & -2.218632460 & 7.090022087 & 2.861009598\end{array}$

C $-2.069846153 \quad 4.941936493 \quad 2.807986736$

$\begin{array}{llll}\mathrm{H} & -2.409517527 & 4.895598412 & 1.767699003\end{array}$

$\begin{array}{llll}\mathrm{H} & -2.943533421 & 4.763088226 & 3.443994284\end{array}$

C $\quad 0.367458671 \quad 3.775322676 \quad 1.818625450$

$\begin{array}{llll}\text { C } & 1.727417469 & 3.729019642 & 2.150088787\end{array}$

$\begin{array}{llll}\text { H } & 2.009260893 & 3.537434101 & 3.180686712\end{array}$

C $\quad 2.704158545 \quad 3.933153868 \quad 1.173182726$

H $\quad 3.755803585 \quad 3.890882254 \quad 1.445641398$

C $\quad 2.332319260 \quad 4.193219662-0.145673379$

$\begin{array}{lllll}\mathrm{H} & 3.091638327 & 4.354872227 & -0.906368494\end{array}$

C $\quad 0.977973640 \quad 4.241217613 \quad-0.487817317$

H $\quad 0.682017505 \quad 4.435642242-1.515529156$ 
$\begin{array}{llll}\text { C } & 0.003024133 & 4.033876419 & 0.486695319\end{array}$

$\begin{array}{llll}\mathrm{H} & -1.045206189 & 4.050785065 & 0.200703040\end{array}$

$\begin{array}{llll}\text { C } & -1.836148262 & 2.065979719 & 2.599274874\end{array}$

C $-1.332664609 \quad 1.171891570 \quad 1.644057035$

H $\quad-0.379276812 \quad 1.368321538 \quad 1.163886070$

$\begin{array}{llll}\text { C } & -2.045850754 & 0.019807661 & 1.306615710\end{array}$

H $\quad-1.638121367 \quad-0.6670763490 .569301486$

C $-3.271192312-0.250988841 \quad 1.914091229$

H $\quad-3.824889183-1.148732781 \quad 1.651798725$

$\begin{array}{llll}\text { C } & -3.778301001 & 0.632955194 & 2.869615316\end{array}$

H $\quad-4.728095055 \quad 0.425261557 \quad 3.355366230$

$\begin{array}{llll}\text { C } & -3.064864159 & 1.777938962 & 3.217257261\end{array}$

$\begin{array}{llll}\mathrm{H} & -3.457830906 & 2.431774855 & 3.989139318\end{array}$

C $\quad 0.086113036 \quad 1.639062047 \quad 5.449225903$

$\begin{array}{llll}\text { C } & -2.047484875 & 3.667422533 & 5.926639557\end{array}$

2

Mo $\quad 5.157725334 \quad 0.865199089 \quad 3.625309944$

$\begin{array}{llll}\mathrm{P} & 7.599326611 & 0.717282712 & 4.353625774\end{array}$

$\begin{array}{llll}\text { P } & 2.712020159 & 0.934187710 & 2.900225639\end{array}$

N $\quad 4.353714943 \quad-0.704001248 \quad 4.763290405$

$\begin{array}{llll}\mathrm{N} & 5.755575657 & -0.900939941 & 2.663373709\end{array}$

C $4.057250500-3.154314041 \quad 5.138703346$

H $\quad 2.971174717 \quad-3.123229980 \quad 5.269693375$

$\mathrm{H} \quad 4.509684563 \quad-3.178181887 \quad 6.138165951$

$\begin{array}{lllll}\mathrm{H} & 4.292495728 & -4.100416183 & 4.653826237\end{array}$

C $4.577913761 \quad-1.974026442 \quad 4.354678631$

$\begin{array}{llll}\text { C } & 5.374004364 & -2.084279299 & 3.197399378\end{array}$

C $\quad 5.732935905-3.395465851 \quad 2.540798664$

H $\quad 6.812895775 \quad-3.519301653 \quad 2.415161848$

H $\quad 5.275131226 \quad-3.461855650 \quad 1.545704007$

H $\quad 5.379560471 \quad-4.248872280 \quad 3.117348909$

C $\quad 6.734606743 \quad-0.915187359 \quad 1.565153241$

$\begin{array}{lllll}\mathrm{H} & 6.616051197 & 0.004058683 & 0.991481781\end{array}$

$\begin{array}{lllll}\mathrm{H} & 6.539376736 & -1.750593901 & 0.885102272\end{array}$

C $8.180534363-1.011679649 \quad 2.091765404$

H $\quad 8.284445763 \quad-1.927000046 \quad 2.683860302$

H $\quad 8.848892212-1.119352341 \quad 1.227625728$

$\begin{array}{llll}\text { C } & 8.658697128 & 0.200408667 & 2.912337065\end{array}$

$\begin{array}{llll}\mathrm{H} & 8.673479080 & 1.095274091 & 2.280162811\end{array}$

$\begin{array}{llll}\mathrm{H} & 9.681740761 & 0.042153392 & 3.272737265\end{array}$

C $3.389125586 \quad-0.484470636 \quad 5.851882935$

$\begin{array}{llll}\mathrm{H} & 3.621821880 & 0.468612999 & 6.327057362\end{array}$

$\begin{array}{llll}\mathrm{H} & 3.486721992 & -1.262005568 & 6.616216660\end{array}$

C $\quad 1.939405203 \quad-0.461710215 \quad 5.329135418$

H $\quad 1.268935561 \quad-0.395070285 \quad 6.195723057$

H $\quad 1.721002221 \quad-1.415269017 \quad 4.836331367$ $\begin{array}{llll}\text { C } & 1.607994080 & 0.705587626 & 4.381745815\end{array}$

$\mathrm{H} \quad 1.707203031 \quad 1.657517433 \quad 4.915257931$

$\begin{array}{llll}\mathrm{H} & 0.570557833 & 0.637214005 & 4.034334660\end{array}$

C $7.731231213-0.609126568 \quad 5.616934299$

$\begin{array}{llll}\text { C } & 6.880460739 & -0.509451091 & 6.731757641\end{array}$

$\begin{array}{llll}\mathrm{H} & 6.210485458 & 0.342192233 & 6.818881989\end{array}$

$\begin{array}{llll}\text { C } & 6.885179520 & -1.495163441 & 7.715302944\end{array}$

H $\quad 6.224680424-1.401589990 \quad 8.573257446$

$\begin{array}{llll}\text { C } & 7.732058048 & -2.601748466 & 7.596940517\end{array}$

H $\quad 7.729154110 \quad-3.375431538 \quad 8.359991074$

C $\quad 8.585323334-2.704916954 \quad 6.499098778$

H $\quad 9.252765656 \quad-3.557382584 \quad 6.405257225$

C $\quad 8.590306282-1.710439324 \quad 5.516714096$

H $\quad 9.273592949-1.8020474914 .678766727$

$\begin{array}{llll}\text { C } & 8.576722145 & 2.099028826 & 5.058590889\end{array}$

$\begin{array}{llll}\text { C } & 8.307769775 & 3.411049128 & 4.642550945\end{array}$

H $\quad 7.499374866 \quad 3.6033401493 .945705414$

C $\quad 9.069802284 \quad 4.469597816 \quad 5.138048649$

$\begin{array}{lllll}\mathrm{H} & 8.848784447 & 5.482511997 & 4.813933849\end{array}$

$\begin{array}{llll}\text { C } & 10.094064713 & 4.233324528 & 6.056787968\end{array}$

$\begin{array}{lllll}\mathrm{H} & 10.677597046 & 5.061853886 & 6.448783398\end{array}$

C $\quad 10.360577583 \quad 2.928537607 \quad 6.478385925$

H $\quad 11.151188850 \quad 2.737676382 \quad 7.199205399$

C $\quad 9.607413292 \quad 1.864935398 \quad 5.981808186$

$\begin{array}{llll}\mathrm{H} & 9.814040184 & 0.854848444 & 6.324256897\end{array}$

$\begin{array}{llll}\text { C } & 1.907228589 & 2.346104622 & 2.050841331\end{array}$

C $\quad 0.842410445 \quad 2.148085833 \quad 1.158387423$

$\begin{array}{llll}\mathrm{H} & 0.503821969 & 1.142099380 & 0.927466571\end{array}$

$\begin{array}{llll}\text { C } & 0.224213451 & 3.240188599 & 0.549780846\end{array}$

$\begin{array}{lllll}\text { H } & -0.594459474 & 3.077277899 & -0.146137267\end{array}$

$\begin{array}{llll}\text { C } & 0.661751211 & 4.536890984 & 0.828116417\end{array}$

H $\quad 0.183783233 \quad 5.386717796 \quad 0.348206013$

$\begin{array}{llll}\text { C } & 1.719516039 & 4.737898827 & 1.716137052\end{array}$

H $\quad 2.071861267 \quad 5.743218899 \quad 1.928182364$

$\begin{array}{llll}\text { C } & 2.346638918 & 3.649695635 & 2.323380470\end{array}$

H 3.1822271353 .8099288942 .995512962

$\begin{array}{llll}\text { C } & 2.401635408 & -0.487959623 & 1.780250669\end{array}$

$\begin{array}{llll}\text { C } & 3.237665892 & -0.604215622 & 0.655868590\end{array}$

$\begin{array}{llll}\mathrm{H} & 4.005958080 & 0.144662961 & 0.481084287\end{array}$

C $3.091535807-1.671729565-0.226272270$

H $\quad 3.741649866-1.745218158-1.094015598$

$\begin{array}{llll}\text { C } & 2.115273237 & -2.645881414 & 0.005257295\end{array}$

H $\quad 2.006460905-3.483088970-0.678928792$

C $\quad 1.276670933-2.534840822 \quad 1.113653779$

H $\quad 0.509594560 \quad-3.283132315 \quad 1.294331193$

C $\quad 1.414438486-1.457992196 \quad 1.994217038$

H $\quad 0.740916669-1.381396770 \quad 2.841644764$

I $\quad 5.895922184 \quad 2.679772854 \quad 1.483589053$

I $\quad 4.665810585 \quad 2.973939657 \quad 5.558565140$ 
3

\begin{tabular}{cccc}
\hline$========================$ \\
Mo & 5.187572002 & 0.881759048 & 3.656463861 \\
P & 7.555042267 & 0.807979107 & 4.413516998 \\
P & 2.826455355 & 1.006867409 & 2.891045809 \\
N & 4.313220501 & -0.762179852 & 4.759139061 \\
N & 5.848698616 & -0.956483006 & 2.727003336 \\
N & 5.853978157 & 2.090630293 & 2.170522213 \\
N & 6.237905025 & 2.676554680 & 1.273572803 \\
N & 4.673812389 & 2.300783634 & 5.009314060 \\
N & 4.361011982 & 3.013769150 & 5.839671612 \\
C & 3.907544613 & -3.215024471 & 5.013882160 \\
H & 2.822147846 & -3.101371288 & 5.094141483 \\
H & 4.295488358 & -3.363897800 & 6.030501842 \\
H & 4.085578918 & -4.135351181 & 4.458759785 \\
C & 4.550010204 & -2.019695997 & 4.349243164 \\
C & 5.460133553 & -2.129349947 & 3.254173994 \\
C & 5.941612244 & -3.453261614 & 2.707760572 \\
H & 7.031555653 & -3.483603001 & 2.615341425 \\
H & 5.524153233 & -3.648846626 & 1.710800529 \\
H & 5.658345699 & -4.286899567 & 3.349380970 \\
C & 6.865608692 & -0.959046423 & 1.664824367 \\
H & 6.704527378 & -0.076348506 & 1.039710879 \\
H & 6.760865688 & -1.834040284 & 1.012940288 \\
C & 8.302352905 & -0.921878397 & 2.228974342 \\
H & 8.449037552 & -1.784452558 & 2.889530659 \\
H & 8.998546600 & -1.044698954 & 1.388699889 \\
C & 8.669404030 & 0.372833312 & 2.977837801 \\
H & 8.581457138 & 1.232980728 & 2.303652287 \\
H & 9.713224411 & 0.342848927 & 3.311356544 \\
C & 3.308944702 & -0.537229657 & 5.809888363 \\
H & 3.579725266 & 0.375641972 & 6.347362041 \\
H & 3.309916019 & -1.350816250 & 6.544456959 \\
C & 1.883940935 & -0.379345179 & 5.236814022 \\
H & 1.184046626 & -0.327048302 & 6.081476212 \\
H & 1.624102473 & -1.278858304 & 4.666774273 \\
C & 1.677027225 & 0.867258906 & 4.357405186 \\
H & 1.878656030 & 1.773852348 & 4.940020084 \\
H & 0.634893894 & 0.935362995 & 4.024335384 \\
C & 7.915596008 & -0.483100653 & 5.684583664 \\
C & 6.971291065 & -0.660748899 & 6.708299160 \\
H & 6.077449799 & -0.043871239 & 6.715486526 \\
C & 7.160078049 & -1.627292037 & 7.694487572 \\
H & 6.418875694 & -1.745869279 & 8.480609894 \\
C & 8.291680336 & -2.447002649 & 7.668162346 \\
H & 8.434954643 & -3.207677841 & 8.430572510 \\
C & 9.235578537 & -2.283406258 & 6.653607368 \\
H & 10.119492531 & -2.915224075 & 6.624054432 \\
& & &
\end{tabular}

C $\quad 9.051181793-1.306382537 \quad 5.671738148$

H $\quad 9.802495956-1.194174528 \quad 4.895965576$

$\begin{array}{llll}\text { C } & 8.409974098 & 2.306597233 & 5.064074993\end{array}$

C $7.884163857 \quad 3.568624496 \quad 4.749858379$

H $\quad 6.967209816 \quad 3.637894869 \quad 4.173394680$

C $\quad 8.529353142 \quad 4.729646683 \quad 5.179947853$

H $\quad 8.109614372 \quad 5.700429440 \quad 4.930757523$

C $\quad 9.700870514 \quad 4.644575596 \quad 5.933916569$

$\begin{array}{llll}\mathrm{H} & 10.199225426 & 5.548513889 & 6.273326874\end{array}$

$\begin{array}{llll}\text { C } & 10.226292610 & 3.390841961 & 6.257292747\end{array}$

H $11.1340112693 .317108154 \quad 6.850670815$

$\begin{array}{llll}\text { C } & 9.585920334 & 2.229434967 & 5.825798988\end{array}$

H $\quad 9.996584892 \quad 1.259701490 \quad 6.093804836$

$\begin{array}{llll}\text { C } & 2.170277119 & 2.525809526 & 2.079680204\end{array}$

C $\quad 1.008701682 \quad 2.521354675 \quad 1.292404294$

H $\quad 0.481063306 \quad 1.590229869 \quad 1.106152177$

$\begin{array}{llll}\text { C } & 0.532219827 & 3.705669641 & 0.731426537\end{array}$

$\begin{array}{llll}\mathrm{H} & -0.365403920 & 3.689628124 & 0.118780829\end{array}$

$\begin{array}{llll}\text { C } & 1.209458232 & 4.907521248 & 0.949970007\end{array}$

$\begin{array}{llll}\mathrm{H} & 0.838983417 & 5.829077244 & 0.508827686\end{array}$

C $\quad 2.366793633 \quad 4.920751095 \quad 1.729241252$

H $\quad 2.9027509695 .851120949 \quad 1.895822287$

$\begin{array}{llll}\text { C } & 2.848273993 & 3.736279249 & 2.287867069\end{array}$

H $\quad 3.757173061 \quad 3.744712353 \quad 2.881323814$

$\begin{array}{llll}\text { C } & 2.296890259 & -0.352669477 & 1.758441329\end{array}$

C $3.192632437-0.7398260240 .749751806$

H $\quad 4.153170586 \quad-0.2386700810 .675954223$

C $2.869132042-1.764387250-0.138206318$

H $\quad 3.574849844-2.045252085-0.915651798$

C $\quad 1.647547245-2.433477402-0.024927316$

H $\quad 1.397707939-3.237962961-0.711070836$

C $\quad 0.750341713-2.0617730620 .977166951$

H $\quad-0.203125805-2.574664116 \quad 1.073558688$

C $\quad 1.071115255-1.027368188 \quad 1.860087872$

H $\quad 0.354047686 \quad-0.751435995 \quad 2.627298117$ 
H $\quad 5.035788059-0.309508622 \quad-3.628267527$

$\begin{array}{lllll}\mathrm{H} & 4.718727589 & -2.044336319 & -3.579406023\end{array}$

H $\quad 6.087837696-1.380819321-2.696491241$

$\begin{array}{llll}\text { O } & 1.239051223 & -1.110213757 & 1.670954227\end{array}$

$\begin{array}{llll}\text { O } & 1.758984089 & 1.330035448 & 0.116182581\end{array}$

Mo $\quad 0.461858243 \quad 1.780632377 \quad-1.301213503$

$\begin{array}{llll}\mathrm{N} & -1.379570961 & 2.164260626 & -2.262959003\end{array}$

$\begin{array}{llll}\mathrm{N} & -1.084531307 & 2.251948357 & 0.234163716\end{array}$

C $\quad-2.506934643 \quad 2.443557024-1.584693909$

$\begin{array}{llll}\text { C } & -2.315769434 & 2.551366329 & -0.181014657\end{array}$

C $-3.826952696 \quad 2.690400124-2.279124737$

$\begin{array}{lllll}\mathrm{H} & -4.654383183 & 2.731194735 & -1.570225358\end{array}$

$\mathrm{H} \quad-4.0723719601 .906376958-3.002381086$

$\begin{array}{lllll}\mathrm{H} & -3.820739746 & 3.643141031 & -2.827325344\end{array}$

C $\quad-3.399646521 \quad 2.984582663 \quad 0.780079901$

H $\quad-3.063796043 \quad 3.819678307 \quad 1.403121591$

$\begin{array}{llll}\mathrm{H} & -3.678128004 & 2.169123888 & 1.459986687\end{array}$

$\begin{array}{llll}\mathrm{H} & -4.303956985 & 3.308174849 & 0.265740544\end{array}$

$\begin{array}{lllll}\text { O } & 1.241170168 & 1.954569697 & -2.835337639\end{array}$

$\begin{array}{lllll}\text { O } & 0.192462221 & -0.187167674 & -1.131011486\end{array}$

H $\quad 2.865879536 \quad-0.725185871 \quad-4.000549793$

C $\quad 1.688815594-2.463657141-3.476300955$

C $2.190560341-1.034714341-3.196566105$

H $\quad 1.340231657 \quad-0.354406625 \quad-3.219786882$

H $\quad 2.516952276-3.181038141 \quad-3.398101091$

H $\quad 1.336959362-2.500732422-4.516504288$

$\begin{array}{lllll}\text { C } & 0.524912179 & -2.896111250 & -2.571725368\end{array}$

$\begin{array}{lllll}\text { P } & 0.983897984 & -3.013571024 & -0.785604775\end{array}$

H $\quad 0.092201889-3.840796947-2.909079790$

H $\quad-0.273190200 \quad-2.149211168-2.596093655$

$\begin{array}{llll}\text { C } & 3.221058130 & -4.365818977 & 0.198729694\end{array}$

$\begin{array}{llll}\text { C } & 2.145282745 & -4.430678844 & -0.699140429\end{array}$

C $\quad 2.015391350-5.566435814-1.515908837$

$\begin{array}{lllll}\mathrm{H} & 3.337642431 & -3.486106873 & 0.821354330\end{array}$

C $\quad 4.141189575-5.411746502 \quad 0.283698440$

H $\quad 4.968793869 \quad-5.343912125 \quad 0.985025644$

C $4.005393028-6.535484314-0.533580422$

$\begin{array}{lllll}\text { H } & 4.725393295 & -7.346950531 & -0.472893447\end{array}$

C $2.939219236-6.609333992-1.433534741$

H $\quad 2.826135874 \quad-7.480309010-2.073907852$

H $\quad 1.189930320-5.642529964-2.218314171$

C $\quad-1.541322351-2.590085983 \quad 0.264569998$

$\begin{array}{llll}\text { C } & -0.558706641 & -3.570355177 & 0.037967373\end{array}$

$\begin{array}{llll}\text { C } & -0.771261454 & -4.878174305 & 0.490998536\end{array}$

$\begin{array}{lllll}\text { H } & -1.371621728 & -1.571986079 & -0.079469018\end{array}$

$\begin{array}{llll}\text { C } & -2.720184803 & -2.926307917 & 0.927481174\end{array}$

H $\quad-3.474227428-2.162795544 \quad 1.101753235$

C $-2.929973602-4.233933926 \quad 1.375739336$

H $\quad-3.846626997 \quad-4.490714073 \quad 1.900492191$
C $\quad-1.954843044 \quad-5.207477093 \quad 1.155942202$

H $\quad-2.109821081 \quad-6.223909378 \quad 1.508771181$

$\begin{array}{llll}\mathrm{H} & -0.013397922 & -5.640052319 & 0.333585888\end{array}$

$\begin{array}{llll}\mathrm{H} & -1.585171580 & 2.126007318 & 2.290552139\end{array}$

$\begin{array}{llll}\text { C } & -0.199470118 & 3.763916016 & 2.025637865\end{array}$

$\begin{array}{llll}\text { C } & -0.730001807 & 2.369068623 & 1.650826216\end{array}$

H $\quad 0.046374071 \quad 1.630622745 \quad 1.859119654$

H $\quad-0.048205458 \quad 3.783579350 \quad 3.112456083$

$\begin{array}{llll}\mathrm{H} & -0.946812928 & 4.532100201 & 1.788443446\end{array}$

$\begin{array}{llll}\text { C } & 1.135391235 & 4.102375507 & 1.350238800\end{array}$

P $\quad 1.131123185 \quad 4.143709660 \quad-0.499712646$

$\mathrm{H} \quad 1.861805320 \quad 3.310652733 \quad 1.554981828$

$\begin{array}{llll}\mathrm{H} & 1.540269732 & 5.044378281 & 1.734745979\end{array}$

C $3.844774008 \quad 3.627934456 \quad-0.755453169$

$\begin{array}{llll}\text { C } & 2.869414806 & 4.638114452 & -0.839699984\end{array}$

$\begin{array}{llll}\text { C } & 3.260856390 & 5.955013275 & -1.115796924\end{array}$

H $\quad 3.539331675 \quad 2.606635809-0.538689315$

C $\quad 5.191251278 \quad 3.946091175 \quad-0.927768469$

H $\quad 5.939004898 \quad 3.161383629-0.849526584$

C $\quad 5.5811719895 .261952400-1.193149447$

H $\quad 6.631618023 \quad 5.505067348 \quad-1.329206467$

$\begin{array}{lllll}\text { C } & 4.612396717 & 6.262664318 & -1.292728662\end{array}$

H $\quad 4.905780792 \quad 7.286873817-1.508690715$

H $\quad 2.5154092316 .741485596-1.194078088$

C $\quad-0.654691875 \quad 5.478825569-2.177691221$

C $\quad 0.066899478 \quad 5.561866283 \quad-0.976625323$

$\begin{array}{llll}\text { C } & -0.088197611 & 6.701668262 & -0.171089038\end{array}$

H $\quad-0.558430731 \quad 4.590468884-2.794265509$

C $\quad-1.504820585 \quad 6.514369488-2.567032337$

$\begin{array}{lllll}\mathrm{H} & -2.057403803 & 6.434483051 & -3.499689341\end{array}$

C $-1.654608727 \quad 7.643355846-1.758884668$

H $\quad-2.323388815 \quad 8.445765495 \quad-2.058164120$

C $-0.943416774 \quad 7.734073639-0.559980392$

$\begin{array}{llll}\mathrm{H} & -1.055283427 & 8.608830452 & 0.075301893\end{array}$

$\begin{array}{lllll}\mathrm{H} & 0.457639486 & 6.789268970 & 0.764170587\end{array}$

$\begin{array}{lllll}\text { P } & -2.679293633 & -1.365611196 & -6.103331089\end{array}$

$\begin{array}{llll}\text { C } & -1.558432102 & -0.050481610 & -5.368831635\end{array}$

$\begin{array}{llll}\text { C } & -2.590923786 & -2.748321533 & -4.867498875\end{array}$

$\begin{array}{llll}\text { C } & -4.344050884 & -0.643368900 & -5.730443954\end{array}$

$\begin{array}{lllll}\text { C } & -1.803358316 & 0.394037485 & -3.917125463\end{array}$

$\mathrm{H} \quad-0.532770336-0.418227762-5.490071297$

$\begin{array}{lllll}\mathrm{H} & -1.640034437 & 0.800165594 & -6.058906078\end{array}$

$\begin{array}{llll}\text { C } & -1.978879213 & -3.939128637 & -5.289452076\end{array}$

$\begin{array}{llll}\text { C } & -3.119349957 & -2.696396828 & -3.564822197\end{array}$

$\begin{array}{lllll}\text { C } & -4.565417767 & 0.744444489 & -5.764717579\end{array}$

$\begin{array}{llll}\text { C } & -5.450330734 & -1.481083870 & -5.507533073\end{array}$

C $-1.469447136 \quad 1.883436918-3.701028824$

H $-0.499907255 \quad 2.114594698$-4.144610405

H $\quad-2.225969076 \quad 2.510952234-4.190780163$ 
H $\quad-1.196286917 \quad-0.197054729 \quad-3.226339579$

$\begin{array}{lllll}\mathrm{H} & -2.846150875 & 0.233197480 & -3.628908157\end{array}$

H $\quad-1.579943538 \quad-4.003977776 \quad-6.298989773$

$\begin{array}{llll}\text { C } & -1.893165350 & -5.045015335 & -4.438892365\end{array}$

$\begin{array}{llll}\text { C } & -3.031814337 & -3.796931982 & -2.713734627\end{array}$

H $\quad-3.618203163 \quad-1.797447920 \quad-3.218160152$

H $\quad-1.421979070 \quad-5.960236073-4.788764954$

$\begin{array}{lllll}\text { C } & -2.420114994 & -4.975232601 & -3.149190187\end{array}$

H $\quad-2.352029562 \quad-5.826971054 \quad-2.477808475$

H $\quad-3.424418688$-3.733900785 -1.704348922

H $\quad-3.739619017 \quad 1.427299261 \quad-5.940751553$

$\begin{array}{llll}\text { C } & -5.840124130 & 1.275660753 & -5.561771393\end{array}$

C $-6.723922253 \quad-0.950748622-5.304951668$

$\begin{array}{lllll}\mathrm{H} & -5.312089920 & -2.558233023 & -5.473177433\end{array}$

$\begin{array}{lllll}\mathrm{H} & -5.981733322 & 2.353334427 & -5.581827164\end{array}$

$\begin{array}{lllll}\text { C } & -6.925096035 & 0.430602729 & -5.327191830\end{array}$

H $\quad-7.917013645 \quad 0.843222260 \quad-5.163999081$

H $\quad-7.560966015 \quad-1.620243192 \quad-5.123636246$

$\begin{array}{lllll}\text { P } & 3.966137648 & 1.333652973 & 5.377785683\end{array}$

C $4.453261375 \quad-0.004280183 \quad 4.155868530$

$\begin{array}{llll}\text { C } & 4.586392403 & 2.805489779 & 4.440474510\end{array}$

$\begin{array}{llll}\text { C } & 2.132532358 & 1.433850408 & 5.145386696\end{array}$

$\begin{array}{llll}\text { C } & 4.179943085 & 0.239752442 & 2.659935951\end{array}$

H $\quad 5.521098614 \quad-0.186418772 \quad 4.326088905$

H $\quad 3.940744162 \quad-0.912181497 \quad 4.500757217$

C $\quad 5.936307430 \quad 3.152034760 \quad 4.612269402$

$\begin{array}{llll}\text { C } & 3.826646090 & 3.521016359 & 3.504099369\end{array}$

$\begin{array}{llll}\text { C } & 1.368426919 & 0.468742698 & 4.468694687\end{array}$

C $\quad 1.449189305 \quad 2.487133265 \quad 5.782257080$

C $\quad 4.105669022-1.101510644 \quad 1.915381432$

H $\quad 5.060040951-1.634310484 \quad 2.000357866$

H $\quad 3.342437983 \quad-1.714669704 \quad 2.401175976$

$\begin{array}{lllll}\mathrm{H} & 4.961423397 & 0.878827453 & 2.233751297\end{array}$

$\begin{array}{llll}\mathrm{H} & 3.236811638 & 0.768555641 & 2.501378298\end{array}$

$\begin{array}{llll}\mathrm{H} & 6.538045406 & 2.624059677 & 5.349451542\end{array}$

$\begin{array}{lllll}\text { C } & 6.519895077 & 4.157297611 & 3.839548826\end{array}$

$\begin{array}{llll}\text { C } & 4.409016609 & 4.524554729 & 2.729043722\end{array}$

$\begin{array}{llll}\mathrm{H} & 2.779556990 & 3.274768829 & 3.374362707\end{array}$

H $\quad 7.569081306 \quad 4.405773640 \quad 3.979293823$

$\begin{array}{llll}\text { C } & 5.759269238 & 4.839855671 & 2.888830423\end{array}$

$\begin{array}{llll}\mathrm{H} & 6.211936474 & 5.610932827 & 2.271426201\end{array}$

H $\quad 3.822084188 \quad 5.042967319 \quad 1.977488399$

H $\quad 1.830502868 \quad-0.350778878 \quad 3.933220863$

$\begin{array}{llll}\text { C } & -0.025724933 & 0.552731991 & 4.430340767\end{array}$

$\begin{array}{llll}\text { C } & 0.058141362 & 2.574972630 & 5.739195347\end{array}$

$\begin{array}{llll}\mathrm{H} & 2.013840437 & 3.247959852 & 6.317421436\end{array}$

H $\quad-0.576808512 \quad-0.197742224 \quad 3.870881796$

$\begin{array}{llll}\text { C } & -0.688776731 & 1.602677822 & 5.065382004\end{array}$

$\begin{array}{llll}\mathrm{H} & -1.773284197 & 1.668144226 & 5.035085678\end{array}$
H $\quad-0.443698585 \quad 3.399818897 \quad 6.239264488$ 
$\begin{array}{llll}\text { C } & 3.474123240 & -0.200726032 & 2.814884186\end{array}$

C $\quad 4.317276955-0.672105193 \quad 3.830581903$

H $\quad 4.798300266 \quad-1.641102552 \quad 3.736706734$

$\begin{array}{llll}\text { C } & 4.542342663 & 0.099874131 & 4.972917080\end{array}$

$\begin{array}{llll}\text { H } & 5.197431564 & -0.277034819 & 5.754383087\end{array}$

C $\quad 3.928175688 \quad 1.346411467 \quad 5.113955498$

H $\quad 4.106026649 \quad 1.945867181 \quad 6.002608776$

C $3.074280977 \quad 1.812576294 \quad 4.110356331$

H $\quad 2.593885183 \quad 2.781452179 \quad 4.206311703$

$\begin{array}{llll}\text { C } & 2.842668295 & 1.045702219 & 2.968548536\end{array}$

$\begin{array}{llll}\mathrm{H} & 2.177523136 & 1.411817908 & 2.190558672\end{array}$

$\begin{array}{llll}\text { C } & -6.317302704 & -3.858655214 & 0.956141055\end{array}$

$\begin{array}{llll}\text { C } & -7.443460941 & -4.028809547 & 0.134093434\end{array}$

H $\quad-7.589731693 \quad-3.382343054-0.727412105$

$\begin{array}{llll}\text { C } & -8.377058029 & -5.031626701 & 0.403433681\end{array}$

$\begin{array}{llll}\text { H } & -9.240769386 & -5.149121761 & -0.248425081\end{array}$

$\begin{array}{llll}\text { C } & -8.203808784 & -5.884005547 & 1.496032238\end{array}$

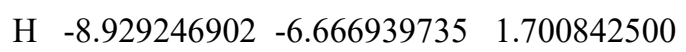

$\begin{array}{llll}\text { C } & -7.091121197 & -5.721067905 & 2.326460600\end{array}$

$\begin{array}{llll}\text { H } & -6.946251869 & -6.377172470 & 3.182739019\end{array}$

C $\quad-6.163786888$-4.713921547 2.064080238

$\begin{array}{llll}\mathrm{H} & -5.311434269 & -4.596217632 & 2.731403828\end{array}$

$\begin{array}{llll}\text { C } & -5.367953300 & -2.081720829 & -1.070893764\end{array}$

C $-6.012118816-0.865119696-1.346212745$

H $\quad-6.246302128 \quad-0.185914129-0.531810582$

$\begin{array}{lllll}\text { C } & -6.342726707 & -0.508775353 & -2.656172991\end{array}$

$\begin{array}{lllll}\text { H } & -6.843234539 & 0.437903374 & -2.841940880\end{array}$

$\begin{array}{llll}\text { C } & -6.005444050 & -1.349147439 & -3.718420267\end{array}$

H $\quad-6.253510475 \quad-1.069981933-4.739142418$

$\begin{array}{llll}\text { C } & -5.342807770 & -2.553855658 & -3.460646152\end{array}$

H $\quad-5.077048779$-3.215179443 -4.281740665

$\begin{array}{llll}\text { C } & -5.037209988 & -2.920276880 & -2.149244785\end{array}$

H $\quad-4.549060345 \quad-3.873355150 \quad-1.963800669$

O $\quad 0.156851545-1.261101723 \quad 1.972900152$

$\begin{array}{lllll}\text { O } & -0.857761204 & -0.010707157 & -0.464733630\end{array}$

P $\quad-2.455651045 \quad 1.854956269-2.137911797$

$\begin{array}{lllll}\text { Mo } & -0.126222461 & 1.700413823 & -1.032270670\end{array}$

$\begin{array}{llll}\mathrm{N} & 1.151867628 & 2.882758141 & -2.208183765\end{array}$

N $\quad 0.1887495520 .684618771 \quad-3.003610849$

$\begin{array}{llll}\text { C } & -2.948192835 & 0.321155816 & -3.038166761\end{array}$

$\begin{array}{lllll}\text { C } & -2.670794487 & 3.259856462 & -3.288919687\end{array}$

$\begin{array}{llll}\text { C } & -3.800041437 & 2.039652109 & -0.906850755\end{array}$

$\begin{array}{lllll}\text { P } & 4.595610619 & 4.352625370 & 1.568386436\end{array}$

$\begin{array}{llll}\text { C } & 3.663132668 & 4.785360813 & -0.007495528\end{array}$

C $\quad 5.712361813 \quad 5.833236694 \quad 1.658718824$

C $\quad 5.793134689 \quad 3.042470694 \quad 1.029575109$

C $\quad 1.606863976 \quad 2.471110582-3.407888651$

C $\quad 1.787524700 \quad 4.043071747 \quad-1.566945076$

$\begin{array}{lllll}\text { C } & 0.985994518 & 1.294127703 & -3.889417887\end{array}$ $\begin{array}{llll}\text { C } & -0.553343892 & -0.515285015 & -3.398963928\end{array}$

C $\quad 2.684780598 \quad 3.218205690-4.156076908$

H $\quad 3.5464119913 .420779228 \quad-3.510477304$

$\mathrm{H} \quad 2.336059570 \quad 4.185058594 \quad-4.542375565$

H $\quad 3.054164410 \quad 2.640077829 \quad-5.002725124$

C $\quad 1.2122722860 .741743088-5.277616501$

H $\quad 0.264407605 \quad 0.544168055 \quad-5.788064480$

H $\quad 1.761138797 \quad-0.210153833 \quad-5.245778084$

H $\quad 1.7782732251 .428503156-5.906851292$

H $\quad-0.716259420-1.104665875 \quad-2.496204615$

H $\quad 0.045042846-1.126775622-4.084314346$

$\begin{array}{llll}\text { C } & -1.918487430 & -0.211787090 & -4.042682648\end{array}$

$\begin{array}{lllll}\mathrm{H} & -1.794742703 & 0.493289351 & -4.874442577\end{array}$

H $-2.318422556-1.141405225-4.470101833$

H $-3.105284691 \quad-0.414552003 \quad-2.242276907$

H $\quad-3.923192978 \quad 0.478133649-3.506394625$

$\mathrm{H} \quad 1.018372178 \quad 4.562609673-0.989736676$

H $\quad 2.179370642 \quad 4.744261742 \quad-2.312777996$

C $\quad 2.913480043 \quad 3.593945980-0.613668144$

H $3.612495422 \quad 2.944990158-1.155175924$

H $2.4685313702 .967647791 \quad 0.164644867$

$\begin{array}{llll}\mathrm{H} & 2.939734697 & 5.551054478 & 0.300358683\end{array}$

H $\quad 4.324361324 \quad 5.259057522-0.743331432$

$\begin{array}{llll}\text { C } & -3.225310087 & 3.149711132 & -4.571402550\end{array}$

C $-2.203164101 \quad 4.512034416-2.852013350$

H $\quad-3.601033926 \quad 2.195132017 \quad-4.928104877$

C $\quad-3.312164545 \quad 4.270174980-5.402429581$

H $\quad-3.742714405 \quad 4.170167923-6.395532608$

C $\quad-2.849231720 \quad 5.510647297 \quad-4.961802959$

H $\quad-2.917668819 \quad 6.380443573 \quad-5.609299183$

$\begin{array}{llll}\text { C } & -2.303787947 & 5.628318310 & -3.679386377\end{array}$

$\begin{array}{lllll}\mathrm{H} & -1.943672061 & 6.591367245 & -3.327065945\end{array}$

H $\quad-1.753419995 \quad 4.596774578-1.865447521$

C $\quad-4.939143658 \quad 2.822321415-1.140164614$

$\begin{array}{llll}\text { C } & -3.667078257 & 1.354012370 & 0.311729997\end{array}$

H $\quad-5.045175076 \quad 3.363853693-2.076325893$

C $\quad-5.936196804 \quad 2.915223598 \quad-0.167273507$

H $\quad-6.815633297 \quad 3.526836872-0.352792233$

C $\quad-5.8026070592 .228351355 \quad 1.041604757$

H $\quad-6.578944206 \quad 2.303292990 \quad 1.798596382$

C $\quad-4.668394089 \quad 1.447902322 \quad 1.277324200$

$\begin{array}{llll}\mathrm{H} & -4.562102795 & 0.903688014 & 2.210727453\end{array}$

$\begin{array}{llll}\mathrm{H} & -2.780915260 & 0.752883673 & 0.496898234\end{array}$

$\begin{array}{llll}\text { C } & 7.108419895 & 5.742795467 & 1.767475605\end{array}$

C $\quad 5.124753475 \quad 7.110556602 \quad 1.700546265$

H $\quad 7.591257095 \quad 4.770560741 \quad 1.741476297$

$\begin{array}{llll}\text { C } & 7.890332699 & 6.892460823 & 1.900374889\end{array}$

$\begin{array}{llll}\mathrm{H} & 8.970512390 & 6.798348427 & 1.980688572\end{array}$

$\begin{array}{llll}\text { C } & 7.296246529 & 8.154845238 & 1.923334122\end{array}$ 


$\begin{array}{crrr}\mathrm{H} & 7.906740189 & 9.047977448 & 2.023837805 \\ \mathrm{C} & 5.906816483 & 8.258525848 & 1.822658539 \\ \mathrm{H} & 5.428843498 & 9.234689713 & 1.844505191 \\ \mathrm{H} & 4.043806553 & 7.213967323 & 1.638125420 \\ \mathrm{C} & 6.194780827 & 2.105845690 & 1.994921684 \\ \mathrm{C} & 6.355532169 & 2.958492041 & -0.255535483 \\ \mathrm{H} & 5.748090744 & 2.130004168 & 2.984425306 \\ \mathrm{C} & 7.151104927 & 1.131386757 & 1.695837140 \\ \mathrm{H} & 7.448744297 & 0.421243817 & 2.462973833 \\ \mathrm{C} & 7.698069572 & 1.055957556 & 0.413697004 \\ \mathrm{H} & 8.433986664 & 0.292429179 & 0.175201938 \\ \mathrm{C} & 7.290034294 & 1.968439698 & -0.565080583 \\ \mathrm{H} & 7.710829258 & 1.916423559 & -1.566097617 \\ \mathrm{H} & 6.070621967 & 3.676921844 & -1.019477010 \\ \mathrm{O} & -0.551682115 & 3.103486300 & -0.108570129 \\ \mathrm{O} & 1.373525620 & 0.892459750 & 0.032517660\end{array}$

5

\begin{tabular}{cccc}
\hline Mo & 4.885332584 & 1.117082715 & 4.086163044 \\
P & 7.360388756 & 0.840502441 & 4.320662975 \\
P & 2.666162252 & 1.001001716 & 2.835323334 \\
N & 3.930411100 & -0.505383432 & 5.125221729 \\
N & 5.388640881 & -0.700621903 & 3.034025192 \\
C & 3.540781498 & -2.953445911 & 5.398129940 \\
H & 2.449982882 & -2.959804773 & 5.299618244 \\
H & 3.769126892 & -2.953353882 & 6.470082760 \\
H & 3.903994799 & -3.894280434 & 4.988456249 \\
C & 4.168625355 & -1.756184578 & 4.722940922 \\
C & 5.018871307 & -1.862678885 & 3.575279474 \\
C & 5.351161480 & -3.187495708 & 2.930853128 \\
H & 6.355061054 & -3.191327810 & 2.503030777 \\
H & 4.641610622 & -3.406574488 & 2.122313738 \\
H & 5.308617592 & -4.008335590 & 3.645976067 \\
C & 6.119878292 & -0.693890095 & 1.758679509 \\
H & 5.849919319 & 0.230185315 & 1.243005753 \\
H & 5.803905964 & -1.523407459 & 1.118244767 \\
C & 7.645208359 & -0.736991704 & 1.953334570 \\
H & 7.914464951 & -1.660480857 & 2.476971149 \\
H & 8.116960526 & -0.785881281 & 0.963543713 \\
C & 8.206308365 & 0.475319177 & 2.712822199 \\
H & 8.063015938 & 1.388730168 & 2.124574423 \\
H & 9.284247398 & 0.371744335 & 2.878319979 \\
C & 2.806775808 & -0.251033723 & 6.030776978 \\
H & 2.959180355 & 0.709698319 & 6.518205643 \\
H & 2.743599653 & -1.015766501 & 6.813028812 \\
C & 1.469516873 & -0.234232217 & 5.249496460 \\
H & 0.670986533 & -0.036816761 & 5.975410461
\end{tabular}

H $\quad 1.277869225 \quad-1.238702774 \quad 4.857834816$

C $\quad 1.324211478 \quad 0.809489191 \quad 4.123735905$

$\begin{array}{llll}\mathrm{H} & 1.299132586 & 1.808771491 & 4.564089775\end{array}$

$\begin{array}{llll}\mathrm{H} & 0.384054989 & 0.646681309 & 3.583933115\end{array}$

C $7.768833160-0.5616483695 .440482140$

$\begin{array}{llll}\text { C } & 7.023902893 & -0.687533319 & 6.626053333\end{array}$

$\begin{array}{llll}\mathrm{H} & 6.265008926 & 0.055041000 & 6.855451107\end{array}$

C $7.265776634-1.749126554 \quad 7.496271133$

H $\quad 6.686323643 \quad-1.831522584 \quad 8.412141800$

$\begin{array}{llll}\text { C } \quad 8.245121002 & -2.699706078 & 7.198515415\end{array}$

H $\quad 8.427188873 \quad-3.528399229 \quad 7.877627850$

$\begin{array}{llll}\text { C } & 8.993548393 & -2.577489853 & 6.027963161\end{array}$

H $\quad 9.762755394 \quad-3.308274746 \quad 5.792290688$

C $8.758844376-1.5130844125 .154317856$

H $\quad 9.359404564 \quad-1.434100509 \quad 4.253479481$

$\begin{array}{llll}\text { C } & 8.350349426 & 2.242652655 & 4.967682838\end{array}$

$\begin{array}{llll}\text { C } & 7.798228264 & 3.527055979 & 5.049257278\end{array}$

$\begin{array}{llll}\mathrm{H} & 6.759703159 & 3.682503223 & 4.784189701\end{array}$

C $\quad 8.575089455 \quad 4.593633652 \quad 5.503269196$

H $\quad 8.134178162 \quad 5.584053516 \quad 5.570757389$

C $\quad 9.901524544 \quad 4.388313293 \quad 5.886215687$

$\begin{array}{llll}\mathrm{H} & 10.500370026 & 5.219936371 & 6.247441769\end{array}$

C $10.453188896 \quad 3.106550455 \quad 5.817999840$

H $\quad 11.4813098912 .936645269 \quad 6.126312733$

C $\quad 9.680992126 \quad 2.038200617 \quad 5.364023209$

H $\quad 10.110998154 \quad 1.040160298 \quad 5.337232113$

$\begin{array}{llll}\text { C } & 2.086558104 & 2.481758833 & 1.924408793\end{array}$

C $\quad 1.266842365 \quad 2.395546675 \quad 0.788592577$

$\begin{array}{llll}\mathrm{H} & 0.960926294 & 1.427143812 & 0.404832423\end{array}$

C $\quad 0.8492571123 .555166006 \quad 0.135657176$

H $\quad 0.220976785 \quad 3.478873968$-0.747762084

$\begin{array}{llll}\text { C } & 1.238948345 & 4.807473660 & 0.614176512\end{array}$

$\begin{array}{llll}\mathrm{H} & 0.917112589 & 5.709648132 & 0.100875460\end{array}$

$\begin{array}{llll}\text { C } & 2.040660381 & 4.897928238 & 1.753189802\end{array}$

H $\quad 2.342823744 \quad 5.870038033 \quad 2.132439137$

$\begin{array}{llll}\text { C } & 2.466999769 & 3.744121075 & 2.409870386\end{array}$

H 3.0757589343 .8103756903 .306441545

$\begin{array}{llll}\text { C } & 2.530345440 & -0.379102945 & 1.641173601\end{array}$

$\begin{array}{llll}\text { C } & 3.157395840 & -0.242998660 & 0.389477074\end{array}$

$\begin{array}{lllll}\mathrm{H} & 3.601099730 & 0.709254920 & 0.112335801\end{array}$

C $3.208423615-1.312555552-0.500954509$

H $\quad 3.693238258-1.186793923-1.465217233$

C $2.642134428-2.543784142-0.155220866$

H $\quad 2.686148405 \quad-3.379454851 \quad-0.847922862$

$\begin{array}{llll}\text { C } \quad 2.010604620 & -2.687319517 & 1.080513477\end{array}$

$\begin{array}{llll}\mathrm{H} & 1.555308104 & -3.635596514 & 1.353305817\end{array}$

C $\quad 1.954235673-1.613043666 \quad 1.972874045$

H $\quad 1.452260375-1.744258046 \quad 2.923755407$

$\begin{array}{llll}\text { C } & 5.517043591 & 2.438229799 & 2.764732599\end{array}$ 
$\begin{array}{llll}\text { O } & 3.513007641 & 2.491665602 & 5.049333572\end{array}$

C $\quad 4.284280777 \quad 2.648291826 \quad 6.127890587$

$\begin{array}{lllll}\text { O } & 4.005191326 & 3.319241047 & 7.104361057\end{array}$

$\begin{array}{lllll}\text { O } & 5.410200119 & 1.937774062 & 5.980846405\end{array}$

$\begin{array}{llll}\text { O } & 5.899040699 & 3.103803396 & 1.873325825\end{array}$

\begin{tabular}{|c|c|c|}
\hline & & \\
\hline Mo & $5.364005089 \quad 1.016823888$ & 3.44309163 \\
\hline $\mathrm{P}$ & $7.649027348 \quad 0.805663288$ & 6854 \\
\hline$P$ & $2.873613596 \quad 1.068373203$ & \\
\hline $\mathrm{N}$ & $4.496738434 \quad-0.656594992$ & 4.575540066 \\
\hline $\mathrm{N}$ & $5.984488010-0.775870621$ & 84350 \\
\hline $\mathrm{C}$ & $4.231255531-3.117013216$ & 0396 \\
\hline $\mathrm{H}$ & $3.152472019-3.057836771$ & 99102 \\
\hline $\mathrm{H}$ & $4.712812901-3.224953413$ & 19120 \\
\hline $\mathrm{H}$ & $4.418609142-4.031629086$ & 0862 \\
\hline $\mathrm{C}$ & $4.758801937-1.896115303$ & 9045 \\
\hline $\mathrm{C}$ & $54-1.977$ & 1525 \\
\hline $\mathrm{C}$ & $6.048578739-3.272293091$ & 4747 \\
\hline $\mathrm{H}$ & $46678-3.391285658$ & 7840 \\
\hline $\mathrm{H}$ & $21243-3.33148$ & 1894 \\
\hline $\mathrm{H}$ & $5-4.13$ & 4092 \\
\hline $\mathrm{C}$ & $99-0.76$ & 2024 \\
\hline $\mathrm{H}$ & $7.043480396 \quad 0.16$ & 5106 \\
\hline $\mathrm{H}$ & $6.965873718-1.590694308$ & 2114 \\
\hline $\mathrm{C}$ & $8.452854156-0.879448116$ & 15085 \\
\hline $\mathrm{H}$ & $8.482220650-1.808543086$ & 5007 \\
\hline $\mathrm{H}$ & $3658-0.97$ & 8079 \\
\hline $\mathrm{C}$ & $8.844343185 \quad 0.309614539$ & 7171 \\
\hline $\mathrm{H}$ & $8.924604416 \quad 1.217682242$ & 4345 \\
\hline $\mathrm{H}$ & $9.826472282 \quad 0.136597529$ & 9866 \\
\hline $\mathrm{C}$ & $3.602227211-0.504355848$ & 30097 \\
\hline $\mathrm{H}$ & $3.889789104 \quad 0.395$ & 8179 \\
\hline $\mathrm{H}$ & $7412-1.34$ & 7606 \\
\hline $\mathrm{C}$ & $2.129910231 \quad-0.380768239$ & 4510 \\
\hline $\mathrm{H}$ & $1.517453432-0.336311489$ & 58676 \\
\hline $\mathrm{H}$ & $1.822468519-1.280666351$ & 77346 \\
\hline $\mathrm{C}$ & $\begin{array}{ll}1.840780973 & 0.870330989\end{array}$ & 4.474286556 \\
\hline $\mathrm{H}$ & $2.059662819 \quad 1.769351721$ & 5.060784340 \\
\hline $\mathrm{H}$ & $\begin{array}{ll}0.782870531 & 0.922578037\end{array}$ & 86488 \\
\hline $\mathrm{C}$ & $7.687431335-0.470024973$ & 75528 \\
\hline $\mathrm{C}$ & $6.792363167-0.300929964$ & 6.872472286 \\
\hline $\mathrm{H}$ & $\begin{array}{ll}6.153206348 & 0.577425539\end{array}$ & 6.909925461 \\
\hline $\mathrm{C}$ & $6.709439278-1.262988925$ & 7.875631332 \\
\hline $\mathrm{H}$ & $6.014646053-1.120977521$ & 8.698886871 \\
\hline $\mathrm{C}$ & $7.507953644-2.409925699$ & 7.818414211 \\
\hline $\mathrm{H}$ & $7.434991837-3.164457083$ & 8.597061157 \\
\hline
\end{tabular}

$\begin{array}{llll}\text { C } & 8.401481628 & -2.580799580 & 6.761429787\end{array}$

H $\quad 9.029120445 \quad-3.466713905 \quad 6.715407848$

$\begin{array}{llll}\text { C } & 8.496133804 & -1.612034440 & 5.758545399\end{array}$

H $\quad 9.206001282-1.755387306 \quad 4.950288296$

$\begin{array}{llll}\text { C } & 8.458407402 & 2.275135040 & 5.220789909\end{array}$

C $\quad 8.282459259 \quad 3.504456043 \quad 4.564385891$

H $\quad 7.657892227 \quad 3.559070587 \quad 3.678460836$

$\begin{array}{llll}\text { C } & 8.886116028 & 4.654431820 & 5.071885109\end{array}$

$\begin{array}{llll}\mathrm{H} & 8.740518570 & 5.603419781 & 4.563569069\end{array}$

$\begin{array}{lllll}\text { C } & 9.654538155 & 4.593059063 & 6.236873627\end{array}$

$\begin{array}{llll}\mathrm{H} & 10.112780571 & 5.493959427 & 6.635456085\end{array}$

C $\quad 9.823745728 \quad 3.373089314 \quad 6.895024776$

H $\quad 10.415008545 \quad 3.320847273 \quad 7.805305481$

C $\quad 9.229649544 \quad 2.216195583 \quad 6.390204906$

H $\quad 9.358401299 \quad 1.271857023 \quad 6.911133766$

$\begin{array}{llll}\text { C } & 2.055100679 & 2.526778221 & 2.180995464\end{array}$

$\begin{array}{llll}\text { C } & 0.892805457 & 2.396810293 & 1.407674074\end{array}$

$\mathrm{H} \quad 0.492746443 \quad 1.412174940 \quad 1.183620930$

$\begin{array}{lllll}\text { C } & 0.252297342 & 3.529672861 & 0.905490279\end{array}$

$\begin{array}{llll}\mathrm{H} & -0.643259466 & 3.416971922 & 0.299967319\end{array}$

$\begin{array}{llll}\text { C } & 0.763779402 & 4.801097870 & 1.172819495\end{array}$

$\begin{array}{lllll}\mathrm{H} & 0.265500218 & 5.682625294 & 0.778712213\end{array}$

$\begin{array}{llll}\text { C } & 1.921834350 & 4.933585167 & 1.941377878\end{array}$

H $\quad 2.331009150 \quad 5.919176102 \quad 2.145567179$

$\begin{array}{llll}\text { C } & 2.571394205 & 3.804505348 & 2.441039085\end{array}$

H $3.4825143813 .912943602 \quad 3.020609140$

C $\quad 2.396457672-0.348682433 \quad 1.859176636$

$\begin{array}{llll}\text { C } & 3.212580919 & -0.669552982 & 0.762208104\end{array}$

$\begin{array}{llll}\mathrm{H} & 4.079257488 & -0.058021478 & 0.544348121\end{array}$

C $2.905599356-1.754281163-0.057130583$

H $\quad 3.547080278-1.983391643 \quad-0.904077590$

$\begin{array}{llll}\text { C } & 1.781388760 & -2.540805340 & 0.203610331\end{array}$

H $\quad 1.548268080-3.390957594-0.431954056$

$\begin{array}{llll}\text { C } & 0.954012275 & -2.222445965 & 1.279891372\end{array}$

H $\quad 0.069603667 \quad-2.819824696 \quad 1.485211611$

C $\quad 1.256886959-1.1321934462 .098619223$

H $\quad 0.589230061 \quad-0.897920072 \quad 2.920814991$

$\begin{array}{llll}\text { C } & 5.368348122 & 1.992988944 & 1.522220016\end{array}$

$\begin{array}{llll}C & 4.836151600 & 2.273386002 & 5.086946487\end{array}$

$\begin{array}{llll}\text { O } & 6.566248894 & 2.081109285 & 1.930324554\end{array}$

$\begin{array}{llll}\text { O } & 4.734886169 & 2.307131052 & 0.537753224\end{array}$

$\begin{array}{llll}\text { O } & 5.236851215 & 3.090082169 & 4.198153019\end{array}$

$\begin{array}{llll}\text { O } & 4.454911232 & 2.367876291 & 6.244759560\end{array}$

\section{F-TS}

$\begin{array}{llll}\text { Mo } & 5.115021706 & 0.675137520 & 3.547077417\end{array}$

P $7.499328136 \quad 0.720025182 \quad 4.178569794$ 
$\begin{array}{llll}\text { P } & 2.748356342 & 1.019149661 & 2.890367985\end{array}$

N $\quad 4.202286720 \quad-0.827555478 \quad 4.702132702$

$\begin{array}{llll}\mathrm{N} & 5.688623905 & -1.094677567 & 2.650722504\end{array}$

C $\quad 3.675149441 \quad-3.257037163 \quad 4.965557098$

H $\quad 2.596771955 \quad-3.080806017 \quad 5.037807465$

$\mathrm{H} \quad 4.044348240 \quad-3.427842617 \quad 5.986117840$

H $\quad 3.804899454 \quad-4.186935902 \quad 4.412001610$

$\begin{array}{llll}\text { C } & 4.381562710 & -2.104862928 & 4.292396069\end{array}$

C $\quad 5.264453888-2.257627726 \quad 3.196280479$

C $\quad 5.728953362-3.591158152 \quad 2.663825750$

$\begin{array}{llll}\mathrm{H} & 6.819423199 & -3.627514839 & 2.568786860\end{array}$

H $\quad 5.308214188 \quad-3.807671785 \quad 1.672331691$

H $\quad 5.443944931 \quad-4.410087109 \quad 3.324405193$

C $6.655019283-1.087535262 \quad 1.543270230$

H $\quad 6.423495770 \quad-0.219759688 \quad 0.918131828$

H $\quad 6.537707806 \quad-1.982127428 \quad 0.921814919$

C $\quad 8.122619629-0.985502601 \quad 2.013561249$

H $\quad 8.342049599 \quad-1.797708273 \quad 2.717889786$

H $\quad 8.763994217 \quad-1.144722819 \quad 1.137087703$

$\begin{array}{llll}\text { C } & 8.490948677 & 0.372247756 & 2.643429995\end{array}$

H $\quad 8.237205505 \quad 1.175999284 \quad 1.943871140$

H $\quad 9.565808296 \quad 0.422750473 \quad 2.849503756$

C $\quad 3.255547047 \quad-0.523650765 \quad 5.783527374$

$\begin{array}{llll}\mathrm{H} & 3.630200863 & 0.358573288 & 6.309874535\end{array}$

H $\quad 3.220247030 \quad-1.341892004 \quad 6.511515141$

C $\quad 1.826365709 \quad-0.237299204 \quad 5.277099609$

$\begin{array}{llll}\mathrm{H} & 1.172052622 & -0.144432738 & 6.153907299\end{array}$

H $\quad 1.461386085 \quad-1.095973372 \quad 4.700417519$

C $\quad 1.698302865 \quad 1.044541359 \quad 4.434430122$

$\mathrm{H} \quad 2.054342270 \quad 1.902243376 \quad 5.014332771$

$\begin{array}{llll}\mathrm{H} & 0.650536239 & 1.237333059 & 4.180376053\end{array}$

$\begin{array}{llll}\text { C } & 8.129368782 & -0.531358242 & 5.378237724\end{array}$

C $7.219724655-1.258189440 \quad 6.156530857$

H $\quad 6.158389091 \quad-1.079417706 \quad 6.028470039$

C $7.673350811-2.200811148 \quad 7.082029343$

$\begin{array}{llll}\mathrm{H} & 6.956173420 & -2.756458759 & 7.680771828\end{array}$

C $9.040100098-2.428475857 \quad 7.236867905$

H $\quad 9.393962860 \quad-3.162773848 \quad 7.955626488$

C $\quad 9.956150055-1.703884959 \quad 6.468657970$

$\begin{array}{llll}\text { H } & 11.022846222 & -1.870181799 & 6.593446732\end{array}$

C $\quad 9.504399300 \quad-0.760007799 \quad 5.548338890$

$\begin{array}{llll}\text { H } & 10.227032661 & -0.186913773 & 4.973831177\end{array}$

C $\quad 8.188174248 \quad 2.279081345 \quad 4.857017040$

C $\quad 8.817015648 \quad 3.242730618 \quad 4.057512760$

H $\quad 8.985585213 \quad 3.057602167 \quad 3.001496315$

$\begin{array}{llll}\text { C } & 9.216605186 & 4.462702751 & 4.606352329\end{array}$

H $\quad 9.699922562 \quad 5.201796055 \quad 3.973374367$

C $8.986241341 \quad 4.739371777 \quad 5.953980446$

H $\quad 9.287962914 \quad 5.693963528 \quad 6.375295639$ $\begin{array}{llll}\text { C } & 8.355154037 & 3.785710812 & 6.756458282\end{array}$

$\begin{array}{llll}\mathrm{H} & 8.163703918 & 3.994958162 & 7.805232525\end{array}$

$\begin{array}{llll}\text { C } \quad 7.960678577 & 2.565164566 & 6.213609695\end{array}$

H $\quad 7.468384743 \quad 1.829623222 \quad 6.844336987$

$\begin{array}{llll}\text { C } & 2.223417521 & 2.548463345 & 2.017592430\end{array}$

$\begin{array}{llll}\text { C } & 1.863501191 & 2.532899618 & 0.662174523\end{array}$

$\begin{array}{llll}\mathrm{H} & 1.820212245 & 1.591464281 & 0.123262271\end{array}$

C $\quad 1.557987928 \quad 3.720983028 \quad-0.001789466$

H $\quad 1.2813372613 .693005323-1.052433372$

C $\quad 1.608144522 \quad 4.938663960 \quad 0.677475810$

$\begin{array}{llll}\mathrm{H} & 1.373187184 & 5.863615990 & 0.157994464\end{array}$

$\begin{array}{llll}\text { C } & 1.970222354 & 4.964423656 & 2.025405407\end{array}$

H $\quad 2.024665594 \quad 5.908375740 \quad 2.560092926$

$\begin{array}{llll}\text { C } & 2.280858755 & 3.779911280 & 2.690030813\end{array}$

$\begin{array}{llll}\mathrm{H} & 2.580385208 & 3.828190327 & 3.731634617\end{array}$

C $\quad 1.978247643 \quad-0.324670702 \quad 1.884976268$

$\begin{array}{llll}\text { C } & 2.798157692 & -1.098843455 & 1.052628636\end{array}$

H $\quad 3.862616777 \quad-0.895608246 \quad 1.027701974$

$\begin{array}{llll}\text { C } \quad 2.257885456 & -2.121374846 & 0.271015257\end{array}$

H $\quad 2.908562660 \quad-2.710025787 \quad-0.370475054$

$\begin{array}{llll}\text { C } & 0.889846444 & -2.389358044 & 0.316879570\end{array}$

$\begin{array}{lllll}\mathrm{H} & 0.468569607 & -3.189292669 & -0.286036223\end{array}$

C $\quad 0.061541323 \quad-1.6215710641 .139027953$

H $\quad-1.006599665 \quad-1.818996429 \quad 1.172804713$

C $\quad 0.600436330-0.594418824 \quad 1.912872195$

$\begin{array}{llll}\mathrm{H} & -0.062356170 & 0.002405588 & 2.532132626\end{array}$

$\begin{array}{llll}\text { C } & 5.519612789 & 2.584406376 & 2.873703003\end{array}$

$\begin{array}{llll}\text { O } & 4.660043240 & 2.233133078 & 5.217322826\end{array}$

$\begin{array}{llll}\text { O } & 5.460508823 & 3.740515471 & 3.372474909\end{array}$

$\begin{array}{llll}\text { O } & 5.873083591 & 2.110234261 & 1.767980456\end{array}$

$\begin{array}{llll}\text { C } & 4.864700317 & 3.437822819 & 5.146544933\end{array}$

O $\quad 4.829875469 \quad 4.486217976 \quad 5.689513683$ 


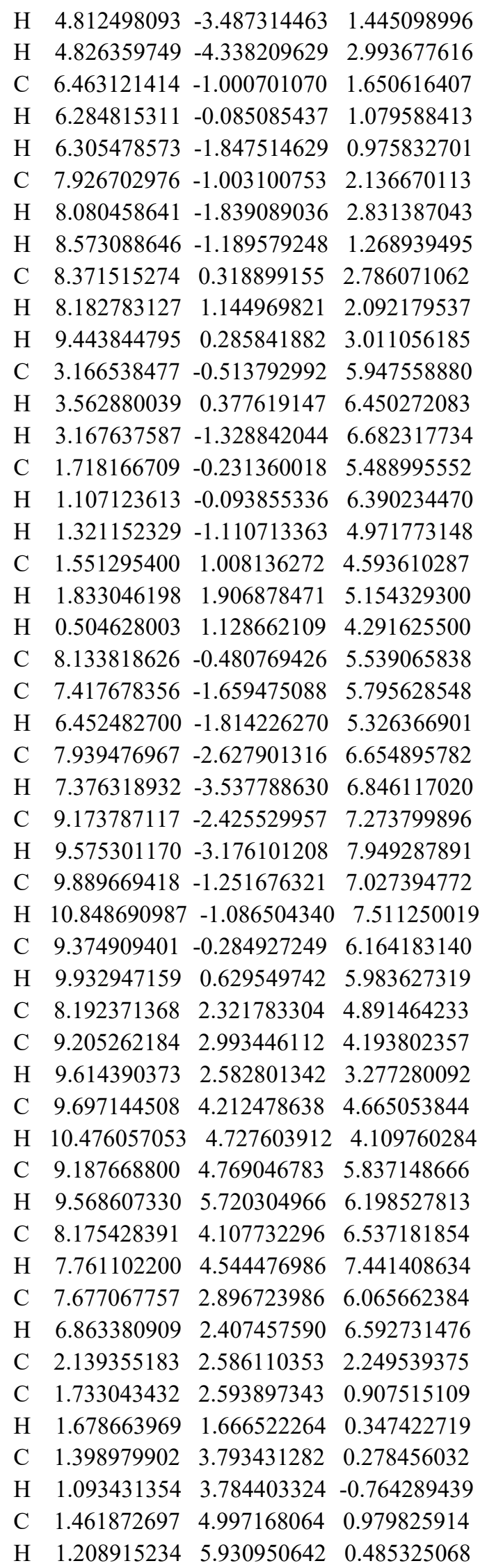

$\begin{array}{llll}\text { C } & 1.862534285 & 4.998163700 & 2.316730976\end{array}$

H $\quad 1.928120971 \quad 5.931922913 \quad 2.867754459$

$\begin{array}{llll}\text { C } & 2.203428745 & 3.804247618 & 2.948385715\end{array}$

H $\quad 2.543498755 \quad 3.832630396 \quad 3.978599072$

C $\quad 2.098153114-0.302287519 \quad 1.991033316$

C $\quad 2.950910807-0.652340233 \quad 0.930219650$

$\begin{array}{llll}\text { H } & 3.881000757 & -0.108397067 & 0.797009230\end{array}$

$\begin{array}{llll}\text { C } & 2.612197161 & -1.684002757 & 0.055845227\end{array}$

H $\quad 3.279011726-1.934260368-0.765166879$

$\begin{array}{llll}\text { C } & 1.423736215 & -2.396885395 & 0.238410190\end{array}$

H $\quad 1.164638042 \quad-3.209791183-0.434345394$

$\begin{array}{lllll}\text { C } & 0.567582488 & -2.053550005 & 1.286098838\end{array}$

H $\quad-0.363410473 \quad-2.595958710 \quad 1.428438067$

$\begin{array}{llll}\text { C } & 0.897648454 & -1.007107139 & 2.151325941\end{array}$

H $\quad 0.207596093 \quad-0.744109690 \quad 2.947248697$

$\begin{array}{llll}\text { O } & 6.073353291 & 3.878796101 & 1.294751525\end{array}$

C $\quad 5.715029716 \quad 3.111655951 \quad 2.153064251$

$\begin{array}{llll}\text { O } & 5.651922226 & 1.820948720 & 2.132196426\end{array}$

$\begin{array}{llll}\text { O } & 5.298116207 & 3.697969675 & 3.418217182\end{array}$

$\begin{array}{llll}\text { C } & 4.927777767 & 2.752828360 & 4.296176910\end{array}$

$\begin{array}{llll}\text { O } & 4.496805191 & 2.993244410 & 5.424023628\end{array}$ 
$\begin{array}{llll}\text { C } & 3.353465796 & -0.330492795 & 5.825720787\end{array}$

$\begin{array}{llll}\mathrm{H} & 3.582662582 & 0.624772668 & 6.294307232\end{array}$

H $\quad 3.407824993 \quad-1.087962866 \quad 6.613828659$

C $\quad 1.921453834-0.308208406 \quad 5.257677555$

H $\quad 1.223019481 \quad-0.224700600 \quad 6.100687504$

H $\quad 1.712693334-1.267745376 \quad 4.770694733$

$\begin{array}{lllll}\text { C } & 1.635412812 & 0.845461607 & 4.280754089\end{array}$

H $\quad 1.753998756 \quad 1.807156205 \quad 4.794024944$

$\begin{array}{llll}\mathrm{H} & 0.600135028 & 0.804485738 & 3.922308445\end{array}$

C $\quad 7.754088402 \quad-0.520499885 \quad 5.538681507$

C $\quad 6.926789284-0.422516763 \quad 6.671266556$

$\begin{array}{lllll}\mathrm{H} & 6.242719650 & 0.415648490 & 6.768249035\end{array}$

C $\quad 6.969006538-1.399952412 \quad 7.662344933$

H $\quad 6.326094151 \quad-1.307655454 \quad 8.533827782$

C $7.826503754-2.496959686 \quad 7.535243988$

$\begin{array}{llll}\text { H } & 7.849150658 & -3.264825821 & 8.303933144\end{array}$

C $8.656066895 \quad-2.598288536 \quad 6.419714928$

H $\quad 9.331323624 \quad-3.443653345 \quad 6.316312790$

C $\quad 8.624657631 \quad-1.611986518 \quad 5.429718018$

H $\quad 9.289579391 \quad-1.704312921 \quad 4.576886177$

C $\quad 8.587559700 \quad 2.162051201 \quad 4.986747265$

C $\quad 8.098048210 \quad 3.475463629 \quad 5.018158436$

H $\quad 7.109014988 \quad 3.705357075 \quad 4.642368793$

C $\quad 8.874079704 \quad 4.501750946 \quad 5.563385010$

H $\quad 8.478638649 \quad 5.513279438 \quad 5.583703518$

$\begin{array}{llll}\text { C } & 10.137614250 & 4.229976654 & 6.086244106\end{array}$

$\begin{array}{lllll}\text { H } & 10.735144615 & 5.029424667 & 6.515706539\end{array}$

$\begin{array}{llll}\text { C } & 10.628191948 & 2.920620441 & 6.067656517\end{array}$

$\begin{array}{lllll}\text { H } & 11.606571198 & 2.698205948 & 6.485354424\end{array}$

$\begin{array}{lllll}\text { C } & 9.858464241 & 1.894998074 & 5.523866653\end{array}$

$\begin{array}{lllll}\mathrm{H} & 10.237732887 & 0.876184523 & 5.538074493\end{array}$

C $\quad 2.073479176 \quad 2.399272680 \quad 1.917997003$

C $\quad 1.275570512 \quad 2.244882822 \quad 0.774328768$

$\begin{array}{lllll}\mathrm{H} & 1.058447957 & 1.252783537 & 0.390653461\end{array}$

C $\quad 0.759980321 \quad 3.363870144 \quad 0.119465686$

$\begin{array}{llll}\mathrm{H} & 0.146414682 & 3.231895447 & -0.767965257\end{array}$

C $\quad 1.032781482 \quad 4.646010876 \quad 0.598627806$

$\begin{array}{lllll}\mathrm{H} & 0.634523690 & 5.516088963 & 0.083599888\end{array}$

C $\quad 1.825541854 \quad 4.806685925 \quad 1.736341596$

$\begin{array}{llll}\mathrm{H} & 2.053048611 & 5.801646233 & 2.108510017\end{array}$

C $\quad 2.347207308 \quad 3.693161011 \quad 2.394232750$

H $\quad 2.991824150 \quad 3.827324152 \quad 3.257504225$

$\begin{array}{llll}\text { C } & 2.401146412 & -0.465374053 & 1.749272108\end{array}$

$\begin{array}{llll}\text { C } & 3.280275106 & -0.719486475 & 0.683437347\end{array}$

H $\quad 4.129659653 \quad-0.061562035 \quad 0.524300575$

C $3.076487780-1.801532149-0.169374049$

H $\quad 3.764425755-1.977935314-0.992105424$

C $\quad 1.992644310-2.659601688 \quad 0.037550662$

$\begin{array}{lllll}\text { H } & 1.836617708 & -3.510479927 & -0.619999170\end{array}$ $\begin{array}{llll}\text { C } & 1.109763265 & -2.415422201 & 1.088997483\end{array}$

H $\quad 0.260898471 \quad-3.074257135 \quad 1.251214147$

C $\quad 1.308325887-1.321447492 \quad 1.935619831$

H $\quad 0.600108266-1.1422699692 .738124132$

$\begin{array}{llll}\text { C } & 5.757285118 & 3.419393301 & 2.435896873\end{array}$

C $\quad 4.951443672 \quad 2.173683405 \quad 5.132467747$

O $\quad 5.896103382 \quad 2.305148125 \quad 1.921712041$

$\begin{array}{llll}\text { O } & 5.925165653 & 4.581429005 & 2.301578045\end{array}$

$\begin{array}{llll}\text { O } & 4.960232735 & 3.088690996 & 3.988872528\end{array}$

$\begin{array}{lllll}\text { O } & 4.850832939 & 2.535900593 & 6.275188923\end{array}$

$\begin{array}{llll}========================== \\ \mathbf{H} & & & \\ ====================== \\ \text { Mo } & 5.406681538 & 0.581965446 & 3.256298065 \\ \text { P } & 7.706948757 & 0.589705169 & 4.361519337 \\ \text { P } & 2.866985559 & 0.918046951 & 2.995900631 \\ \text { N } & 4.595307827 & -1.099778295 & 4.359971523 \\ \text { N } & 6.308433533 & -1.247723699 & 2.503711224 \\ \text { C } & 4.343025208 & -3.568772078 & 4.670736313 \\ \text { H } & 3.269772053 & -3.440693140 & 4.827126980 \\ \text { H } & 4.800461292 & -3.795970917 & 5.642313004 \\ \text { H } & 4.464785099 & -4.445257664 & 4.033991337 \\ \text { C } & 4.952333927 & -2.342589617 & 4.030907154 \\ \text { C } & 5.956290722 & -2.421768427 & 3.033493280 \\ \text { C } & 6.580096722 & -3.735039949 & 2.618688583 \\ \text { H } & 7.670969963 & -3.689955235 & 2.625252008 \\ \text { H } & 6.270880699 & -4.020013809 & 1.605111241 \\ \text { H } & 6.294815063 & -4.542372227 & 3.291284561 \\ \text { C } & 7.449039459 & -1.247983575 & 1.566600084 \\ \text { H } & 7.350997925 & -0.417321682 & 0.875893176 \\ \text { H } & 7.441987038 & -2.164843082 & 0.971417308 \\ \text { C } & 8.795021057 & -1.142191648 & 2.314085245 \\ \text { H } & 8.906069756 & -2.013600111 & 2.967936277 \\ \text { H } & 9.593684196 & -1.211623907 & 1.564955354 \\ \text { C } & 9.017683029 & 0.148147330 & 3.125243187 \\ \text { H } & 9.031426430 & 1.009939551 & 2.454078436 \\ \text { H } & 9.982312202 & 0.111655585 & 3.644180298 \\ \text { C } & 3.690717697 & -0.950492740 & 5.525434494 \\ \text { H } & 4.048579693 & -0.097687900 & 6.096869469 \\ \text { H } & 3.773430347 & -1.832013130 & 6.162170887 \\ \text { C } & 2.216686249 & -0.705830097 & 5.164669991 \\ \text { H } & 1.630117059 & -0.819952428 & 6.084785461 \\ \text { H } & 1.859260917 & -1.470505476 & 4.463610172 \\ \text { C } & 1.964726567 & 0.698200285 & 4.591972351 \\ \text { H } & 2.339385748 & 1.455556393 & 5.286940575 \\ \text { H } & 0.896388412 & 0.874641001 & 4.428426266 \\ \text { C } & 7.826785088 & -0.594326198 & 5.749176502 \\ \text { C } & 6.888987064 & -0.473152488 & 6.791170120\end{array}$


$\begin{array}{llll}\mathrm{H} & 6.174618244 & 0.342952818 & 6.766595364\end{array}$

C $\quad 6.876180172-1.393634796 \quad 7.836289406$

$\begin{array}{llll}\mathrm{H} & 6.147028446 & -1.287542820 & 8.635048866\end{array}$

C $7.794639587 \quad-2.447105885 \quad 7.859251499$

H $\quad 7.779367924 \quad-3.167231321 \quad 8.672828674$

C $\quad 8.739069939-2.563380003 \quad 6.839824677$

H $\quad 9.466284752 \quad-3.370510817 \quad 6.858970165$

C $8.759949684-1.639383435 \quad 5.792469501$

H $\quad 9.514122009 \quad-1.739313722 \quad 5.019704342$

C $\quad 8.234002113 \quad 2.206722975 \quad 5.014187813$

C $\quad 8.083492279 \quad 3.324409962 \quad 4.174768925$

$\begin{array}{llll}\text { H } & 7.643676281 & 3.204881668 & 3.190400839\end{array}$

C $\quad 8.449392319 \quad 4.587020874 \quad 4.636536121$

H $\quad 8.314433098 \quad 5.450096607 \quad 3.991483450$

C $\quad 8.958783150 \quad 4.746797085 \quad 5.926481724$

$\begin{array}{llll}\text { H } & 9.229802132 & 5.735873222 & 6.285608768\end{array}$

C $\quad 9.108834267 \quad 3.638026476 \quad 6.760358334$

H $\quad 9.499704361 \quad 3.759632826 \quad 7.766907215$

$\begin{array}{llll}\text { C } & 8.748184204 & 2.369237423 & 6.308714867\end{array}$

H $\quad 8.858543396 \quad 1.513412714 \quad 6.966924191$

$\begin{array}{llll}\text { C } & 2.373815060 & 2.546308041 & 2.354279995\end{array}$

C $\quad 2.329499960 \quad 2.749216318 \quad 0.965846181$

$\begin{array}{llll}\mathrm{H} & 2.565363884 & 1.933945656 & 0.291515738\end{array}$

C $\quad 2.008879423 \quad 4.004994392 \quad 0.455804765$

H $\quad 1.982650876 \quad 4.155745506 \quad-0.619641125$

C $\quad 1.738032103 \quad 5.066834927 \quad 1.321079373$

$\begin{array}{llll}\mathrm{H} & 1.492572069 & 6.046532631 & 0.919809043\end{array}$

C $\quad 1.800621033 \quad 4.872931480 \quad 2.701368570$

H $\quad 1.616742373 \quad 5.701156139 \quad 3.379653215$

C $\quad 2.123690367 \quad 3.620267630 \quad 3.220920086$

H $\quad 2.225643158 \quad 3.508482933 \quad 4.294768333$

C $\quad 2.047491789-0.302622974 \quad 1.904510975$

C $\quad 2.758834600-1.377483964 \quad 1.356827021$

H $\quad 3.824797153-1.456729293 \quad 1.529704094$

C $\quad 2.104620457-2.313996077 \quad 0.555832863$

$\begin{array}{lllll}\mathrm{H} & 2.666433811 & -3.139092207 & 0.126145750\end{array}$

C $\quad 0.740895391 \quad-2.183902502 \quad 0.290798336$

$\begin{array}{lllll}\text { H } & 0.236793429 & -2.909798622 & -0.341477841\end{array}$

C $\quad 0.027267640-1.110461831 \quad 0.828539073$

$\begin{array}{llll}\mathrm{H} & -1.032078028 & -0.996824443 & 0.615251422\end{array}$

$\begin{array}{llll}\text { C } & 0.676709056 & -0.173925802 & 1.631457448\end{array}$

$\begin{array}{llll}\mathrm{H} & 0.121628739 & 0.672818482 & 2.025974751\end{array}$

C $\quad 5.882955551 \quad 1.416831493 \quad 0.840779126$

$\begin{array}{lllll}\text { O } & 6.109454155 & 1.852167845 & -0.264699250\end{array}$

$\begin{array}{lllll}\text { O } & 4.758875370 & 0.819041550 & 1.239918828\end{array}$

$\begin{array}{lllll}\text { O } & 6.748516560 & 1.392970204 & 1.868055582\end{array}$

$\begin{array}{llll}\text { O } & 4.970449448 & 1.401329279 & 5.134139538\end{array}$

C $4.924158573 \quad 2.696753502 \quad 4.721861839$

$\begin{array}{lllll}\text { O } & 4.617424965 & 3.625474691 & 5.442755222\end{array}$ $\begin{array}{llll}\text { O } & 5.199651241 & 2.706350327 & 3.435723543\end{array}$

$\begin{array}{llll}========================== \\ \text { I } & & & \\ ======================= \\ \text { Mo } & 5.628448486 & 0.670509458 & 3.826976538 \\ \text { P } & 8.083942413 & 0.239867359 & 4.377773762 \\ \text { P } & 3.485553265 & 1.121117473 & 2.420998812 \\ \text { N } & 4.373291492 & -0.951594770 & 4.361337662 \\ \text { N } & 6.066970825 & -0.978101134 & 2.529245138 \\ \text { C } & 3.484401226 & -3.254277468 & 3.990952253 \\ \text { H } & 2.434658051 & -2.979151964 & 3.853318214 \\ \text { H } & 3.601688623 & -3.589844942 & 5.028372765 \\ \text { H } & 3.675496817 & -4.110481262 & 3.346637964 \\ \text { C } & 4.412646294 & -2.106214523 & 3.684976578 \\ \text { C } & 5.401349545 & -2.127607107 & 2.667285442 \\ \text { C } & 5.648794651 & -3.314367056 & 1.770462871 \\ \text { H } & 6.718038082 & -3.505057096 & 1.646786213 \\ \text { H } & 5.216714859 & -3.144228220 & 0.776601732 \\ \text { H } & 5.204887390 & -4.222994804 & 2.175083399 \\ \text { C } & 7.070910931 & -0.856686711 & 1.450442195 \\ \text { H } & 6.983971596 & 0.148156345 & 1.033746958 \\ \text { H } & 6.834197044 & -1.557313442 & 0.647619426 \\ \text { C } & 8.509504318 & -1.102921486 & 1.938233733 \\ \text { H } & 8.569594383 & -2.063505650 & 2.466682911 \\ \text { H } & 9.148490906 & -1.189137340 & 1.050805688 \\ \text { C } & 9.060422897 & 0.023028936 & 2.826626062 \\ \text { H } & 8.993266106 & 0.983733296 & 2.308151245 \\ \text { H } & 10.111986160 & -0.149822935 & 3.077041149 \\ \text { C } & 3.253567934 & -0.707914650 & 5.286010742 \\ \text { H } & 3.554172277 & 0.040686443 & 6.014906406 \\ \text { H } & 3.024828911 & -1.619208813 & 5.848618031 \\ \text { C } & 1.981962323 & -0.254330426 & 4.534974098 \\ \text { H } & 1.209559560 & -0.069213606 & 5.291756630 \\ \text { H } & 1.620094538 & -1.089301705 & 3.928419590 \\ \text { C } & 2.095446110 & 1.010311723 & 3.660818577 \\ \text { H } & 2.263454914 & 1.886119008 & 4.291397572 \\ \text { H } & 1.157101870 & 1.168696284 & 3.117498159 \\ \text { C } & 8.439093590 & -1.253428221 & 5.372897148 \\ \text { C } & 7.393793106 & -1.934661746 & 6.014900684 \\ \text { H } & 6.383957386 & -1.548855543 & 5.947779179 \\ \text { C } & 7.658264637 & -3.084842443 & 6.759012222 \\ \text { H } & 6.841804981 & -3.600670338 & 7.257637978 \\ \text { C } & 8.963066101 & -3.566501141 & 6.872622967 \\ \text { H } & 9.166017532 & -4.460240364 & 7.456423759 \\ \text { C } & 10.010344505 & -2.892035246 & 6.240750790 \\ \text { H } & 11.029661179 & -3.257256985 & 6.332760811 \\ \text { C } & 9.750571251 & -1.742914319 & 5.494984627 \\ \text { H } & 10.577005386 & -1.219015121 & 5.022971630\end{array}$




$\begin{array}{lccc}\text { C } & 8.833321571 & 1.663247228 & 5.229843616 \\ \text { C } & 8.828073502 & 2.916972399 & 4.589969158 \\ \text { H } & 8.396002769 & 3.040754557 & 3.601238728 \\ \text { C } & 9.338504791 & 4.030558586 & 5.255467415 \\ \text { H } & 9.326023102 & 4.996070385 & 4.757883072 \\ \text { C } & 9.844553947 & 3.912506342 & 6.551719189 \\ \text { H } & 10.234368324 & 4.786760807 & 7.066138268 \\ \text { C } & 9.836073875 & 2.672482967 & 7.191473961 \\ \text { H } & 10.214487076 & 2.577629089 & 8.205620766 \\ \text { C } & 9.328433990 & 1.550690532 & 6.537560940 \\ \text { H } & 9.309576035 & 0.594337702 & 7.049438953 \\ \text { C } & 3.235249996 & 2.762848616 & 1.674529314 \\ \text { C } & 2.628879786 & 2.921319008 & 0.420385689 \\ \text { H } & 2.302805424 & 2.053485632 & -0.144152731 \\ \text { C } & 2.465201378 & 4.196314812 & -0.116320685 \\ \text { H } & 2.004053116 & 4.313312531 & -1.093287230 \\ \text { C } & 2.906401873 & 5.316496372 & 0.591012895 \\ \text { H } & 2.790297270 & 6.308622837 & 0.163266122 \\ \text { C } & 3.509733200 & 5.160305977 & 1.839095116 \\ \text { H } & 3.875053167 & 6.025951862 & 2.383220673 \\ \text { C } & 3.677909851 & 3.889301300 & 2.385447502 \\ \text { H } & 4.170498371 & 3.769998074 & 3.344231129 \\ \text { C } & 3.138264656 & -0.100591987 & 1.105265260 \\ \text { C } & 4.064998150 & -0.203361854 & 0.052373365 \\ \text { H } & 4.903697491 & 0.487277120 & 0.018731343 \\ \text { C } & 3.889482260 & -1.165607095 & -0.940298855 \\ \text { H } & 4.608076572 & -1.230265617 & -1.753246069 \\ \text { C } & 2.797711611 & -2.038262129 & -0.896735072 \\ \text { H } & 2.669175625 & -2.792052984 & -1.669022560 \\ \text { C } & 1.865091443 & -1.927516222 & 0.134890810 \\ \text { H } & 1.003540635 & -2.588999748 & 0.166505411 \\ \text { C } & 2.029654980 & -0.958849609 & 1.127527833 \\ \text { H } & 1.279837728 & -0.875549436 & 1.905899644 \\ \text { C } & 6.481688976 & 2.684361458 & 1.482930183 \\ \text { O } & 6.846089363 & 3.811927080 & 1.781233907 \\ \text { O } & 6.646464825 & 1.767446876 & 2.673686981 \\ \text { O } & 6.069582462 & 2.097929001 & 0.478025615 \\ \text { O } & 4.538400650 & 2.052840471 & 4.895291805 \\ \text { C } & 5.105201244 & 1.789525628 & 6.095525742 \\ \text { O } & 5.953561306 & 0.772267401 & 5.906317711 \\ \text { O } & 4.854093552 & 2.338060617 & 7.139840126\end{array}$

\section{I-TS}

$\begin{array}{cccc}\text { Mo } & 5.451444149 & 0.662972927 & 3.333875418 \\ \text { P } & 7.733262062 & 0.631652355 & 4.467214108 \\ \text { P } & 2.900121450 & 0.960003674 & 2.911027670 \\ \text { N } & 4.550231457 & -1.115576625 & 4.372701168\end{array}$
$\begin{array}{llll}\mathrm{N} & 6.304672241 & -1.208888650 & 2.529715061\end{array}$

$\begin{array}{llll}\text { C } & 4.143634319 & -3.581964016 & 4.429298401\end{array}$

H $\quad 3.076618195 \quad-3.396827936 \quad 4.568264961$

H $\quad 4.548962116-3.947476149 \quad 5.381628990$

H $\quad 4.233310223 \quad-4.387232780 \quad 3.699087143$

C $\quad 4.851127625-2.332719564 \quad 3.953006029$

C $\quad 5.910575390-2.387495756 \quad 2.992056131$

C $\quad 6.528920174-3.699929476 \quad 2.564385653$

H $\quad 7.619990826-3.657899857 \quad 2.586483955$

H $\quad 6.233271599 \quad-3.965697050 \quad 1.541245699$

H $\quad 6.228938580-4.516757011 \quad 3.220063448$

C $7.479568005-1.181704164 \quad 1.638858438$

H $7.409589767 \quad-0.311077476 \quad 0.991818130$

H $7.489199638 \quad-2.0677993300 .998647332$

$\begin{array}{llll}\text { C } \quad 8.801512718 & -1.100458980 & 2.429112911\end{array}$

H $\quad 8.870645523 \quad-1.951200485 \quad 3.116071701$

H $\quad 9.621666908-1.210677147 \quad 1.708693624$

C $\quad 9.017783165 \quad 0.216918990 \quad 3.196252346$

$\begin{array}{llll}\mathrm{H} & 8.967649460 & 1.062202811 & 2.502474070\end{array}$

$\begin{array}{llll}\mathrm{H} & 10.006304741 & 0.232294887 & 3.668679476\end{array}$

C $3.600269556-0.992271185 \quad 5.501779556$

$\begin{array}{llll}\mathrm{H} & 3.992292881 & -0.212413192 & 6.150911331\end{array}$

H $\quad 3.583969116-1.924694896 \quad 6.070484638$

C $\quad 2.161123991 \quad-0.613193750 \quad 5.112259388$

$\begin{array}{llll}\mathrm{H} & 1.559368849 & -0.676545084 & 6.027451515\end{array}$

H $\quad 1.747089505-1.3487716914 .411835670$

$\begin{array}{lllll}\text { C } & 2.013460636 & 0.803789377 & 4.533026695\end{array}$

$\begin{array}{llll}\mathrm{H} & 2.434239149 & 1.542355776 & 5.221700191\end{array}$

$\begin{array}{llll}\mathrm{H} & 0.957047462 & 1.046284437 & 4.376020908\end{array}$

C $7.904015064-0.580232501 \quad 5.826852322$

$\begin{array}{llll}\text { C } & 6.925737381 & -0.577713549 & 6.836987972\end{array}$

$\begin{array}{llll}\mathrm{H} & 6.129723549 & 0.157659948 & 6.799159050\end{array}$

$\begin{array}{lllll}\text { C } & 6.983912468 & -1.506137609 & 7.874335289\end{array}$

H $\quad 6.222633839 \quad-1.490978122 \quad 8.649669647$

$\begin{array}{llll}\text { C } \quad 8.014524460 & -2.448172092 & 7.921228409\end{array}$

H $\quad 8.055668831 \quad-3.173494577 \quad 8.729315758$

C $\quad 8.998382568-2.446327209 \quad 6.932525635$

H $\quad 9.811652184 \quad-3.165994167 \quad 6.970483780$

C $\quad 8.947564125-1.514939904 \quad 5.893209457$

H $\quad 9.733624458-1.519834042 \quad 5.145515919$

$\begin{array}{llll}\text { C } & 8.282454491 & 2.231141806 & 5.143527985\end{array}$

C $\quad 8.085494995 \quad 3.372936964 \quad 4.348101616$

H $\quad 7.588570118 \quad 3.284001112 \quad 3.387209654$

C $\quad 8.485951424 \quad 4.620737553 \quad 4.822651386$

H $\quad 8.320640564 \quad 5.503050327 \quad 4.211439610$

C $\quad 9.070853233 \quad 4.740599155 \quad 6.084968090$

$\mathrm{H} \quad 9.369518280 \quad 5.717660904 \quad 6.455092430$

C $\quad 9.260519028 \quad 3.607984543 \quad 6.878348351$

H $\quad 9.7082929613 .699997902 \quad 7.864191532$ 
$\begin{array}{llll}\text { C } & 8.867609978 & 2.354182482 & 6.411877632\end{array}$

H $\quad 9.009114265 \quad 1.477603197 \quad 7.036565781$

$\begin{array}{llll}\text { C } & 2.388134003 & 2.573357105 & 2.231270790\end{array}$

$\begin{array}{llll}\text { C } & 2.182954073 & 2.718667030 & 0.850545168\end{array}$

$\begin{array}{llll}\mathrm{H} & 2.304229259 & 1.865076303 & 0.192590296\end{array}$

$\begin{array}{llll}\text { C } & 1.848160028 & 3.964303970 & 0.320712209\end{array}$

$\begin{array}{llll}\mathrm{H} & 1.694292188 & 4.067002773 & -0.749975860\end{array}$

C $\quad 1.723194838 \quad 5.074837685 \quad 1.157971740$

H $\quad 1.466985106 \quad 6.045578003 \quad 0.742226243$

C $\quad \begin{array}{llll}1.946388125 & 4.936172485 & 2.529107571\end{array}$

$\mathrm{H} \quad 1.874481559 \quad 5.799016953 \quad 3.185230255$

C $\quad 2.285604715 \quad 3.695934534 \quad 3.068501711$

H $\quad 2.506222486 \quad 3.628937244 \quad 4.128722191$

C $\quad 2.026426554-0.301876664 \quad 1.903797388$

C $2.703020334-1.420895696 \quad 1.402373910$

H $\quad 3.775050640-1.496742606 \quad 1.529069543$

$\begin{array}{llll}\text { C } & 2.007696629 & -2.409148932 & 0.704435647\end{array}$

$\begin{array}{lllll}\mathrm{H} & 2.545494318 & -3.268969297 & 0.313650489\end{array}$

$\begin{array}{llll}\text { C } & 0.633613110 & -2.287674427 & 0.491269112\end{array}$

$\begin{array}{lllll}\mathrm{H} & 0.095869593 & -3.054033041 & -0.059966616\end{array}$

C $-0.045348294-1.167647123 \quad 0.977332354$

$\mathrm{H} \quad-1.112128496 \quad-1.057351232 \quad 0.803024650$

C $\quad 0.645713925-0.182101339 \quad 1.681724906$

$\begin{array}{llll}\mathrm{H} & 0.113060646 & 0.694151223 & 2.041415453\end{array}$

$\begin{array}{llll}\text { C } & 5.675560951 & 1.211900711 & 0.458917797\end{array}$

$\begin{array}{lllll}\text { O } & 6.393799305 & 1.640364766 & -0.393447310\end{array}$

$\begin{array}{llll}\text { O } & 4.586691856 & 0.687700927 & 0.660571337\end{array}$

$\begin{array}{llll}\text { O } & 6.471505642 & 1.358660817 & 1.973915696\end{array}$

$\begin{array}{lllll}\text { O } & 5.017559052 & 1.297672033 & 5.256706238\end{array}$

C $\quad 4.848176479 \quad 2.625251770 \quad 4.970068932$

$\begin{array}{llll}\text { O } & 4.396605015 & 3.419503927 & 5.777325630\end{array}$

$\begin{array}{llll}\text { O } & 5.150044441 & 2.795709133 & 3.711907625\end{array}$

J

$\begin{array}{llll}\text { Mo } & 5.544197559 & 0.589840651 & 3.528381586\end{array}$

$\begin{array}{lllll}\mathrm{P} & 7.999908447 & 0.285768062 & 4.260267258\end{array}$

$\begin{array}{llll}\text { P } & 3.333098888 & 1.105217338 & 2.397319317\end{array}$

N $\quad 4.356437683 \quad-1.173135757 \quad 4.123860359$

$\begin{array}{llll}\mathrm{N} & 6.179894924 & -1.285858512 & 2.349394321\end{array}$

C $3.585398197 \quad-3.538058281 \quad 3.777829170$

H $\quad 2.551710129 \quad-3.234004736 \quad 3.946914673$

H $\quad 3.904172659 \quad-4.117833138 \quad 4.654914379$

H $\quad 3.581547499 \quad-4.213233948 \quad 2.920138597$

$\begin{array}{llll}\text { C } \quad 4.492619514 & -2.352176428 & 3.535198212\end{array}$

$\begin{array}{llll}\text { C } & 5.605231285 & -2.437148094 & 2.635486603\end{array}$

$\begin{array}{llll}\text { C } & 6.061615467 & -3.767970562 & 2.078804731\end{array}$

H $\quad 7.151889801 \quad-3.836148500 \quad 2.072145939$
H $\quad 5.712696075 \quad-3.914412260 \quad 1.048346162$

H $\quad 5.690001965-4.600221157 \quad 2.678108692$

C $\quad 7.275546551 \quad-1.272140265 \quad 1.363538742$

H $7.153505802-0.359341502 \quad 0.780343175$

H $7.187244415 \quad-2.1278231140 .687990546$

C $8.683265686-1.264812350 \quad 1.983483315$

H $\quad 8.816047668$-2.132512093 2.642216921

H $\quad 9.399551392-1.382017493 \quad 1.160494566$

$\begin{array}{llll}\text { C } & 9.035017967 & 0.026921386 & 2.739825249\end{array}$

$\begin{array}{llll}\mathrm{H} & 8.872519493 & 0.899481833 & 2.098921299\end{array}$

$\begin{array}{lllll}\mathrm{H} & 10.088623047 & 0.022526672 & 3.038687944\end{array}$

$\begin{array}{llll}\text { C } & 3.235076189 & -0.970270157 & 5.052430630\end{array}$

H $3.547251225 \quad-0.201188117 \quad 5.761369705$

H $\quad 3.032742262-1.888562083 \quad 5.613633633$

C $\quad 1.944539428 \quad-0.5224432354 .335370064$

H $\quad 1.141710281 \quad-0.505208910 \quad 5.083487511$

H $\quad 1.663731694-1.276527524 \quad 3.591875315$

$\begin{array}{llll}\text { C } & 1.998455048 & 0.864784479 & 3.667647600\end{array}$

H $\quad 2.215101719 \quad 1.634636283 \quad 4.416694164$

H $\quad 1.028168321 \quad 1.1104980713 .221080780$

C $\quad 8.349466324-1.205594659 \quad 5.271480083$

C $7.284439087-1.941369057 \quad 5.807196140$

H $\quad 6.267316818$-1.606103659 5.633442879

$\begin{array}{llll}\text { C } & 7.528918266 & -3.096068621 & 6.553151608\end{array}$

H $\quad 6.694541931 \quad-3.6572446826 .965701103$

$\begin{array}{llll}\text { C } & 8.837474823 & -3.527203798 & 6.768689156\end{array}$

H $\quad 9.027448654-4.427221775 \quad 7.347266674$

$\begin{array}{llll}\text { C } & 9.906476021 & -2.795602798 & 6.243782043\end{array}$

H $\quad 10.927991867 \quad-3.123146057 \quad 6.416551590$

C $9.664958954-1.640300512 \quad 5.502878666$

H $\quad 10.503136635 \quad-1.068273544 \quad 5.114673615$

$\begin{array}{llll}\text { C } & 8.837985039 & 1.662930489 & 5.125344276\end{array}$

C $\quad 9.109631538 \quad 2.854634762 \quad 4.430388927$

$\begin{array}{llll}\mathrm{H} & 8.819603920 & 2.981819630 & 3.392414808\end{array}$

C $\quad 9.733275414 \quad 3.913254976 \quad 5.089605331$

H $\quad 9.939215660 \quad 4.828875065 \quad 4.542448044$

C $10.075904846 \quad 3.809458733 \quad 6.439346790$

H $10.556901932 \quad 4.641353607 \quad 6.946643353$

C $\quad 9.788853645 \quad 2.634973764 \quad 7.135946274$

$\begin{array}{llll}\text { H } & 10.043807983 & 2.546360493 & 8.188590050\end{array}$

$\begin{array}{llll}\text { C } & 9.172426224 & 1.566231966 & 6.484210968\end{array}$

$\begin{array}{llll}\mathrm{H} & 8.957444191 & 0.656738400 & 7.036684990\end{array}$

$\begin{array}{llll}\text { C } & 3.004791260 & 2.774329185 & 1.731607080\end{array}$

$\begin{array}{llll}\text { C } & 2.112245798 & 2.977921009 & 0.667298496\end{array}$

H $1.622196198 \quad 2.131090641 \quad 0.195321366$

$\begin{array}{lllll}\text { C } & 1.865643859 & 4.267183304 & 0.199797362\end{array}$

H $\quad 1.179350972 \quad 4.418472290 \quad-0.629199684$

C $\quad 2.508852482 \quad 5.359219074 \quad 0.787440896$

H $2.323975325 \quad 6.362775326 \quad 0.413506716$ 
$\begin{array}{llll}\text { C } & 3.399029255 & 5.159375668 & 1.842741966\end{array}$

$\begin{array}{llll}\text { H } & 3.917402506 & 6.003586769 & 2.288024426\end{array}$

$\begin{array}{llll}\text { C } & 3.652725458 & 3.872318983 & 2.319101572\end{array}$

H $\quad 4.386381149 \quad 3.714358807 \quad 3.104019642$

C $2.966278315-0.037643511 \quad 1.013725042$

$\begin{array}{llll}\text { C } & 3.989686251 & -0.259802192 & 0.076055393\end{array}$

$\begin{array}{llll}\mathrm{H} & 4.936488152 & 0.259179592 & 0.192367852\end{array}$

C $3.786818504-1.137598276-0.987068892$

H $\quad 4.584226131 \quad-1.299629688-1.707523346$

C $2.567569733-1.804763198-1.128966212$

H $\quad 2.414010763 \quad-2.491929293-1.956803918$

C $\quad 1.543233752-1.579242110-0.209360659$

$\begin{array}{lllll}\mathrm{H} & 0.588245749 & -2.085727930 & -0.320977747\end{array}$

C $\quad 1.739031672-0.696660638 \quad 0.855459332$

H $\quad 0.924676716 \quad-0.521554351 \quad 1.551612973$

C $\quad 6.710963249 \quad 2.479707718 \quad 2.085159063$

$\begin{array}{llll}\text { O } & 7.425552845 & 3.230016947 & 1.431923032\end{array}$

$\begin{array}{llll}\text { O } & 6.307159901 & 2.638784885 & 3.326630592\end{array}$

$\begin{array}{lllll}\text { O } & 6.298868656 & 1.266038656 & 1.655607224\end{array}$

$\begin{array}{llll}\text { O } & 4.968864441 & 1.176334143 & 5.048791885\end{array}$

\begin{tabular}{|c|c|c|}
\hline \multicolumn{3}{|l|}{ K } \\
\hline Mo & $5.531481743 \quad 0.534959316$ & 3.66315126 \\
\hline $\mathrm{P}$ & $7.968601227 \quad 0.188136980$ & 4.440404892 \\
\hline $\mathrm{P}$ & $3.387288332 \quad 1.089367151$ & 2.403239727 \\
\hline $\mathrm{N}$ & $\begin{array}{lll}4.345849991 & -1.169270039\end{array}$ & 4.215057373 \\
\hline $\mathrm{N}$ & $6.068117142-1.170814037$ & 11085 \\
\hline $\mathrm{C}$ & $3.519243002-3.493079662$ & 3.803 \\
\hline $\mathrm{H}$ & $2.466792345-3.205576420$ & $3.72^{\prime}$ \\
\hline $\mathrm{H}$ & $187-3.910$ & 8538 \\
\hline $\mathrm{H}$ & $0-4.2969$ & 9652 \\
\hline $\mathrm{C}$ & $56-2.3231$ & 3.5 \\
\hline $\mathrm{C}$ & $5.474502087-2.344$ & 3567 \\
\hline $\mathrm{C}$ & $5.844754696 \quad-3.5831477$ & 5277 \\
\hline $\mathrm{H}$ & $6.929152966-3.714544058$ & 5582 \\
\hline $\mathrm{H}$ & $5.477945328-3.516483307$ & 04325 \\
\hline $\mathrm{H}$ & $5.428871155-4.4865$ & 2.22 \\
\hline $\mathrm{C}$ & $7.158535004-1.054853678$ & 9919 \\
\hline $\mathrm{H}$ & $7.070211887-0.072948813$ & 78201 \\
\hline $\mathrm{H}$ & $7.040047169-1.804346919$ & 0.600224435 \\
\hline $\mathrm{C}$ & $8.555020332-1.211199522$ & 2.021205902 \\
\hline $\mathrm{H}$ & $8.605384827-2.149915457$ & 2.589142561 \\
\hline $\mathrm{H}$ & $9.278809547-1.300603271$ & 1.201502085 \\
\hline $\mathrm{C}$ & $8.992032051-0.035270181$ & 2.908927202 \\
\hline $\mathrm{H}$ & $\begin{array}{ll}8.901656151 & 0.908530295\end{array}$ & 2.363633871 \\
\hline $\mathrm{H}$ & $10.037786484-0.152088106$ & 3.213203430 \\
\hline $\mathrm{C}$ & $3.208640814-0.969763458$ & \\
\hline
\end{tabular}

H $\quad 3.513565063 \quad-0.232834741 \quad 5.873185635$

H $\quad 2.967227697 \quad-1.901932597 \quad 5.651575565$

C $\quad 1.946847796-0.474908054 \quad 4.390253067$

H $\quad 1.140188694 \quad-0.409039140 \quad 5.131589890$

H $\quad 1.638910532 \quad-1.233542919 \quad 3.664386511$

C $\quad 2.054606676 \quad 0.896344662 \quad 3.691037893$

H $\quad 2.293186426 \quad 1.673790812 \quad 4.424862385$

H $\quad 1.091955543 \quad 1.160021663 \quad 3.237735271$

C $\quad 8.379816055-1.275625348 \quad 5.462393761$

$\begin{array}{llll}\text { C } & 7.339019775 & -2.079585791 & 5.949663162\end{array}$

H $\quad 6.310281754-1.8068149095 .735629559$

$\begin{array}{llll}\text { C } & 7.622030735 & -3.226057529 & 6.694695950\end{array}$

H $\quad 6.807736874 \quad-3.841239452 \quad 7.068100929$

$\begin{array}{llll}\text { C } & 8.944693565 & -3.581285000 & 6.959108829\end{array}$

H $\quad 9.164369583 \quad-4.473373413 \quad 7.539368153$

$\begin{array}{llll}\text { C } & 9.988906860 & -2.787204027 & 6.477308273\end{array}$

H $11.020433426 \quad-3.059437275 \quad 6.683431625$

$\begin{array}{llll}\text { C } & 9.709216118 & -1.642500162 & 5.732839108\end{array}$

H $\quad 10.527321815 \quad-1.024379730 \quad 5.373371601$

$\begin{array}{llll}\text { C } & 8.721666336 & 1.647974849 & 5.239737034\end{array}$

$\begin{array}{llll}\text { C } & 8.715353012 & 2.863010883 & 4.528069019\end{array}$

H $\quad 8.279189110 \quad 2.934820890 \quad 3.533482552$

C $\quad 9.255115509 \quad 4.005704403 \quad 5.116971016$

H $\quad 9.251446724 \quad 4.937765598 \quad 4.558918953$

C $\quad 9.786475182 \quad 3.959211588 \quad 6.407866955$

H $\quad 10.203908920 \quad 4.855508327 \quad 6.858964920$

C $\quad 9.774137497 \quad 2.759766102 \quad 7.120372772$

H $\quad 10.177668571 \quad 2.717737913 \quad 8.128606796$

$\begin{array}{llll}\text { C } & 9.242130280 & 1.606764555 & 6.541664600\end{array}$

$\begin{array}{llll}\mathrm{H} & 9.235103607 & 0.680081785 & 7.106761456\end{array}$

$\begin{array}{llll}\text { C } & 3.145368338 & 2.770777225 & 1.748606682\end{array}$

$\begin{array}{llll}\text { C } & 2.153491259 & 3.040775537 & 0.793936968\end{array}$

$\begin{array}{llll}\mathrm{H} & 1.521783948 & 2.238167524 & 0.422501564\end{array}$

$\begin{array}{llll}\text { C } & 1.999540329 & 4.332807541 & 0.297114491\end{array}$

H $\quad 1.236885905 \quad 4.537226200 \quad-0.449428111$

$\begin{array}{llll}\text { C } & 2.838337898 & 5.357728958 & 0.743095577\end{array}$

$\begin{array}{llll}\mathrm{H} & 2.728981018 & 6.360638618 & 0.339466512\end{array}$

$\begin{array}{llll}\text { C } & 3.828860760 & 5.090844154 & 1.688294768\end{array}$

$\begin{array}{llll}\mathrm{H} & 4.504001141 & 5.876010418 & 2.013956308\end{array}$

C $3.9843852523 .801263809 \quad 2.195274353$

H $\quad 4.785717487 \quad 3.592712641 \quad 2.896368265$

$\begin{array}{llll}\text { C } & 2.982971907 & -0.054030370 & 1.034780860\end{array}$

$\begin{array}{llll}\text { C } & 3.967240334 & -0.234048411 & 0.046842493\end{array}$

$\begin{array}{lllll}\mathrm{H} & 4.878229618 & 0.362100899 & 0.086720355\end{array}$

C $3.750954390-1.141788602-0.988725066$

H $\quad 4.514595032-1.271371007-1.751181960$

C $\quad 2.563205242-1.875820279-1.052882791$

H $\quad 2.403985262-2.588284254-1.858162761$

C $\quad 1.574162245-1.680460334-0.088425227$ 
H $\quad 0.639961302 \quad-2.232929468 \quad-0.143273249$

C $\quad 1.779680490 \quad-0.768751204 \quad 0.949815869$

$\begin{array}{llll}\mathrm{H} & 0.991480172 & -0.617981970 & 1.680213690\end{array}$

$\begin{array}{llll}\text { C } & 6.665659904 & 2.468701839 & 1.272328734\end{array}$

O $\quad 7.286459446 \quad 3.507644653 \quad 1.484707952$

$\begin{array}{llll}\mathrm{O} & 6.539627552 & 1.648757339 & 2.506992579\end{array}$

$\begin{array}{lllll}\text { O } & 6.152370453 & 1.929911494 & 0.280949801\end{array}$

O $4.929512501 \quad 1.265809536 \quad 5.113446236$

\section{K-TS}

$\begin{array}{lllll}\text { Mo } & 5.568182468 & 0.523353577 & 3.609927177\end{array}$

$\begin{array}{llll}\mathrm{P} & 7.984806538 & 0.188683555 & 4.478140354\end{array}$

$\begin{array}{llll}\text { P } & 3.376076937 & 1.049737096 & 2.393974066\end{array}$

$\begin{array}{llll}\mathrm{N} & 4.395914555 & -1.206536770 & 4.174677849\end{array}$

N $\quad 6.123676777 \quad-1.177395105 \quad 2.342638016$

C $3.613525391 \quad-3.547450066 \quad 3.772017956$

$\mathrm{H} \quad 2.555406332 \quad-3.280601025 \quad 3.694815397$

H $\quad 3.772790909 \quad-3.952428102 \quad 4.779794216$

H $\quad 3.786653996-4.355933666 \quad 3.063194990$

$\begin{array}{llll}\text { C } & 4.509140968 & -2.361660480 & 3.501862288\end{array}$

$\begin{array}{llll}\text { C } & 5.543698788 & -2.365343809 & 2.529285669\end{array}$

C $\quad 5.936390400 \quad-3.590735197 \quad 1.738240480$

H $\quad 7.021266937 \quad-3.730100393 \quad 1.732529998$

H $\quad 5.608196259 \quad-3.505453587 \quad 0.694249630$

H $\quad 5.500129700 \quad-4.5003013612 .149457932$

C $7.236085415-1.058860421 \quad 1.388504863$

H $7.168831348 \quad-0.070870563 \quad 0.930258036$

H $\quad 7.137951851 \quad-1.799648523 \quad 0.589098275$

$\begin{array}{llll}\text { C } & 8.614914894 & -1.223856568 & 2.059747458\end{array}$

H $\quad 8.640626907 \quad-2.163526535 \quad 2.627247095$

H $\quad 9.363739967 \quad-1.317529678 \quad 1.262776375$

C $9.031469345-0.050318956 \quad 2.961543560$

$\begin{array}{lllll}\mathrm{H} & 8.936065674 & 0.894032061 & 2.415950537\end{array}$

$\begin{array}{llll}\text { H } & 10.076639175 & -0.154974401 & 3.271792889\end{array}$

C $3.259073496-1.028814077 \quad 5.092217922$

H $\quad 3.553258181 \quad-0.283743054 \quad 5.832565308$

H $\quad 3.036329985 \quad-1.964134812 \quad 5.617393970$

C $1.983093858-0.554307461 \quad 4.364426136$

H $\quad 1.174313426-0.521290958 \quad 5.105984688$

H $\quad 1.692706823 \quad-1.303403616 \quad 3.620673895$

C $\quad 2.065812588 \quad 0.833582103 \quad 3.697055101$

$\mathrm{H} \quad 2.323507071 \quad 1.592222691 \quad 4.444306850$

H $\quad 1.093081832 \quad 1.108233929 \quad 3.273044825$

$\begin{array}{llll}\text { C } & 8.340801239 & -1.264438033 & 5.535497665\end{array}$

C $7.264938831 \quad-1.937573075 \quad 6.134357452$

H $\quad 6.253438950 \quad-1.580395222 \quad 5.965855598$

C $7.488589764-3.064636946 \quad 6.927524567$ $\begin{array}{llll}\text { H } & 6.646219254 & -3.575264692 & 7.386447430\end{array}$

$\begin{array}{llll}\text { C } \quad 8.786737442 & -3.535876989 & 7.128372192\end{array}$

H $\quad 8.960309982-4.414390087 \quad 7.743615150$

$\begin{array}{llll}\text { C } & 9.864791870 & -2.873104811 & 6.534705639\end{array}$

H $\quad 10.877947807 \quad-3.234371901 \quad 6.688876629$

C $\quad 9.644457817-1.745407104 \quad 5.744195461$

H $\quad 10.492383003 \quad-1.232389092 \quad 5.298940182$

C $\quad 8.771636963 \quad 1.642654181 \quad 5.263339520$

$\begin{array}{llll}\text { C } & 8.718685150 & 2.862854958 & 4.562268734\end{array}$

$\begin{array}{llll}\mathrm{H} & 8.223770142 & 2.928086758 & 3.595738173\end{array}$

C $\quad 9.297191620 \quad 4.003752708 \quad 5.117017746$

$\begin{array}{llll}\mathrm{H} & 9.257666588 & 4.938813210 & 4.565222740\end{array}$

C $\quad 9.916370392 \quad 3.950743437 \quad 6.368765831$

H $\quad 10.363375664 \quad 4.844423294 \quad 6.795624256$

C $\quad 9.953593254 \quad 2.745603800 \quad 7.071260452$

$\begin{array}{llll}\mathrm{H} & 10.427038193 & 2.697053909 & 8.048369408\end{array}$

$\begin{array}{llll}\text { C } & 9.383712769 & 1.594402790 & 6.523612976\end{array}$

$\begin{array}{llll}\mathrm{H} & 9.419500351 & 0.662337601 & 7.079270840\end{array}$

C $\quad 3.108968496 \quad 2.742296696 \quad 1.771020055$

C $\quad 2.176723003 \quad 3.026931763 \quad 0.763749003$

$\begin{array}{llll}\mathrm{H} & 1.595609188 & 2.224650383 & 0.317265272\end{array}$

$\begin{array}{llll}\text { C } & 2.015543938 & 4.336309910 & 0.313679248\end{array}$

H $\quad 1.299111843 \quad 4.552054405 \quad-0.474354118$

$\begin{array}{llll}\text { C } & 2.784073114 & 5.365251064 & 0.862863660\end{array}$

$\begin{array}{llll}\mathrm{H} & 2.666298151 & 6.382731438 & 0.500084341\end{array}$

$\begin{array}{llll}\text { C } & 3.714916229 & 5.084929943 & 1.864260674\end{array}$

H $\quad 4.330627441 \quad 5.878292084 \quad 2.277534246$

$\begin{array}{llll}\text { C } & 3.881288290 & 3.777422190 & 2.318601131\end{array}$

H $\quad 4.633330345 \quad 3.5550193793 .069499016$

C $\quad 2.948398352-0.080299601 \quad 1.018216491$

$\begin{array}{llll}\text { C } & 3.934542418 & -0.315294355 & 0.044556163\end{array}$

$\begin{array}{llll}\mathrm{H} & 4.872732639 & 0.227962300 & 0.095284417\end{array}$

C $3.689975500-1.208048463-0.998101771$

H $\quad 4.458185673-1.374562144-1.748618960$

C $2.467945337-1.881196618-1.083537579$

H $\quad 2.284183264 \quad-2.582180262-1.893238187$

C $\quad 1.478177786-1.637400270-0.130489558$

H $\quad 0.518767834-2.143401623 \quad-0.197373375$

$\begin{array}{llll}\text { C } & 1.714228749 & -0.738467216 & 0.912621617\end{array}$

H $\quad 0.926253378 \quad-0.554392576 \quad 1.636095166$

$\begin{array}{llll}\text { C } & 6.636265278 & 2.594201326 & 1.055026412\end{array}$

$\begin{array}{llll}\text { O } & 7.373203754 & 3.505760431 & 1.334056735\end{array}$

$\begin{array}{llll}\text { O } & 6.517163754 & 1.601058245 & 2.445442915\end{array}$

$\begin{array}{llll}\text { O } & 5.971681118 & 2.089360714 & 0.176650181\end{array}$

$\begin{array}{llll}\text { O } & 4.933842182 & 1.245250106 & 5.064011574\end{array}$

L 


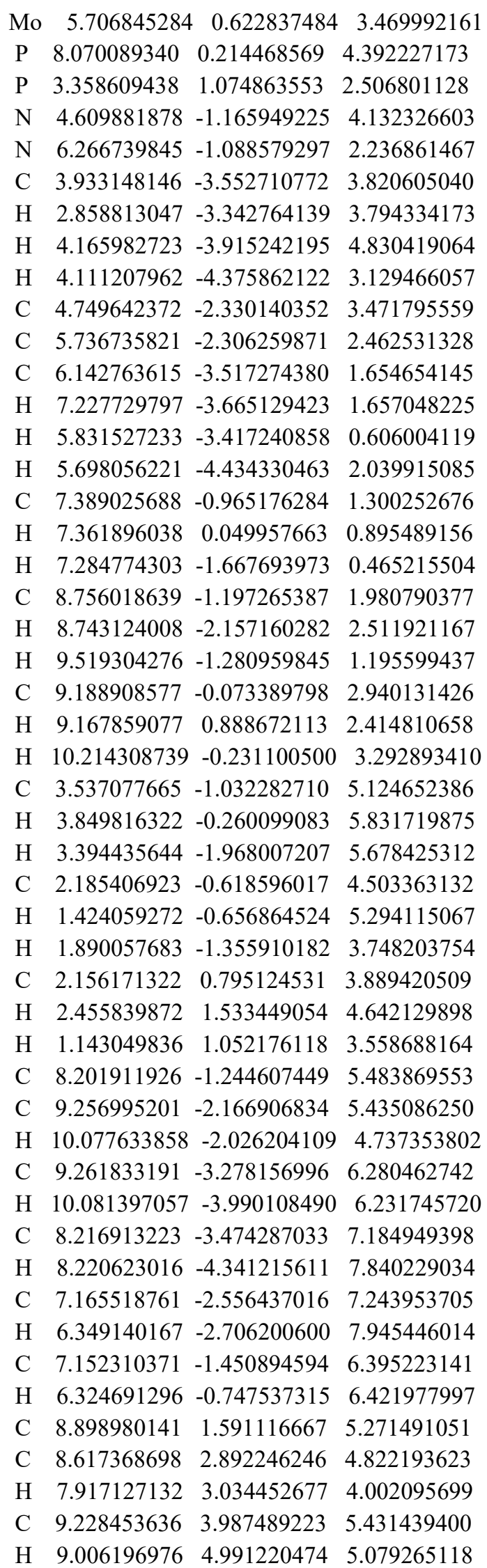

$\begin{array}{llll}\text { C } & 10.108858109 & 3.799039602 & 6.499887466\end{array}$

$\begin{array}{llll}\mathrm{H} & 10.575311661 & 4.655498028 & 6.978935719\end{array}$

$\begin{array}{llll}\text { C } & 10.381649017 & 2.507927895 & 6.956175804\end{array}$

H $11.060157776 \quad 2.356918097 \quad 7.791653633$

$\begin{array}{llll}\text { C } & 9.781844139 & 1.405635238 & 6.344265938\end{array}$

H $\quad 9.993057251 \quad 0.403825492 \quad 6.707293510$

$\begin{array}{llll}\text { C } & 2.921623230 & 2.730969429 & 1.856461883\end{array}$

C $\quad 1.651436687 \quad 3.007142067 \quad 1.326919079$

$\begin{array}{llll}\mathrm{H} & 0.902460337 & 2.220694542 & 1.272988200\end{array}$

$\begin{array}{llll}\text { C } & 1.352132559 & 4.280373573 & 0.847637177\end{array}$

$\begin{array}{llll}\mathrm{H} & 0.367618471 & 4.488081932 & 0.436798781\end{array}$

$\begin{array}{llll}\text { C } & 2.322066545 & 5.286428452 & 0.888173223\end{array}$

$\begin{array}{llll}\mathrm{H} & 2.089176416 & 6.278716564 & 0.510858893\end{array}$

C $\quad 3.589959621 \quad 5.014063358 \quad 1.402171016$

H $\quad 4.348379135 \quad 5.791932583 \quad 1.421649933$

$\begin{array}{llll}\text { C } & 3.895924330 & 3.739215851 & 1.884383678\end{array}$

H $\quad 4.890601635 \quad 3.511986732 \quad 2.257082224$

$\begin{array}{llll}\text { C } & 2.872889519 & -0.069438584 & 1.159238338\end{array}$

$\begin{array}{llll}\text { C } & 3.844842434 & -0.359848648 & 0.187560156\end{array}$

$\begin{array}{llll}\mathrm{H} & 4.831517220 & 0.085578397 & 0.272274315\end{array}$

C $\quad 3.553817034-1.224758029-0.865778863$

H $\quad 4.314334393 \quad-1.440464020-1.611671567$

C $2.292689323-1.819030166-0.958369195$

H $\quad 2.069269657 \quad-2.500192881 \quad-1.775086522$

C $\quad 1.320420980-1.535128593 \quad 0.001310660$

H $\quad 0.336844444-1.992383838-0.067373551$

C $\quad 1.607526898 \quad-0.663053453 \quad 1.053432703$

H $\quad 0.838035822-0.451285720 \quad 1.790155768$

$\begin{array}{lllll}\text { O } & 6.498795033 & 1.665574074 & 2.266518593\end{array}$

$\begin{array}{llll}\text { O } & 5.154212952 & 1.346249938 & 4.985466957\end{array}$ 
C $\quad 1.797674894-3.520335913-3.046123981$

$\begin{array}{lllll}\text { H } & 1.901393175 & -2.548690796 & -3.540445328\end{array}$

H $\quad 1.490655303 \quad-4.247105122-3.806295633$

C $3.151386499-3.954063892-2.448749065$

H $\quad 3.034788847 \quad-4.919889927-1.940747857$

H $\quad 3.844141006-4.116277695 \quad-3.284236670$

C $3.798837423 \quad-2.932567120-1.496492267$

H $3.963685989-1.983732104-2.011445999$

H $\quad 4.780283928$-3.282565594 -1.157552242

$\begin{array}{lllll}\text { C } & -1.760674596 & -2.467593908 & 1.009609818\end{array}$

$\mathrm{H} \quad-1.176754594 \quad-1.929575086 \quad 1.760532141$

$\begin{array}{llll}\mathrm{H} & -2.236905336 & -3.323720455 & 1.497667193\end{array}$

$\begin{array}{llll}\text { C } & -2.807979107 & -1.509556890 & 0.412483990\end{array}$

H $\quad-3.393866539-2.031434536-0.351771891$

$\begin{array}{llll}\text { H } & -2.271603823 & -0.710078537 & -0.101899616\end{array}$

$\begin{array}{llll}\text { C } & -3.727771044 & -0.900012851 & 1.474243402\end{array}$

$\begin{array}{llll}\mathrm{H} & -3.120613098 & -0.482906133 & 2.286848307\end{array}$

H $\quad-4.407808781 \quad-1.649594426 \quad 1.898907661$

$\begin{array}{llll}\text { C } & 2.651955128 & -3.964879274 & 1.005783916\end{array}$

C $\quad 1.652335882-4.000889778 \quad 1.991005778$

$\begin{array}{llll}\mathrm{H} & 0.988241255 & -3.149155855 & 2.107498646\end{array}$

C $\quad 1.507084131 \quad-5.118566513 \quad 2.810389996$

$\begin{array}{llll}\mathrm{H} & 0.731880665 & -5.131437302 & 3.572076797\end{array}$

C $\quad 2.352322578 \quad-6.219789982 \quad 2.651690006$

H $\quad 2.235415697 \quad-7.093646526 \quad 3.286844730$

C $3.348721504-6.193590164 \quad 1.675969362$

$\begin{array}{llll}\mathrm{H} & 4.011602879 & -7.045511723 & 1.549480557\end{array}$

C $3.500322580-5.071752548 \quad 0.859370410$

H $\quad 4.285151482 \quad-5.067245960 \quad 0.108932100$

C $3.907966852-1.365021110 \quad 0.936351657$

C $5.124820709-1.841949582 \quad 1.445165396$

$\begin{array}{llll}\text { H } & 5.392156124 & -2.889756918 & 1.332114697\end{array}$

C $\quad 5.998433590 \quad-0.975822985 \quad 2.097985268$

H $\quad 6.947505474-1.346832514 \quad 2.475054502$

$\begin{array}{llll}\text { C } & 5.656849861 & 0.370133430 & 2.262755156\end{array}$

$\begin{array}{llll}\mathrm{H} & 6.344157219 & 1.044289947 & 2.767520189\end{array}$

C $\quad 4.439231873 \quad 0.843515158 \quad 1.773885846$

$\begin{array}{llll}\mathrm{H} & 4.160420418 & 1.887175918 & 1.894223928\end{array}$

C $\quad 3.566408873-0.018228021 \quad 1.107349157$

$\begin{array}{llll}\mathrm{H} & 2.637520790 & 0.370814711 & 0.711135983\end{array}$

$\begin{array}{llll}\text { C } & -6.023411274 & 0.756240368 & 2.029685259\end{array}$

C $\quad-7.218515873 \quad 1.411153436 \quad 1.679299116$

$\begin{array}{llll}\mathrm{H} & -7.393522739 & 1.684976220 & 0.641651213\end{array}$

$\begin{array}{llll}\text { C } & -8.189777374 & 1.698632598 & 2.637031555\end{array}$

$\begin{array}{llll}\mathrm{H} & -9.107988358 & 2.197416782 & 2.337320089\end{array}$

$\begin{array}{llll}\text { C } & -7.987432003 & 1.350813389 & 3.975401163\end{array}$

H $\quad-8.742632866 \quad 1.577971816 \quad 4.722627163$

C $-6.804669380 \quad 0.707676232 \quad 4.340606213$

$\begin{array}{llll}\mathrm{H} & -6.633568287 & 0.429427713 & 5.377753258\end{array}$
C $\quad-5.834615231 \quad 0.411605209 \quad 3.379660606$

H $\quad-4.921982765 \quad-0.084868699 \quad 3.694119692$

C $-5.575353622-0.277022123-0.641072810$

C $\quad-4.986610889-0.129223838-1.907639861$

$\begin{array}{lllll}\mathrm{H} & -4.096769810 & 0.478716791 & -2.040419579\end{array}$

C $\quad-5.515901089-0.783886790-3.021452188$

$\begin{array}{llll}\mathrm{H} & -5.018902302 & -0.670683861 & -3.980580807\end{array}$

C $-6.660695076-1.571483731-2.890065908$

H $\quad-7.081631660-2.075473070-3.756310701$

C $-7.264583588-1.712006450-1.635924935$

H $\quad-8.156723022-2.324551344-1.527458906$

C $-6.722247124-1.078399539-0.517242849$

$\begin{array}{llll}\mathrm{H} & -7.193377495 & -1.202538490 & 0.454393297\end{array}$

$\begin{array}{lllll}\text { O } & 0.343170911 & -0.595851243 & 0.677914262\end{array}$

$\begin{array}{lllll}\text { O } & -0.911740124 & -0.870197833 & -2.138789892\end{array}$

$\begin{array}{llll}\text { C } & -1.363845944 & 0.333496809 & -2.405428648\end{array}$

$\begin{array}{lllll}\text { O } & -2.160325766 & 0.579207003 & -3.307223082\end{array}$

$\begin{array}{llll}\text { O } & -0.985111237 & 1.304848552 & -1.532237291\end{array}$

$\begin{array}{llll}\text { O } & 1.870270729 & -0.499482960 & -2.039360046\end{array}$

P $\quad-1.040548563 \quad 3.712017536 \quad 0.017429462$

Mo $\quad 0.658331275 \quad 2.474574566-1.477093697$

N $\quad 2.151936293 \quad 3.909206629 \quad-2.136591911$

$\begin{array}{lllll}\mathrm{N} & -0.257775247 & 3.885312319 & -3.022700071\end{array}$

$\begin{array}{lllll}\text { O } & 1.300142765 & 1.392976642 & -3.135697126\end{array}$

C $-2.645011902 \quad 3.830348492-0.902702332$

$\begin{array}{llll}\text { C } & -0.683064878 & 5.455697536 & 0.482734591\end{array}$

$\begin{array}{llll}\text { C } & -1.468639255 & 2.861886740 & 1.592504859\end{array}$

$\begin{array}{lllll}\text { P } & 6.146997929 & 0.488909721 & -1.532545447\end{array}$

C $\quad 5.578263283 \quad 2.280608416-1.411597848$

$\begin{array}{llll}\text { C } & 7.940558910 & 0.635569036 & -1.119803429\end{array}$

$\begin{array}{llll}\text { C } & 6.153412342 & 0.267654181 & -3.372456074\end{array}$

C $\quad 1.929661393 \quad 4.678843498$-3.189776659

C $3.5219466693 .775767326-1.596195936$

C $\quad 0.539878070 \quad 4.755281925 \quad-3.598289013$

$\begin{array}{lllll}\text { C } & -1.695378661 & 3.834367514 & -3.325966358\end{array}$

C $\quad 3.021602631 \quad 5.429914474$-3.915477991

H $3.901870012 \quad 4.795742512-4.050771713$

H $\quad 3.340385914 \quad 6.330040932-3.372859001$

H $\quad 2.695849180 \quad 5.740168095 \quad-4.909264565$

C $\quad 0.058920186 \quad 5.793921947-4.586898804$

H $\quad-0.877242982 \quad 6.245687008 \quad-4.248167515$

$\mathrm{H} \quad-0.127012223 \quad 5.348523140-5.572168350$

H $\quad 0.782765985 \quad 6.599639893 \quad-4.714638233$

H $\quad-1.979133368 \quad 2.777106524 \quad-3.381709814$

H $\quad-1.897726297 \quad 4.284012318-4.303151608$

C $\quad-2.551620483 \quad 4.549402237 \quad-2.260184765$

$\mathrm{H} \quad-2.187993288 \quad 5.576426983 \quad-2.118838549$

H $\quad-3.569758177 \quad 4.628570080-2.661453724$

H $\quad-2.977269173 \quad 2.795676470-1.029783368$ 


\begin{tabular}{|c|c|c|c|c|c|c|c|}
\hline $\mathrm{H}$ & -3.372298717 & 4.345688343 & -0.266972929 & $\mathrm{C}$ & 1.620362639 & 0.120320141 & -3.223356485 \\
\hline $\mathrm{H}$ & 3.424412489 & 3.751457691 & -0.507516146 & $\mathrm{O}$ & 1.790123224 & -0.463287383 & -4.288746357 \\
\hline $\mathrm{H}$ & 4.133128643 & 4.645183086 & -1.860276937 & & & & \\
\hline $\mathrm{C}$ & 4.200194836 & 2.472930908 & -2.060626984 & & & & \\
\hline $\mathrm{H}$ & 4.285678387 & 2.464896679 & -3.152027130 & $\mathbf{N}$ & & & \\
\hline $\mathrm{H}$ & 3.549224138 & 1.637689114 & -1.794938087 & & & & \\
\hline $\mathrm{H}$ & 5.508557320 & 2.494934559 & -0.337844312 & Mo & 0.628100693 & $3-1.611706138$ & $8-0.519386888$ \\
\hline $\mathrm{H}$ & 6.323176384 & 2.958227158 & -1.846159935 & $\mathrm{P}$ & 2.969220877 & -2.381241798 & -0.040983897 \\
\hline $\mathrm{C}$ & 0.425761372 & 6.095402241 & -0.088905111 & $\mathrm{P}$ & -4.980401516 & 0.655630827 & 0.416932851 \\
\hline $\mathrm{C}$ & -1.510911703 & 6.181186199 & 1.354107976 & $\mathrm{~N}$ & -1.061222434 & -2.870655060 & 0.456397772 \\
\hline $\mathrm{H}$ & 1.075013041 & 5.537529469 & -0.754812419 & $\mathrm{~N}$ & 0.678895593 & -3.615210295 & -1.247157216 \\
\hline $\mathrm{C}$ & 0.695200920 & 7.435274124 & 0.196004197 & $\mathrm{C}$ & -1.986738205 & -5.151852131 & 0.879096210 \\
\hline $\mathrm{H}$ & 1.559005380 & 7.918145657 & -0.253871262 & $\mathrm{H}$ & -3.027591228 & -4.949423790 & 0.602570951 \\
\hline $\mathrm{C}$ & -0.136631221 & 8.149580002 & 1.057481885 & $\mathrm{H}$ & -1.919028759 & -5.091182709 & 1.970600247 \\
\hline $\mathrm{H}$ & 0.073530793 & 9.192099571 & 1.281081319 & $\mathrm{H}$ & -1.766500473 & -6.179526329 & 0.591571748 \\
\hline $\mathrm{C}$ & -1.239326596 & 7.517983437 & 1.638268709 & $\mathrm{C}$ & -1.042974472 & -4.168886185 & 0.223244429 \\
\hline $\mathrm{H}$ & -1.886918902 & 8.066953659 & 2.316701174 & $\mathrm{C}$ & -0.092038244 & -4.587414742 & -0.785076737 \\
\hline $\mathrm{H}$ & -2.362257004 & 5.700937748 & 1.826020718 & $\mathrm{C}$ & -0.045054611 & -6.002311230 & -1.304822922 \\
\hline $\mathrm{C}$ & -2.700805902 & 3.049754143 & 2.242496729 & $\mathrm{H}$ & 0.877214432 & -6.200772285 & -1.851092100 \\
\hline $\mathrm{C}$ & -0.532677472 & 1.986126184 & 2.162616491 & $\mathrm{H}$ & -0.891433954 & -6.222002506 & -1.967721105 \\
\hline $\mathrm{H}$ & -3.469702244 & 3.677569389 & 1.804903269 & $\mathrm{H}$ & -0.084852979 & -6.713937759 & -0.475397021 \\
\hline $\mathrm{C}$ & -2.975750923 & 2.404906034 & 3.448302746 & $\mathrm{C}$ & 1.453248620 & -3.767658472 & -2.508960247 \\
\hline $\mathrm{H}$ & -3.939161062 & 2.552865267 & 3.927270889 & $\mathrm{H}$ & 1.366653562 & -2.813649654 & -3.047981024 \\
\hline $\mathrm{C}$ & -2.032768250 & 1.540896416 & 4.012365341 & $\mathrm{H}$ & 0.992979646 & -4.537532330 & -3.134073973 \\
\hline $\mathrm{H}$ & -2.251867056 & 1.031116843 & 4.947141171 & $\mathrm{C}$ & 2.949933767 & -4.057625771 & -2.299695969 \\
\hline $\mathrm{C}$ & -0.816101909 & 1.329694986 & 3.362432718 & $\mathrm{H}$ & 3.074511290 & -4.974592686 & -1.710672021 \\
\hline $\mathrm{H}$ & -0.081490621 & 0.643813312 & 3.774921179 & $\mathrm{H}$ & 3.380120754 & -4.249985695 & -3.290141106 \\
\hline $\mathrm{H}$ & 0.406131476 & 1.790920138 & 1.659416795 & $\mathrm{C}$ & 3.713614941 & -2.883788347 & -1.660124660 \\
\hline $\mathrm{C}$ & 8.814881325 & -0.398832470 & -1.501701236 & $\mathrm{H}$ & 3.627135277 & -1.995822430 & -2.294339418 \\
\hline $\mathrm{C}$ & 8.458076477 & 1.671472907 & -0.324723810 & $\mathrm{H}$ & 4.779274464 & -3.108943939 & -1.550319672 \\
\hline $\mathrm{H}$ & 8.442127228 & -1.212943196 & -2.118865967 & $\mathrm{C}$ & -2.093220234 & -2.279842854 & 1.322106481 \\
\hline $\mathrm{C}$ & 10.156286240 & -0.385723740 & -1.123622179 & $\mathrm{H}$ & -1.605447054 & -1.856554866 & 2.208598137 \\
\hline $\mathrm{H}$ & 10.812501907 & -1.191009283 & $3-1.444237828$ & $\mathrm{H}$ & -2.799572945 & -3.037078619 & 1.675239086 \\
\hline $\mathrm{C}$ & 10.658036232 & 0.654895008 & -0.336976707 & $\mathrm{C}$ & -2.859267235 & -1.173461556 & 0.577634811 \\
\hline $\mathrm{H}$ & 11.703477859 & 0.665851057 & -0.041401654 & $\mathrm{H}$ & -3.163135052 & -1.557195544 & -0.400830448 \\
\hline $\mathrm{C}$ & 9.800484657 & 1.679915786 & 0.062130701 & $\mathrm{H}$ & -2.185703993 & -0.333924860 & 0.393870205 \\
\hline $\mathrm{H}$ & 10.176468849 & 2.496321201 & 0.674003243 & $\mathrm{C}$ & -4.073478699 & -0.682831943 & 1.372223973 \\
\hline $\mathrm{H}$ & 7.816597939 & 2.485301495 & -0.000970306 & $\mathrm{H}$ & -3.738510132 & -0.235256195 & 2.314311743 \\
\hline $\mathrm{C}$ & 4.949026108 & -0.190608904 & -3.930720568 & $\mathrm{H}$ & -4.763209343 & -1.505321145 & 1.605601788 \\
\hline $\mathrm{C}$ & 7.215379715 & 0.576848567 & -4.237396717 & $\mathrm{C}$ & 3.127717733 & -3.906777620 & 0.991357923 \\
\hline $\mathrm{H}$ & 4.104432583 & -0.419687480 & -3.291783571 & $\mathrm{C}$ & 2.154899836 & -4.205897808 & 1.957402587 \\
\hline $\mathrm{C}$ & 4.791097641 & -0.320775002 & -5.310239315 & $\mathrm{H}$ & 1.313887715 & -3.538161516 & 2.100136995 \\
\hline $\mathrm{H}$ & 3.824047089 & -0.632427633 & -5.691642284 & $\mathrm{C}$ & 2.252167940 & -5.359082699 & 2.736387730 \\
\hline $\mathrm{C}$ & 5.859140396 & -0.024476508 & -6.158781052 & $\mathrm{H}$ & 1.489458799 & -5.567577839 & 3.482330084 \\
\hline $\mathrm{H}$ & 5.749545097 & -0.130431101 & -7.235118389 & $\mathrm{C}$ & 3.318954468 & -6.240458488 & 2.559422731 \\
\hline $\mathrm{C}$ & 7.070509434 & 0.418712556 & -5.617526054 & $\mathrm{H}$ & 3.392702341 & -7.140330791 & 3.164310455 \\
\hline $\mathrm{H}$ & 7.904140949 & 0.657354355 & -6.274369717 & $\mathrm{C}$ & 4.289201736 & -5.961172581 & 1.595984578 \\
\hline $\mathrm{H}$ & 8.157629967 & 0.937763214 & -3.834366798 & $\mathrm{H}$ & 5.121527672 & -6.643546104 & 1.444674015 \\
\hline $\mathrm{O}$ & 1.714109182 & 2.169358253 & -0.164564371 & $\mathrm{C}$ & 4.192858696 & -4.806680202 & 0.819219947 \\
\hline
\end{tabular}


$\begin{array}{lllll}\mathrm{H} & 4.951082230 & -4.621270657 & 0.064562574\end{array}$

C $\quad 4.167704582-1.203241110 \quad 0.709721446$

$\begin{array}{llll}\text { C } & 5.505175591 & -1.570977449 & 0.927967072\end{array}$

$\begin{array}{llll}\mathrm{H} & 5.853549004 & -2.562316418 & 0.655029416\end{array}$

C $\quad 6.408853531 \quad-0.669727325 \quad 1.488688588$

$\begin{array}{llll}\text { H } & 7.445893764 & -0.963074267 & 1.625817060\end{array}$

C $\quad 5.985610008 \quad 0.611644447 \quad 1.854762673$

$\begin{array}{lllll}\mathrm{H} & 6.689964771 & 1.313048840 & 2.294060946\end{array}$

C $\quad 4.656734943 \quad 0.983752787 \quad 1.647246957$

$\begin{array}{lllll}\mathrm{H} & 4.306179047 & 1.974914670 & 1.916669130\end{array}$

C $\quad 3.755419970 \quad 0.086298123 \quad 1.070756316$

$\begin{array}{lllll}\mathrm{H} & 2.738570452 & 0.405681312 & 0.883277416\end{array}$

$\begin{array}{lllll}\text { C } & -6.424229145 & 0.996388376 & 1.518681884\end{array}$

C $\quad-7.473715305 \quad 1.777313113 \quad 0.999075353$

H $\quad-7.446893215 \quad 2.080065966-0.045507971$

C $\quad-8.551884651 \quad 2.159082890 \quad 1.794459820$

$\begin{array}{lllll}\mathrm{H} & -9.352614403 & 2.756033897 & 1.365064859\end{array}$

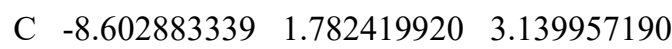

$\begin{array}{llll}\mathrm{H} & -9.438897133 & 2.085621834 & 3.764145851\end{array}$

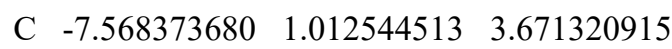

$\begin{array}{llll}\mathrm{H} & -7.597601414 & 0.708315253 & 4.714886189\end{array}$

$\begin{array}{llll}\text { C } & -6.492249966 & 0.619953513 & 2.869646549\end{array}$

$\begin{array}{lllll}\mathrm{H} & -5.705610275 & 0.015633883 & 3.309914589\end{array}$

C $\quad-5.740206718-0.366590023-0.927668095$

$\begin{array}{llll}\text { C } & -5.047032833 & -0.425573379 & -2.149197102\end{array}$

H $\quad-4.1230196950 .130501166-2.292904139$

$\begin{array}{llll}\text { C } & -5.526698589 & -1.217896461 & -3.195155144\end{array}$

H $\quad-4.968560696-1.258042216-4.126802444$

C $-6.710478783-1.942376494-3.046988726$

H $\quad-7.088291645 \quad-2.549580812-3.865759373$

C $-7.410631657-1.880175114-1.838321209$

$\begin{array}{lllll}\text { H } & -8.334560394 & -2.440647364 & -1.714540362\end{array}$

$\begin{array}{llll}\text { C } & -6.926898003 & -1.103819132 & -0.784456313\end{array}$

$\begin{array}{llll}\text { H } & -7.478411674 & -1.065147638 & 0.151370063\end{array}$

C $\quad 0.735013902-1.267908573 \quad 1.371656775$

$\begin{array}{lllll}\text { O } & -0.954996467 & -0.924820185 & -1.845527887\end{array}$

C $\quad-1.277800918 \quad 0.333322704-1.949438691$

$\begin{array}{lllll}\text { O } & -2.069060326 & 0.800183594 & -2.778603792\end{array}$

$\begin{array}{lllll}\text { O } & -0.713081181 & 1.128088713 & -1.005722404\end{array}$

$\begin{array}{llll}\text { O } & 1.794519067 & -0.162575543 & -1.394424319\end{array}$

P $-1.233555317 \quad 3.640893936-0.040571190$

$\begin{array}{lllll}\text { Mo } & 0.609400988 & 2.626658201 & -1.425509930\end{array}$

N $\quad 2.385546446 \quad 3.892118692-1.933282495$

$\begin{array}{lllll}\mathrm{N} & -0.007507190 & 4.206845284 & -2.597280502\end{array}$

O $\quad 1.368967295 \quad 1.386923432-2.947070122$

C $\quad-2.746037960 \quad 3.697962523-1.101691484$

$\begin{array}{llll}\text { C } & -1.006396532 & 5.402946472 & 0.465943873\end{array}$

$\begin{array}{llll}\text { C } & -1.778735399 & 2.787611723 & 1.499527454\end{array}$

$\begin{array}{lllll}\text { P } & 6.122130394 & 0.138888314 & -2.274907589\end{array}$
C $\quad 5.656222820 \quad 1.868821144 \quad-1.714265823$

C $\quad 7.903183460 \quad 0.036672894 \quad-1.786087394$

C $\quad 6.229783058 \quad 0.417432994-4.104955196$

C $\quad 2.144319057 \quad 5.038199425-2.560271502$

C $\quad 3.775958300 \quad 3.561913729-1.570536256$

C $\quad 0.7921120525 .186088562-3.003861427$

$\begin{array}{llll}\text { C } & -1.328941345 & 3.975269079 & -3.215332270\end{array}$

C $\quad 3.205537796 \quad 6.078327656-2.838320494$

H $\quad 3.9553880695 .713538647 \quad-3.550893784$

H $\quad 3.733220577 \quad 6.358335972-1.919477344$

H $\quad 2.774024725 \quad 6.987915516 \quad-3.256840706$

C $\quad 0.313791573 \quad 6.279536247 \quad-3.924135447$

$\begin{array}{lllll}\mathrm{H} & -0.734242558 & 6.532660961 & -3.722518921\end{array}$

H $\quad 0.394589245 \quad 5.978445530 \quad-4.982589722$

H $\quad 0.902498305 \quad 7.196121693 \quad-3.797830105$

H $-1.4576666362 .887609243 \quad-3.319763422$

H $\quad-1.343172550 \quad 4.402223587-4.222255707$

C $\quad-2.504544973 \quad 4.513772964-2.380930662$

H $\quad-2.344721079 \quad 5.572824001-2.139768600$

H $\quad-3.4043662554 .457077503 \quad-3.005236387$

$\mathrm{H} \quad-2.9575734142 .654119015-1.355218530$

H $\quad-3.6014161114 .092366695 \quad-0.545291960$

H $3.894563675 \quad 3.671696424-0.484316528$

H $\quad 4.473178387 \quad 4.264852047 \quad-2.034565449$

C $\quad 4.172399044 \quad 2.139700651 \quad-1.988342047$

H $\quad 3.942470551 \quad 2.011414766-3.050482512$

H $\quad 3.570643425 \quad 1.414494753 \quad-1.438914299$

H $\quad 5.846307278 \quad 1.915601373-0.636134863$

$\begin{array}{llll}\mathrm{H} & 6.297086239 & 2.609761238 & -2.210237503\end{array}$

$\begin{array}{llll}\text { C } & 0.253000140 & 6.005867004 & 0.333215117\end{array}$

$\begin{array}{llll}\text { C } & -2.071079016 & 6.180411816 & 0.950887144\end{array}$

H $\quad 1.084326506 \quad 5.426527500 \quad-0.051346194$

C $\quad 0.446548343 \quad 7.341721535 \quad 0.687381148$

$\begin{array}{llll}\mathrm{H} & 1.432107925 & 7.788055897 & 0.581445515\end{array}$

$\begin{array}{llll}\text { C } & -0.616957664 & 8.099199295 & 1.176723123\end{array}$

H $\quad-0.466789365 \quad 9.139113426 \quad 1.454610825$

C $-1.878654242 \quad 7.514305592 \quad 1.305988193$

$\begin{array}{llll}\mathrm{H} & -2.714349270 & 8.097138405 & 1.684474468\end{array}$

H $\quad-3.060743809 \quad 5.749033928 \quad 1.059126616$

$\begin{array}{llll}\text { C } & -3.014422178 & 3.079805613 & 2.102263212\end{array}$

$\begin{array}{llll}\text { C } & -0.941948473 & 1.849572062 & 2.119507313\end{array}$

H $\quad-3.714890003 \quad 3.757601023 \quad 1.627706528$

C $\quad-3.383413792 \quad 2.485170603 \quad 3.308590889$

$\begin{array}{llll}\mathrm{H} & -4.351072788 & 2.716590405 & 3.744841576\end{array}$

$\begin{array}{llll}\text { C } & -2.525331259 & 1.575313091 & 3.933034897\end{array}$

$\begin{array}{llll}\mathrm{H} & -2.809015036 & 1.113340259 & 4.875101089\end{array}$

C $-1.309441566 \quad 1.253695846 \quad 3.328011036$

$\begin{array}{llll}\mathrm{H} & -0.640755892 & 0.527410030 & 3.777916431\end{array}$

H $\quad-0.005372775 \quad 1.563417435 \quad 1.659643769$ 


$\begin{array}{lccc}\text { C } & 8.579878807 & -1.160621524 & -2.083623171 \\ \text { C } & 8.600817680 & 1.026183724 & -1.075826883 \\ \text { H } & 8.059072495 & -1.946027398 & -2.628115177 \\ \text { C } & 9.904029846 & -1.358329892 & -1.696911097 \\ \text { H } & 10.405011177 & -2.290992260 & -1.944672227 \\ \text { C } & 10.583671570 & -0.365649134 & -0.984842598 \\ \text { H } & 11.613294601 & -0.520300269 & -0.673947811 \\ \text { C } & 9.925153732 & 0.824861884 & -0.676445544 \\ \text { H } & 10.443144798 & 1.605686069 & -0.124573424 \\ \text { H } & 8.116888046 & 1.964491010 & -0.824671805 \\ \text { C } & 5.065621376 & 0.176909804 & -4.856855392 \\ \text { C } & 7.382647514 & 0.868844986 & -4.767600060 \\ \text { H } & 4.156835079 & -0.178881183 & -4.375071526\end{array}$

C $\quad 5.055955887 \quad 0.404027998 \quad-6.235500336$

H $\quad 4.142914295 \quad 0.222330645-6.796490192$

$\begin{array}{lllll}\text { C } & 6.207762718 & 0.849244714 & -6.886580467\end{array}$

H $\quad 6.201580048 \quad 1.014880061 \quad-7.961008072$

C $\quad 7.371774673 \quad 1.078308940-6.147613525$

H $\quad 8.274385452 \quad 1.423350215 \quad-6.646896839$

$\begin{array}{llll}\mathrm{H} & 8.292893410 & 1.054522514 & -4.203535557\end{array}$

$\begin{array}{llll}\text { C } & 1.570715547 & 2.942649364 & 0.212102666\end{array}$

C $\quad 1.7524937390 .164265186 \quad-2.715191841$

O $2.111666203-0.642288148-3.580428123$

$\begin{array}{llll}\text { O } & 2.184726238 & 3.086001635 & 1.205119252\end{array}$

$\begin{array}{llll}\text { O } & 0.785118997 & -1.024972081 & 2.523531675\end{array}$

Table S7. Vibrational frequencies (in $\mathrm{cm}^{-1}$ ) for the optimized geometries.

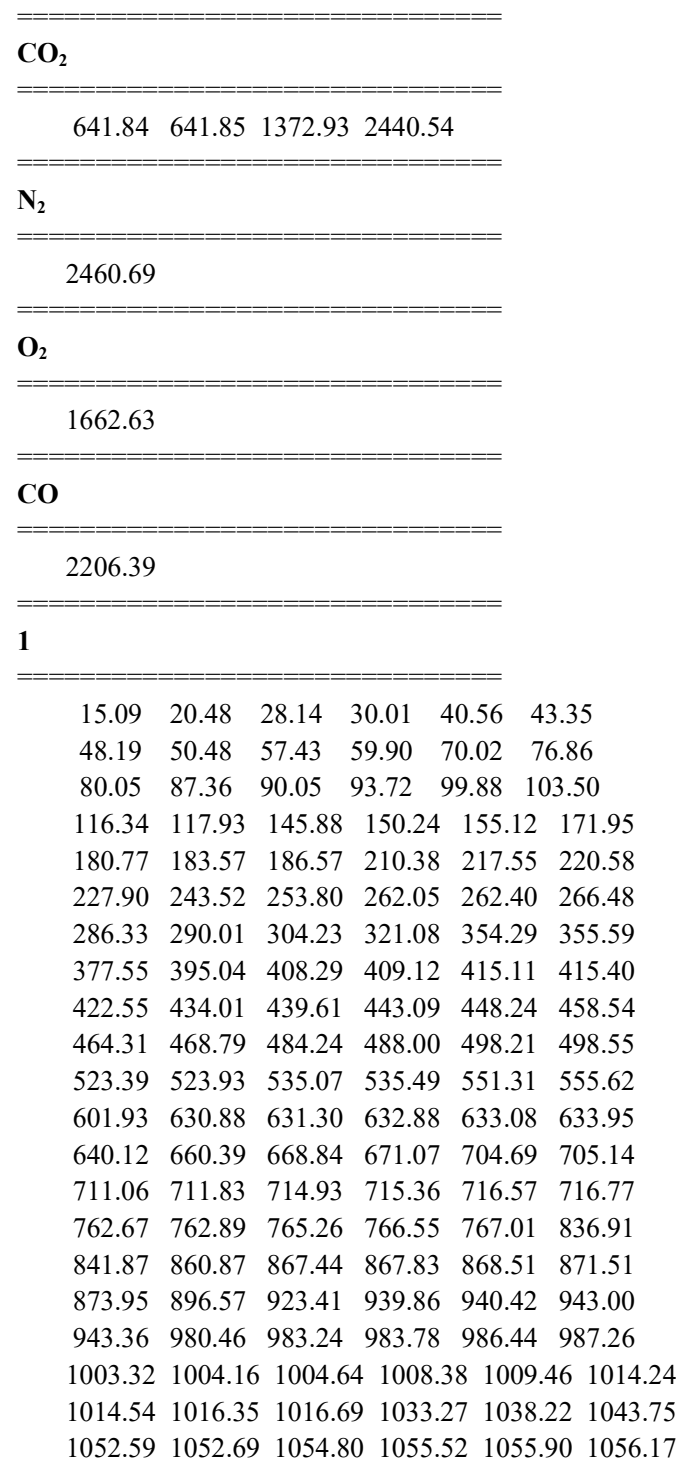

$\begin{array}{llllll}1060.17 & 1088.36 & 1091.67 & 1110.75 & 1111.08 & 1115.97\end{array}$ $\begin{array}{lllllll}1116.55 & 1118.69 & 1119.94 & 1123.87 & 1124.08 & 1193.96\end{array}$ $\begin{array}{lllllll}1196.23 & 1197.14 & 1197.21 & 1198.79 & 1199.57 & 1205.95\end{array}$ $\begin{array}{lllllll}1217.98 & 1219.01 & 1224.83 & 1228.94 & 1231.61 & 1232.09\end{array}$ $\begin{array}{lllllll}1293.03 & 1300.56 & 1322.61 & 1323.50 & 1328.22 & 1328.49\end{array}$ $\begin{array}{lllllll}1332.79 & 1332.92 & 1363.20 & 1363.70 & 1372.15 & 1372.31\end{array}$ $\begin{array}{lllllll}1373.77 & 1378.96 & 1386.51 & 1390.56 & 1420.26 & 1420.31\end{array}$ $\begin{array}{lllllll}1428.99 & 1444.69 & 1473.37 & 1474.08 & 1475.57 & 1476.18\end{array}$ $\begin{array}{lllllll}1478.41 & 1478.95 & 1491.94 & 1495.71 & 1496.93 & 1498.26\end{array}$ $\begin{array}{lllllll}1506.27 & 1518.31 & 1521.22 & 1526.15 & 1526.47 & 1531.94\end{array}$ $\begin{array}{llllllll}1532.14 & 1538.31 & 1547.47 & 1627.57 & 1628.27 & 1629.71\end{array}$ $\begin{array}{lllllll}1630.60 & 1646.80 & 1647.06 & 1648.21 & 1649.24 & 1903.94\end{array}$ $1963.353021 .163023 .66 \quad 3027.773028 .103047 .12$ $\begin{array}{lllllll}3047.71 & 3054.21 & 3054.85 & 3072.85 & 3073.16 & 3097.01\end{array}$ $\begin{array}{lllllll}3098.33 & 3102.68 & 3103.07 & 3108.51 & 3108.79 & 3146.32\end{array}$ $\begin{array}{llllll}3160.11 & 3169.75 & 3169.77 & 3171.74 & 3172.64 & 3177.66\end{array}$ $\begin{array}{lllllll}3177.84 & 3182.51 & 3182.60 & 3188.28 & 3188.29 & 3195.29\end{array}$ $\begin{array}{llllll}3196.30 & 3198.61 & 3198.64 & 3204.97 & 3205.06 & 3205.50\end{array}$ $3206.30 \quad 3211.98 \quad 3212.57$

2

$\begin{array}{llllll}24.66 & 26.37 & 27.66 & 32.94 & 38.37 & 43.79\end{array}$

$\begin{array}{lllllll}50.66 & 52.60 & 56.51 & 62.48 & 65.74 & 68.26\end{array}$

$\begin{array}{lllllll}73.11 & 78.39 & 82.42 & 84.35 & 92.15 & 95.26\end{array}$ $\begin{array}{llllll}108.11 & 110.06 & 137.93 & 143.98 & 145.17 & 160.01\end{array}$ $\begin{array}{lllllll}174.94 & 176.12 & 182.60 & 186.06 & 191.02 & 210.35\end{array}$ $\begin{array}{lllllll}222.73 & 223.23 & 226.33 & 239.90 & 259.89 & 260.48\end{array}$ $\begin{array}{lllllll}261.47 & 266.11 & 285.78 & 294.91 & 304.90 & 331.29\end{array}$ $\begin{array}{llllll}353.45 & 357.35 & 378.91 & 399.52 & 411.42 & 412.17\end{array}$ $\begin{array}{lllllll}414.15 & 414.48 & 428.93 & 434.09 & 436.65 & 442.84\end{array}$ $\begin{array}{lllllll}459.19 & 474.44 & 486.73 & 492.76 & 494.44 & 518.23\end{array}$ $\begin{array}{lllllll}523.95 & 526.31 & 541.83 & 574.51 & 629.23 & 630.82\end{array}$ $\begin{array}{lllllll}630.98 & 631.38 & 635.34 & 652.26 & 663.65 & 667.19\end{array}$ $\begin{array}{lllllll}705.74 & 707.03 & 711.48 & 711.99 & 715.55 & 716.07\end{array}$ $\begin{array}{lllllll}719.42 & 721.12 & 762.27 & 762.50 & 763.78 & 766.00\end{array}$ $\begin{array}{lllllll}766.62 & 830.51 & 832.52 & 864.20 & 866.16 & 866.41\end{array}$ $\begin{array}{lllllll}870.18 & 870.32 & 875.45 & 886.34 & 915.57 & 940.41\end{array}$ $\begin{array}{lllllll}940.73 & 943.94 & 944.49 & 975.47 & 985.10 & 985.78\end{array}$ $\begin{array}{lllllll}986.86 & 988.04 & 1007.36 & 1007.93 & 1008.09 & 1008.18\end{array}$ 
$\begin{array}{llllll}1011.62 & 1013.06 & 1014.29 & 1015.02 & 1015.52 & 1040.83\end{array}$ $\begin{array}{lllllll}1041.17 & 1048.44 & 1050.22 & 1052.67 & 1054.12 & 1054.94\end{array}$ $\begin{array}{lllllll}1056.47 & 1057.97 & 1063.36 & 1081.59 & 1090.65 & 1115.32\end{array}$ $\begin{array}{lllllll}1115.47 & 1115.78 & 1116.25 & 1118.60 & 1120.39 & 1125.39\end{array}$ $\begin{array}{llllllll}1125.53 & 1197.47 & 1198.09 & 1198.54 & 1198.58 & 1198.86\end{array}$ $\begin{array}{llllllll}1209.43 & 1222.18 & 1224.87 & 1227.11 & 1227.50 & 1227.85\end{array}$ $\begin{array}{lllllll}1229.48 & 1231.43 & 1291.03 & 1300.23 & 1325.52 & 1325.93\end{array}$ $\begin{array}{lllllll}1330.43 & 1330.95 & 1334.74 & 1335.05 & 1363.80 & 1369.65\end{array}$ $\begin{array}{lllllll}1369.95 & 1370.27 & 1370.88 & 1375.70 & 1390.08 & 1400.49\end{array}$ $\begin{array}{lllllll}1413.29 & 1418.83 & 1436.30 & 1466.19 & 1469.07 & 1470.14\end{array}$ $\begin{array}{lllllll}1475.74 & 1475.98 & 1477.57 & 1477.62 & 1478.15 & 1491.99\end{array}$ $\begin{array}{lllllll}1494.71 & 1498.21 & 1500.74 & 1508.85 & 1526.41 & 1529.14\end{array}$ $\begin{array}{lllllll}1529.21 & 1530.72 & 1531.01 & 1531.87 & 1556.08 & 1628.03\end{array}$ $\begin{array}{lllllll}1628.53 & 1629.34 & 1629.70 & 1645.55 & 1645.99 & 1647.78\end{array}$ $\begin{array}{lllllll}1647.97 & 3037.71 & 3038.09 & 3038.25 & 3040.15 & 3050.41\end{array}$ $\begin{array}{llllll}3050.82 & 3067.79 & 3068.41 & 3085.38 & 3086.86 & 3098.16\end{array}$ $\begin{array}{lllllll}3099.03 & 3105.18 & 3105.96 & 3138.59 & 3139.07 & 3158.24\end{array}$ $\begin{array}{lllllll}3173.06 & 3173.15 & 3174.35 & 3174.46 & 3175.77 & 3180.17\end{array}$ $\begin{array}{lllllll}3180.30 & 3185.64 & 3185.82 & 3188.84 & 3188.95 & 3194.43\end{array}$ $\begin{array}{llllll}3195.17 & 3199.14 & 3199.16 & 3203.17 & 3203.38 & 3207.31\end{array}$ $3207.893218 .73 \quad 3220.72$

3

$\begin{array}{llllll}22.95 & 28.18 & 28.27 & 35.61 & 43.72 & 48.03\end{array}$ $\begin{array}{lllllll}50.61 & 53.32 & 56.12 & 64.14 & 73.46 & 75.34\end{array}$ $\begin{array}{llllll}80.11 & 84.68 & 90.20 & 93.26 & 100.98 & 102.97\end{array}$ $\begin{array}{llllll}119.16 & 128.74 & 141.51 & 146.05 & 153.64 & 170.33\end{array}$ $\begin{array}{llllll}179.96 & 183.69 & 185.48 & 208.73 & 218.64 & 228.39\end{array}$ $\begin{array}{lllllll}231.72 & 234.27 & 251.00 & 255.76 & 258.38 & 271.76\end{array}$ $\begin{array}{lllllll}278.23 & 300.53 & 316.82 & 355.74 & 360.95 & 363.03\end{array}$ $\begin{array}{llllll}389.74 & 393.66 & 398.08 & 405.41 & 414.28 & 415.07\end{array}$ $\begin{array}{lllllll}415.87 & 416.33 & 425.03 & 432.90 & 446.37 & 456.76\end{array}$ $\begin{array}{lllllll}458.36 & 463.61 & 473.76 & 477.12 & 490.40 & 490.65\end{array}$ $\begin{array}{lllllll}506.12 & 516.23 & 522.43 & 523.91 & 532.81 & 556.65\end{array}$ $\begin{array}{lllllll}586.46 & 626.07 & 628.37 & 628.88 & 631.40 & 633.17\end{array}$ $\begin{array}{lllllll}647.61 & 655.87 & 661.05 & 667.96 & 700.73 & 701.56\end{array}$ $\begin{array}{llllll}711.60 & 712.15 & 713.70 & 714.44 & 714.83 & 715.17\end{array}$ $\begin{array}{llllll}761.54 & 762.19 & 767.02 & 767.35 & 830.81 & 833.90\end{array}$ $\begin{array}{lllllll}837.31 & 854.74 & 867.45 & 867.67 & 869.22 & 869.45\end{array}$ $\begin{array}{llllll}873.35 & 889.38 & 920.12 & 938.25 & 938.63 & 943.14\end{array}$ $\begin{array}{lllllll}944.01 & 973.91 & 982.79 & 983.66 & 987.50 & 988.12\end{array}$ $\begin{array}{lllllll}1002.66 & 1003.20 & 1004.60 & 1005.52 & 1006.02 & 1008.89\end{array}$ $\begin{array}{llllll}1009.38 & 1014.85 & 1015.53 & 1034.71 & 1037.99 & 1043.10\end{array}$ $\begin{array}{lllllll}1047.80 & 1048.89 & 1052.42 & 1053.06 & 1054.12 & 1054.32\end{array}$ $\begin{array}{lllllll}1058.19 & 1090.57 & 1097.51 & 1111.61 & 1112.23 & 1113.78\end{array}$ $\begin{array}{lllllll}1114.94 & 1115.20 & 1115.69 & 1118.86 & 1119.97 & 1194.76\end{array}$ $\begin{array}{lllllll}1196.50 & 1196.61 & 1197.21 & 1198.40 & 1205.28 & 1211.44\end{array}$ $\begin{array}{llllll}1220.23 & 1221.63 & 1223.64 & 1223.82 & 1225.53 & 1226.72\end{array}$ $\begin{array}{llllll}1296.06 & 1304.86 & 1322.18 & 1322.56 & 1328.17 & 1328.99\end{array}$ $\begin{array}{lllllll}1330.14 & 1330.64 & 1361.41 & 1363.26 & 1365.23 & 1366.33\end{array}$ $\begin{array}{lllllll}1368.62 & 1375.56 & 1390.38 & 1394.37 & 1415.83 & 1418.21\end{array}$ $\begin{array}{lllllll}1429.85 & 1462.99 & 1473.96 & 1474.76 & 1475.44 & 1476.66\end{array}$ $\begin{array}{lllllll}1478.13 & 1479.56 & 1488.69 & 1495.14 & 1495.60 & 1498.72\end{array}$ $\begin{array}{lllllll}1503.65 & 1512.31 & 1525.61 & 1526.18 & 1526.94 & 1527.38\end{array}$ $\begin{array}{lllllll}1527.81 & 1538.64 & 1549.22 & 1626.50 & 1627.03 & 1627.58\end{array}$ $\begin{array}{lllllll}1629.05 & 1644.69 & 1645.81 & 1646.16 & 1646.72 & 2096.83\end{array}$ $\begin{array}{lllllll}2136.96 & 3028.28 & 3029.52 & 3030.05 & 3030.39 & 3047.25\end{array}$ $\begin{array}{llllll}3047.60 & 3051.20 & 3052.25 & 3078.16 & 3078.51 & 3095.41\end{array}$ $\begin{array}{llllll}3096.11 & 3096.53 & 3096.77 & 3110.54 & 3111.78 & 3151.30\end{array}$ $\begin{array}{llllll}3167.49 & 3172.73 & 3172.82 & 3172.91 & 3173.02 & 3180.57\end{array}$

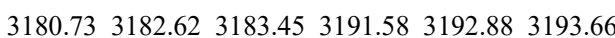
$\begin{array}{llllll}3193.92 & 3199.79 & 3200.00 & 3200.73 & 3200.91 & 3204.97\end{array}$ 3205.213210 .693211 .73

4

$\begin{array}{llllll}13.95 & 18.17 & 18.84 & 20.02 & 23.84 & 24.02\end{array}$ $\begin{array}{llllll}25.56 & 27.65 & 28.05 & 31.09 & 35.44 & 36.35\end{array}$ $\begin{array}{lllllll}40.06 & 43.34 & 45.19 & 46.39 & 49.86 & 51.96\end{array}$ $\begin{array}{lllllll}53.36 & 56.67 & 57.04 & 59.19 & 61.95 & 66.20\end{array}$ $\begin{array}{lllllll}68.99 & 71.82 & 73.48 & 76.67 & 81.58 & 83.20\end{array}$ $\begin{array}{lllllll}88.34 & 92.14 & 94.29 & 101.59 & 105.02 & 108.03\end{array}$ $\begin{array}{lllllll}115.98 & 118.30 & 121.58 & 144.53 & 149.97 & 151.43\end{array}$ $\begin{array}{lllllll}158.47 & 160.10 & 165.01 & 167.51 & 172.28 & 174.24\end{array}$ $\begin{array}{lllllll}176.12 & 178.28 & 183.11 & 187.20 & 189.33 & 192.89\end{array}$ $\begin{array}{lllllll}204.31 & 206.81 & 211.33 & 213.67 & 217.46 & 219.53\end{array}$ $\begin{array}{lllllll}223.51 & 227.07 & 227.47 & 230.60 & 234.59 & 237.55\end{array}$ $\begin{array}{lllllll}245.78 & 253.78 & 255.52 & 256.73 & 258.55 & 264.84\end{array}$ $\begin{array}{lllllll}267.01 & 269.03 & 272.35 & 273.28 & 275.76 & 279.58\end{array}$ $\begin{array}{llllll}284.47 & 287.48 & 309.53 & 316.54 & 334.20 & 345.07\end{array}$ $\begin{array}{lllllll}349.85 & 354.30 & 363.35 & 364.45 & 376.28 & 382.21\end{array}$ $\begin{array}{lllllll}391.45 & 393.53 & 409.03 & 409.33 & 410.61 & 410.81\end{array}$ $\begin{array}{lllllll}412.62 & 413.36 & 414.44 & 414.99 & 416.60 & 417.08\end{array}$ $\begin{array}{lllllll}420.52 & 420.90 & 422.53 & 424.08 & 432.62 & 432.87\end{array}$ $\begin{array}{lllllll}436.96 & 438.10 & 460.62 & 464.18 & 484.33 & 488.31\end{array}$ $\begin{array}{lllllll}491.24 & 494.65 & 497.22 & 502.96 & 504.52 & 515.29\end{array}$ $\begin{array}{lllllll}520.08 & 523.20 & 526.08 & 528.39 & 533.64 & 545.77\end{array}$ $\begin{array}{lllllll}551.10 & 553.98 & 578.24 & 585.13 & 606.21 & 616.15\end{array}$ $\begin{array}{lllllll}629.11 & 630.18 & 630.46 & 630.90 & 631.34 & 631.62\end{array}$ $\begin{array}{lllllll}632.94 & 633.17 & 641.65 & 643.15 & 657.09 & 658.25\end{array}$ $\begin{array}{lllllll}678.92 & 681.10 & 686.11 & 689.32 & 697.57 & 698.32\end{array}$ $\begin{array}{llllll}705.09 & 705.50 & 708.02 & 710.25 & 711.99 & 712.26\end{array}$ $\begin{array}{llllll}713.40 & 714.05 & 714.44 & 715.69 & 716.15 & 720.97\end{array}$ $\begin{array}{llllll}722.72 & 724.38 & 752.64 & 753.12 & 760.01 & 761.60\end{array}$ $\begin{array}{lllllll}764.18 & 764.55 & 765.37 & 765.59 & 766.81 & 767.49\end{array}$ $\begin{array}{lllllll}771.70 & 777.13 & 829.21 & 845.23 & 846.14 & 856.69\end{array}$ $\begin{array}{lllllll}865.82 & 866.20 & 867.38 & 867.97 & 869.81 & 871.18\end{array}$ $\begin{array}{llllll}872.77 & 875.38 & 877.30 & 877.62 & 900.95 & 908.62\end{array}$ $\begin{array}{lllllll}927.25 & 930.85 & 932.18 & 937.33 & 938.24 & 939.50\end{array}$ $\begin{array}{lllllll}939.83 & 943.46 & 948.85 & 949.19 & 964.59 & 967.24\end{array}$ $\begin{array}{lllllll}977.49 & 978.99 & 981.80 & 983.00 & 983.49 & 985.65\end{array}$ $\begin{array}{lllllll}993.33 & 994.32 & 999.85 & 1003.24 & 1003.79 & 1005.42\end{array}$ $\begin{array}{lllllll}1006.27 & 1007.66 & 1008.12 & 1008.69 & 1012.48 & 1012.74\end{array}$ $\begin{array}{lllllll}1013.57 & 1013.68 & 1014.56 & 1014.80 & 1015.16 & 1015.58\end{array}$ $\begin{array}{lllllll}1016.99 & 1020.07 & 1023.56 & 1024.31 & 1032.75 & 1039.33\end{array}$ $\begin{array}{lllllll}1040.35 & 1043.35 & 1047.25 & 1048.67 & 1050.56 & 1051.00\end{array}$ $\begin{array}{lllllll}1051.97 & 1053.28 & 1053.99 & 1054.20 & 1054.75 & 1055.29\end{array}$ $\begin{array}{lllllll}1056.79 & 1057.80 & 1058.71 & 1061.87 & 1068.68 & 1074.48\end{array}$ $\begin{array}{lllllll}1082.67 & 1083.99 & 1093.06 & 1097.87 & 1108.09 & 1108.30\end{array}$ $\begin{array}{lllllll}1111.13 & 1111.32 & 1111.66 & 1111.90 & 1114.08 & 1114.43\end{array}$ $\begin{array}{lllllll}1115.73 & 1116.04 & 1117.84 & 1119.54 & 1120.26 & 1123.36\end{array}$ $\begin{array}{lllllll}1125.00 & 1125.21 & 1194.40 & 1195.88 & 1197.42 & 1197.63\end{array}$ $\begin{array}{lllllll}1197.85 & 1197.88 & 1198.37 & 1198.49 & 1198.80 & 1199.38\end{array}$ $\begin{array}{lllllll}1202.85 & 1212.13 & 1217.11 & 1221.31 & 1221.72 & 1223.04\end{array}$ $\begin{array}{lllllll}1223.74 & 1227.50 & 1228.75 & 1229.01 & 1229.64 & 1230.32\end{array}$ $\begin{array}{lllllll}1232.12 & 1234.57 & 1246.94 & 1250.30 & 1286.96 & 1303.50\end{array}$ $\begin{array}{lllllll}1310.98 & 1311.42 & 1319.08 & 1321.58 & 1323.71 & 1326.84\end{array}$ $\begin{array}{lllllll}1329.70 & 1330.15 & 1330.40 & 1331.30 & 1331.83 & 1333.49\end{array}$ $\begin{array}{lllllll}1339.02 & 1339.82 & 1347.16 & 1356.95 & 1361.28 & 1364.04\end{array}$ $\begin{array}{lllllll}1364.22 & 1365.96 & 1366.20 & 1368.48 & 1373.98 & 1375.96\end{array}$ $\begin{array}{llllll}1378.53 & 1379.01 & 1388.91 & 1391.16 & 1400.88 & 1402.25\end{array}$ 
$\begin{array}{llllll}1418.50 & 1419.64 & 1425.94 & 1430.01 & 1433.48 & 1436.73\end{array}$ $\begin{array}{lllllll}1455.16 & 1460.04 & 1466.73 & 1469.39 & 1472.07 & 1472.50\end{array}$ $\begin{array}{lllllll}1474.98 & 1475.06 & 1476.84 & 1477.39 & 1478.04 & 1478.69\end{array}$ $\begin{array}{lllllll}1478.86 & 1482.36 & 1492.12 & 1493.27 & 1497.00 & 1498.37\end{array}$ $\begin{array}{lllllll}1501.10 & 1502.14 & 1504.11 & 1505.20 & 1510.26 & 1515.90\end{array}$ $\begin{array}{llllllll}1516.41 & 1518.43 & 1519.26 & 1523.74 & 1524.89 & 1525.65\end{array}$ $\begin{array}{lllllll}1526.39 & 1527.41 & 1528.53 & 1529.13 & 1529.64 & 1530.94\end{array}$ $\begin{array}{lllllll}1537.11 & 1538.76 & 1546.63 & 1546.91 & 1622.01 & 1624.59\end{array}$ $\begin{array}{lllllll}1624.84 & 1626.44 & 1626.80 & 1627.66 & 1627.99 & 1628.44\end{array}$ $\begin{array}{lllllll}1643.01 & 1643.26 & 1645.15 & 1645.48 & 1646.19 & 1646.60\end{array}$ $\begin{array}{lllllll}1647.37 & 1647.53 & 3016.93 & 3018.69 & 3020.38 & 3030.15\end{array}$ $\begin{array}{lllllll}3032.61 & 3034.01 & 3035.42 & 3037.83 & 3040.20 & 3047.38\end{array}$ $\begin{array}{lllllll}3058.92 & 3059.36 & 3060.89 & 3063.91 & 3071.83 & 3075.61\end{array}$ $\begin{array}{llllll}3078.46 & 3079.63 & 3080.15 & 3091.96 & 3094.32 & 3094.72\end{array}$ $\begin{array}{llllll}3097.98 & 3098.02 & 3105.05 & 3122.44 & 3126.88 & 3128.33\end{array}$ $\begin{array}{llllll}3135.27 & 3137.99 & 3139.17 & 3143.09 & 3147.00 & 3161.40\end{array}$ $\begin{array}{llllll}3161.66 & 3161.85 & 3163.06 & 3165.91 & 3167.83 & 3168.65\end{array}$ $\begin{array}{lllllll}3170.05 & 3170.26 & 3173.58 & 3173.73 & 3174.97 & 3175.26\end{array}$ $\begin{array}{lllllll}3175.95 & 3177.75 & 3178.80 & 3181.31 & 3181.94 & 3182.42\end{array}$ $\begin{array}{llllll}3183.43 & 3184.76 & 3189.06 & 3189.34 & 3192.16 & 3192.20\end{array}$ $\begin{array}{llllll}3193.33 & 3193.92 & 3193.95 & 3194.58 & 3195.71 & 3201.65\end{array}$ $\begin{array}{llllll}3201.84 & 3202.31 & 3202.78 & 3202.87 & 3203.24 & 3203.64\end{array}$

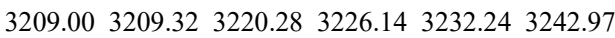

4-cis

$\begin{array}{llllll}12.29 & 13.67 & 16.21 & 16.90 & 18.58 & 20.19\end{array}$

$\begin{array}{llllll}23.13 & 24.55 & 28.03 & 29.23 & 33.68 & 37.21\end{array}$

$\begin{array}{lllllll}39.77 & 42.75 & 45.50 & 47.52 & 49.84 & 51.77\end{array}$

$\begin{array}{llllll}53.07 & 53.55 & 55.60 & 56.82 & 60.02 & 62.60\end{array}$

$\begin{array}{llllll}66.22 & 68.57 & 71.20 & 77.79 & 79.69 & 83.85\end{array}$

$\begin{array}{lllllll}85.75 & 89.12 & 95.58 & 99.07 & 101.35 & 104.90\end{array}$

$\begin{array}{llllll}113.29 & 115.93 & 121.87 & 130.94 & 137.25 & 149.29\end{array}$

$\begin{array}{lllllll}153.07 & 164.41 & 166.38 & 166.68 & 167.67 & 169.02\end{array}$

$\begin{array}{lllllll}176.25 & 180.32 & 182.04 & 185.30 & 186.68 & 191.82\end{array}$ $\begin{array}{llllll}195.11 & 196.94 & 203.63 & 209.97 & 212.59 & 212.80\end{array}$ $\begin{array}{llllll}216.10 & 218.35 & 221.49 & 226.36 & 228.80 & 230.43\end{array}$ $\begin{array}{lllllll}231.79 & 239.11 & 245.84 & 254.80 & 255.86 & 258.68\end{array}$ $\begin{array}{lllllll}259.32 & 260.28 & 266.01 & 273.36 & 277.26 & 285.07\end{array}$ $\begin{array}{lllllll}287.93 & 305.74 & 307.77 & 317.34 & 323.94 & 346.46\end{array}$ $\begin{array}{lllllll}348.26 & 357.58 & 362.21 & 367.78 & 380.72 & 382.94\end{array}$ $\begin{array}{lllllll}384.84 & 386.73 & 403.36 & 404.66 & 407.74 & 408.50\end{array}$ $\begin{array}{lllllll}408.83 & 409.33 & 411.97 & 413.41 & 413.86 & 413.93\end{array}$ $\begin{array}{lllllll}416.13 & 417.83 & 425.90 & 427.49 & 433.78 & 438.96\end{array}$ $\begin{array}{lllllll}440.48 & 441.73 & 459.29 & 463.42 & 486.62 & 487.64\end{array}$ $\begin{array}{lllllll}488.65 & 489.93 & 491.67 & 495.29 & 499.03 & 500.20\end{array}$ $\begin{array}{llllll}522.18 & 522.83 & 525.61 & 526.29 & 534.85 & 536.57\end{array}$ $\begin{array}{lllllll}551.67 & 552.64 & 575.70 & 578.57 & 617.48 & 625.60\end{array}$ $\begin{array}{lllllll}629.55 & 629.85 & 630.24 & 630.82 & 631.45 & 631.85\end{array}$ $\begin{array}{lllllll}632.16 & 632.21 & 640.55 & 643.53 & 658.82 & 660.32\end{array}$ $\begin{array}{llllll}677.32 & 683.14 & 693.20 & 694.34 & 700.31 & 702.06\end{array}$ $\begin{array}{llllll}706.71 & 709.28 & 711.44 & 712.51 & 712.73 & 713.49\end{array}$ $\begin{array}{llllll}714.42 & 715.31 & 715.78 & 715.89 & 725.16 & 725.56\end{array}$ $\begin{array}{llllll}729.47 & 729.90 & 754.05 & 754.82 & 758.63 & 760.37\end{array}$ $\begin{array}{llllll}763.43 & 763.70 & 765.57 & 766.10 & 767.07 & 767.73\end{array}$ $\begin{array}{lllllll}769.99 & 770.84 & 835.01 & 839.91 & 856.04 & 857.52\end{array}$ $\begin{array}{lllllll}863.39 & 868.29 & 868.35 & 869.93 & 870.51 & 870.76\end{array}$ $\begin{array}{llllll}872.49 & 873.87 & 874.22 & 876.74 & 911.48 & 914.39\end{array}$ $\begin{array}{llllll}923.96 & 927.00 & 934.78 & 939.31 & 939.42 & 940.58\end{array}$ $\begin{array}{lllllll}940.83 & 947.03 & 948.66 & 951.19 & 969.39 & 970.61\end{array}$ $\begin{array}{llllll}973.66 & 977.77 & 984.10 & 985.18 & 985.68 & 988.11\end{array}$ $\begin{array}{llllll}990.47 & 991.50 & 998.33 & 1001.33 & 1002.99 & 1004.05\end{array}$ $\begin{array}{lllllll}1005.84 & 1007.04 & 1008.94 & 1009.60 & 1010.62 & 1011.61\end{array}$ $\begin{array}{lllllll}1013.48 & 1013.56 & 1014.23 & 1015.03 & 1015.14 & 1015.48\end{array}$ $\begin{array}{lllllll}1017.97 & 1021.29 & 1023.73 & 1027.09 & 1035.97 & 1037.24\end{array}$ $\begin{array}{lllllll}1042.08 & 1043.08 & 1047.75 & 1048.46 & 1048.99 & 1049.49\end{array}$ $\begin{array}{lllllll}1050.83 & 1052.29 & 1053.42 & 1053.50 & 1053.96 & 1054.10\end{array}$ $\begin{array}{lllllll}1056.71 & 1057.70 & 1058.75 & 1063.28 & 1064.49 & 1066.63\end{array}$ $\begin{array}{lllllll}1081.51 & 1083.16 & 1095.48 & 1097.29 & 1106.19 & 1107.06\end{array}$ $\begin{array}{lllllll}1110.90 & 1111.24 & 1111.28 & 1112.73 & 1113.58 & 1113.83\end{array}$ $\begin{array}{lllllll}1113.97 & 1114.76 & 1115.92 & 1117.53 & 1122.71 & 1124.38\end{array}$ $\begin{array}{lllllll}1126.41 & 1128.15 & 1193.80 & 1194.12 & 1196.25 & 1196.91\end{array}$ $\begin{array}{lllllll}1197.14 & 1197.31 & 1198.04 & 1198.57 & 1199.24 & 1199.95\end{array}$ $\begin{array}{lllllll}1202.66 & 1206.62 & 1216.08 & 1217.93 & 1219.70 & 1220.63\end{array}$ $\begin{array}{lllllll}1223.24 & 1225.67 & 1226.80 & 1226.87 & 1228.47 & 1231.13\end{array}$ $\begin{array}{lllllll}1232.63 & 1233.43 & 1241.15 & 1243.65 & 1295.26 & 1297.42\end{array}$ $\begin{array}{lllllll}1308.08 & 1313.52 & 1316.91 & 1319.71 & 1323.76 & 1324.03\end{array}$ $\begin{array}{lllllll}1325.01 & 1325.53 & 1328.55 & 1331.26 & 1333.09 & 1335.85\end{array}$ $\begin{array}{lllllll}1336.62 & 1337.32 & 1350.38 & 1353.68 & 1359.58 & 1360.33\end{array}$ $\begin{array}{lllllll}1365.85 & 1366.44 & 1367.12 & 1369.73 & 1372.11 & 1372.90\end{array}$ $\begin{array}{lllllll}1373.73 & 1378.44 & 1384.87 & 1398.70 & 1403.29 & 1404.71\end{array}$ $\begin{array}{lllllll}1417.50 & 1419.87 & 1422.97 & 1426.08 & 1429.99 & 1435.54\end{array}$ $\begin{array}{llllllll}1462.33 & 1463.29 & 1466.54 & 1468.51 & 1471.79 & 1472.02\end{array}$ $\begin{array}{llllllll}1474.17 & 1474.65 & 1475.48 & 1475.74 & 1477.13 & 1478.09\end{array}$ $\begin{array}{lllllll}1482.89 & 1485.05 & 1490.84 & 1491.66 & 1496.08 & 1496.29\end{array}$ $\begin{array}{lllllll}1497.07 & 1497.99 & 1502.16 & 1502.99 & 1510.06 & 1512.63\end{array}$ $\begin{array}{lllllll}1513.52 & 1515.46 & 1516.83 & 1517.33 & 1524.12 & 1524.75\end{array}$ $\begin{array}{lllllll}1526.29 & 1527.03 & 1528.18 & 1528.43 & 1529.22 & 1529.47\end{array}$ $\begin{array}{lllllll}1529.95 & 1530.68 & 1544.98 & 1548.68 & 1621.36 & 1622.73\end{array}$ $\begin{array}{lllllll}1623.59 & 1623.65 & 1625.51 & 1625.80 & 1626.32 & 1627.00\end{array}$ $\begin{array}{llllll}1640.26 & 1641.86 & 1644.77 & 1645.19 & 1645.36 & 1646.15\end{array}$ $\begin{array}{lllllll}1646.35 & 1647.25 & 3017.12 & 3020.88 & 3021.50 & 3023.39\end{array}$ $\begin{array}{lllllll}3023.53 & 3026.94 & 3030.35 & 3031.59 & 3044.63 & 3047.25\end{array}$ $\begin{array}{lllllll}3049.37 & 3052.29 & 3053.02 & 3054.87 & 3062.99 & 3064.78\end{array}$ $\begin{array}{lllllll}3075.79 & 3077.44 & 3078.42 & 3080.79 & 3082.99 & 3091.91\end{array}$ $\begin{array}{llllll}3093.03 & 3095.64 & 3106.38 & 3106.62 & 3123.47 & 3123.96\end{array}$ $\begin{array}{lllllll}3126.92 & 3142.86 & 3144.61 & 3147.12 & 3154.28 & 3156.48\end{array}$ $\begin{array}{llllll}3159.17 & 3160.01 & 3162.77 & 3163.16 & 3165.10 & 3168.03\end{array}$ $\begin{array}{lllllll}3169.00 & 3170.42 & 3171.55 & 3171.96 & 3172.43 & 3172.99\end{array}$ $\begin{array}{lllllll}3173.15 & 3178.01 & 3178.56 & 3178.67 & 3179.65 & 3183.02\end{array}$ $\begin{array}{llllll}3183.58 & 3183.84 & 3187.10 & 3187.27 & 3187.78 & 3187.96\end{array}$ $\begin{array}{llllll}3188.00 & 3189.03 & 3190.41 & 3194.47 & 3194.99 & 3195.03\end{array}$ $\begin{array}{lllllll}3195.94 & 3196.01 & 3196.66 & 3198.64 & 3199.75 & 3202.57\end{array}$

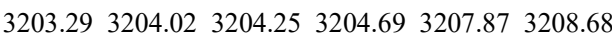

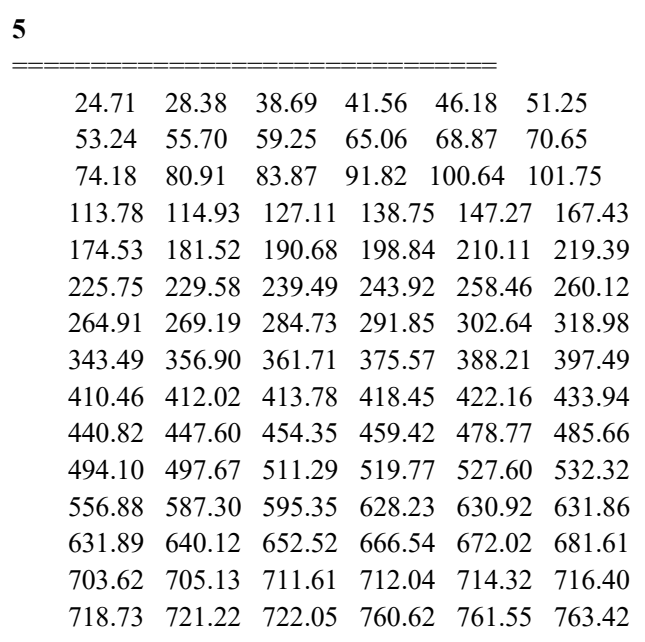


$\begin{array}{llllll}768.10 & 768.76 & 807.67 & 814.62 & 844.30 & 859.77\end{array}$ $\begin{array}{lllllll}862.11 & 864.32 & 872.75 & 874.66 & 874.79 & 886.34\end{array}$ $\begin{array}{lllllll}910.80 & 929.57 & 937.76 & 946.69 & 953.10 & 974.31\end{array}$ $\begin{array}{llllll}977.18 & 980.87 & 991.49 & 995.56 & 1002.65 & 1003.07\end{array}$ $\begin{array}{lllllll}1003.79 & 1010.13 & 1013.07 & 1014.55 & 1015.69 & 1020.59\end{array}$ $\begin{array}{lllllll}1024.05 & 1025.02 & 1035.86 & 1044.05 & 1048.87 & 1052.64\end{array}$ $\begin{array}{lllllll}1053.39 & 1053.70 & 1055.65 & 1057.06 & 1057.59 & 1062.54\end{array}$ $\begin{array}{lllllll}1084.55 & 1094.40 & 1112.30 & 1115.44 & 1116.73 & 1117.34\end{array}$ $\begin{array}{lllllll}1120.72 & 1121.87 & 1122.53 & 1126.66 & 1195.83 & 1197.99\end{array}$ $\begin{array}{lllllll}1198.25 & 1199.43 & 1200.28 & 1206.90 & 1209.40 & 1223.65\end{array}$ $\begin{array}{lllllll}1223.89 & 1225.28 & 1228.29 & 1229.87 & 1232.50 & 1234.42\end{array}$ $\begin{array}{lllllll}1295.21 & 1301.72 & 1323.47 & 1326.17 & 1331.26 & 1332.86\end{array}$ $\begin{array}{lllllll}1334.43 & 1338.55 & 1362.99 & 1365.35 & 1367.09 & 1374.07\end{array}$ $\begin{array}{lllllll}1376.03 & 1377.26 & 1389.02 & 1395.88 & 1413.72 & 1418.94\end{array}$ $\begin{array}{lllllll}1427.60 & 1441.71 & 1449.96 & 1473.92 & 1475.39 & 1477.09\end{array}$ $\begin{array}{lllllll}1479.75 & 1480.00 & 1488.06 & 1491.53 & 1499.26 & 1501.49\end{array}$ $\begin{array}{lllllll}1505.98 & 1516.06 & 1523.92 & 1527.80 & 1528.24 & 1529.63\end{array}$ $\begin{array}{llllllll}1531.22 & 1542.74 & 1547.70 & 1625.16 & 1627.51 & 1628.61\end{array}$ $\begin{array}{llllllll}1629.32 & 1645.78 & 1646.47 & 1647.42 & 1647.73 & 1802.56\end{array}$ $\begin{array}{lllllll}1925.52 & 3039.94 & 3041.09 & 3043.91 & 3046.67 & 3056.55\end{array}$ $\begin{array}{llllll}3058.15 & 3063.29 & 3067.70 & 3085.53 & 3088.16 & 3096.81\end{array}$ $\begin{array}{llllll}3106.90 & 3125.84 & 3129.39 & 3139.98 & 3159.71 & 3171.70\end{array}$ $\begin{array}{lllllll}3172.50 & 3175.04 & 3175.59 & 3176.49 & 3176.66 & 3181.99\end{array}$ $\begin{array}{llllll}3182.25 & 3183.57 & 3187.18 & 3192.40 & 3193.18 & 3193.54\end{array}$ $\begin{array}{llllll}3196.81 & 3199.37 & 3203.30 & 3203.40 & 3203.76 & 3204.49\end{array}$ $3208.103227 .66 \quad 3236.09$

\section{F}

$\begin{array}{llllll}26.47 & 31.03 & 34.90 & 42.84 & 44.58 & 46.22\end{array}$

$\begin{array}{llllll}51.37 & 54.28 & 61.96 & 70.60 & 70.83 & 77.09\end{array}$

$\begin{array}{lllllll}84.51 & 89.81 & 95.05 & 110.72 & 114.62 & 121.41\end{array}$

$\begin{array}{lllllll}130.84 & 142.91 & 153.45 & 168.05 & 174.32 & 177.20\end{array}$

$\begin{array}{lllllll}179.33 & 192.39 & 194.43 & 197.63 & 214.87 & 222.62\end{array}$

$\begin{array}{lllllll}224.52 & 226.86 & 232.02 & 244.80 & 257.45 & 258.26\end{array}$

$\begin{array}{lllllll}260.14 & 265.73 & 281.77 & 293.86 & 304.36 & 319.88\end{array}$

$\begin{array}{lllllll}331.77 & 357.38 & 366.66 & 368.46 & 380.42 & 400.74\end{array}$

$\begin{array}{lllllll}407.19 & 412.08 & 414.20 & 414.36 & 418.06 & 431.80\end{array}$

$\begin{array}{lllllll}439.59 & 441.56 & 447.98 & 457.19 & 469.36 & 483.86\end{array}$

$\begin{array}{lllllll}491.99 & 494.69 & 503.18 & 520.36 & 524.85 & 527.58\end{array}$ $\begin{array}{lllllll}550.20 & 555.89 & 593.70 & 612.87 & 627.46 & 628.42\end{array}$ $\begin{array}{lllllll}631.00 & 632.48 & 641.96 & 656.51 & 670.54 & 672.37\end{array}$ $\begin{array}{llllll}702.46 & 704.68 & 707.50 & 710.96 & 713.10 & 715.71\end{array}$ $\begin{array}{llllll}718.35 & 719.11 & 724.39 & 736.63 & 763.00 & 763.81\end{array}$ $\begin{array}{lllllll}766.64 & 767.54 & 768.96 & 826.00 & 844.52 & 864.21\end{array}$ $\begin{array}{lllllll}865.29 & 867.94 & 871.38 & 875.37 & 877.07 & 893.23\end{array}$ $\begin{array}{lllllll}918.37 & 941.06 & 943.97 & 946.69 & 947.69 & 979.28\end{array}$ $\begin{array}{lllllll}983.48 & 988.13 & 990.91 & 993.76 & 1002.50 & 1006.54\end{array}$ $\begin{array}{lllllll}1008.76 & 1010.95 & 1013.08 & 1014.64 & 1015.00 & 1017.64\end{array}$ $\begin{array}{lllllll}1024.63 & 1038.70 & 1043.01 & 1049.37 & 1049.54 & 1050.65\end{array}$ $\begin{array}{lllllll}1054.94 & 1056.37 & 1057.23 & 1058.46 & 1067.24 & 1080.82\end{array}$ $\begin{array}{lllllll}1092.91 & 1114.74 & 1115.91 & 1117.10 & 1118.99 & 1120.01\end{array}$ $\begin{array}{lllllll}1122.15 & 1122.18 & 1130.79 & 1172.67 & 1196.87 & 1198.19\end{array}$ $\begin{array}{lllllll}1198.56 & 1199.00 & 1199.41 & 1211.11 & 1216.69 & 1222.26\end{array}$ $\begin{array}{lllllll}1225.93 & 1228.44 & 1229.06 & 1230.51 & 1231.96 & 1233.69\end{array}$ $\begin{array}{lllllll}1297.77 & 1313.54 & 1325.61 & 1326.09 & 1330.95 & 1331.31\end{array}$ $\begin{array}{lllllll}1333.78 & 1336.36 & 1368.32 & 1372.21 & 1372.93 & 1373.94\end{array}$ $\begin{array}{llllllll}1374.82 & 1384.10 & 1393.05 & 1407.60 & 1418.58 & 1432.99\end{array}$ $\begin{array}{lllllll}1435.65 & 1462.72 & 1466.63 & 1474.95 & 1475.84 & 1477.11\end{array}$ $\begin{array}{lllllll}1478.57 & 1479.67 & 1489.85 & 1494.33 & 1499.63 & 1501.66\end{array}$ $\begin{array}{llllll}1506.78 & 1516.52 & 1529.06 & 1529.53 & 1531.06 & 1532.16\end{array}$ $\begin{array}{llllll}1533.13 & 1542.29 & 1561.22 & 1627.74 & 1628.74 & 1629.46\end{array}$ $\begin{array}{llllllll}1630.09 & 1646.51 & 1647.04 & 1648.17 & 1648.70 & 1814.90\end{array}$ $\begin{array}{lllllll}1874.76 & 3035.45 & 3035.90 & 3040.40 & 3043.55 & 3053.54\end{array}$ $\begin{array}{lllllll}3055.60 & 3077.40 & 3081.44 & 3083.83 & 3088.53 & 3091.49\end{array}$ $\begin{array}{lllllll}3107.71 & 3113.94 & 3114.19 & 3156.98 & 3159.26 & 3173.39\end{array}$ $\begin{array}{lllllll}3173.50 & 3175.80 & 3176.24 & 3177.63 & 3179.11 & 3183.59\end{array}$ $\begin{array}{lllllll}3184.07 & 3185.06 & 3186.68 & 3193.17 & 3194.21 & 3196.00\end{array}$ $\begin{array}{llllll}3197.41 & 3201.41 & 3201.99 & 3204.08 & 3208.61 & 3209.26\end{array}$ $3212.10 \quad 3216.70 \quad 3242.25$

\section{F-TS}

$\begin{array}{llllll}-162.23 & 13.11 & 15.23 & 22.47 & 29.31 & 32.93\end{array}$

$\begin{array}{llllll}37.36 & 39.75 & 45.78 & 49.28 & 53.38 & 62.12\end{array}$

$\begin{array}{lllllll}65.27 & 78.22 & 84.02 & 90.28 & 99.45 & 109.21\end{array}$

$\begin{array}{llllll}122.17 & 129.47 & 146.54 & 149.57 & 155.93 & 158.83\end{array}$

$\begin{array}{lllllll}162.20 & 172.11 & 182.96 & 185.68 & 189.58 & 201.94\end{array}$

$\begin{array}{lllllll}210.06 & 214.36 & 217.94 & 229.68 & 250.54 & 253.54\end{array}$ $\begin{array}{lllllll}257.32 & 263.83 & 274.01 & 281.06 & 296.01 & 302.86\end{array}$ $\begin{array}{lllllll}306.23 & 360.01 & 361.90 & 366.50 & 369.51 & 379.16\end{array}$ $\begin{array}{lllllll}393.02 & 401.92 & 407.83 & 412.45 & 412.89 & 419.72\end{array}$ $\begin{array}{lllllll}420.19 & 430.04 & 444.86 & 455.60 & 466.15 & 471.67\end{array}$ $\begin{array}{lllllll}484.70 & 490.46 & 495.55 & 512.44 & 522.95 & 530.84\end{array}$ $\begin{array}{llllll}535.40 & 548.52 & 578.29 & 625.67 & 630.27 & 631.09\end{array}$ $\begin{array}{lllllll}631.34 & 632.58 & 639.61 & 647.36 & 664.88 & 671.80\end{array}$ $\begin{array}{lllllll}675.34 & 704.84 & 706.59 & 709.91 & 710.05 & 712.02\end{array}$ $\begin{array}{llllll}714.32 & 715.17 & 717.02 & 755.99 & 759.29 & 765.51\end{array}$ $\begin{array}{lllllll}765.87 & 766.11 & 771.37 & 833.66 & 838.92 & 856.74\end{array}$ $\begin{array}{lllllll}861.00 & 863.18 & 866.36 & 868.17 & 879.01 & 889.93\end{array}$ $\begin{array}{llllll}919.74 & 925.06 & 932.65 & 938.38 & 941.46 & 970.98\end{array}$ $\begin{array}{llllll}975.74 & 976.87 & 982.16 & 985.25 & 999.39 & 1001.68\end{array}$ $\begin{array}{lllllll}1004.33 & 1005.70 & 1007.90 & 1014.57 & 1014.82 & 1015.22\end{array}$ $\begin{array}{lllllll}1016.93 & 1035.96 & 1037.64 & 1044.44 & 1050.60 & 1052.48\end{array}$ $\begin{array}{lllllll}1054.60 & 1054.98 & 1055.61 & 1056.01 & 1061.75 & 1089.11\end{array}$ $\begin{array}{lllllll}1092.76 & 1110.78 & 1112.08 & 1114.60 & 1116.04 & 1117.53\end{array}$ $\begin{array}{lllllll}1118.88 & 1122.85 & 1125.35 & 1196.91 & 1197.21 & 1197.95\end{array}$ $\begin{array}{lllllll}1198.16 & 1199.59 & 1201.47 & 1209.92 & 1216.35 & 1219.84\end{array}$ $\begin{array}{lllllll}1222.48 & 1226.61 & 1226.99 & 1228.96 & 1234.10 & 1295.05\end{array}$ $\begin{array}{lllllll}1305.52 & 1312.47 & 1321.42 & 1322.24 & 1327.78 & 1329.67\end{array}$ $\begin{array}{lllllll}1330.95 & 1332.59 & 1361.99 & 1362.76 & 1367.21 & 1368.69\end{array}$ $\begin{array}{lllllll}1374.07 & 1378.23 & 1384.20 & 1392.68 & 1413.52 & 1420.76\end{array}$ $\begin{array}{lllllll}1434.23 & 1461.28 & 1463.90 & 1474.21 & 1474.94 & 1476.82\end{array}$ $\begin{array}{lllllll}1477.51 & 1478.00 & 1478.53 & 1495.11 & 1497.53 & 1498.83\end{array}$ $\begin{array}{lllllll}1505.76 & 1506.90 & 1520.64 & 1526.32 & 1527.00 & 1528.58\end{array}$ $\begin{array}{lllllll}1529.84 & 1531.67 & 1546.74 & 1628.40 & 1628.58 & 1630.15\end{array}$ $\begin{array}{lllllll}1630.26 & 1646.78 & 1647.14 & 1648.99 & 1649.24 & 1722.58\end{array}$ $\begin{array}{lllllll}2132.45 & 3022.01 & 3023.77 & 3030.88 & 3031.86 & 3051.70\end{array}$ $\begin{array}{llllll}3053.95 & 3055.21 & 3058.02 & 3076.18 & 3076.72 & 3085.39\end{array}$ $\begin{array}{llllll}3087.39 & 3106.81 & 3110.02 & 3111.42 & 3114.69 & 3146.69\end{array}$ $\begin{array}{lllllll}3161.15 & 3172.05 & 3173.38 & 3175.07 & 3175.14 & 3179.61\end{array}$ $\begin{array}{lllllll}3181.76 & 3182.23 & 3186.72 & 3190.25 & 3191.63 & 3194.45\end{array}$ $\begin{array}{llllll}3196.96 & 3201.30 & 3201.85 & 3203.24 & 3203.75 & 3208.89\end{array}$ $3211.93 \quad 3223.98 \quad 3224.49$

\begin{tabular}{|c|c|c|c|c|c|}
\hline G & & & & & \\
\hline 18.99 & 26.79 & 30.69 & 32.20 & 36.56 & 40.21 \\
\hline 45.35 & 54.17 & 56.11 & 63.95 & 68.43 & 73.61 \\
\hline 76.67 & 90.85 & 95.72 & 101.11 & 116.01 & 120.91 \\
\hline 133.88 & 148.30 & 152.93 & $\begin{array}{ll}3 & 161.56\end{array}$ & $6 \quad 171.55$ & $5 \quad 185.50$ \\
\hline 190.18 & 194.84 & 195.22 & $2 \quad 206.01$ & $\begin{array}{ll}1 & 210.29\end{array}$ & $\begin{array}{ll}9 & 219.60\end{array}$ \\
\hline
\end{tabular}


$\begin{array}{lllllll}225.97 & 226.67 & 237.58 & 249.44 & 256.62 & 263.57\end{array}$ $\begin{array}{lllllll}267.59 & 283.37 & 301.28 & 311.10 & 329.29 & 340.98\end{array}$ $\begin{array}{lllllll}352.79 & 359.61 & 376.33 & 399.70 & 405.86 & 409.61\end{array}$ $\begin{array}{lllllll}409.75 & 411.55 & 418.59 & 419.39 & 421.86 & 426.02\end{array}$ $\begin{array}{llllll}443.43 & 468.40 & 480.29 & 488.67 & 492.91 & 502.17\end{array}$ $\begin{array}{lllllll}509.53 & 513.10 & 524.41 & 530.14 & 546.15 & 551.02\end{array}$ $\begin{array}{llllll}587.46 & 630.26 & 631.15 & 631.71 & 631.91 & 641.91\end{array}$ $\begin{array}{lllllll}656.68 & 658.95 & 670.50 & 671.92 & 706.63 & 708.43\end{array}$ $\begin{array}{lllllll}708.88 & 712.71 & 712.93 & 716.56 & 717.73 & 720.82\end{array}$ $\begin{array}{llllll}758.09 & 759.56 & 761.45 & 765.11 & 765.60 & 773.26\end{array}$ $\begin{array}{lllllll}775.37 & 783.78 & 833.93 & 843.69 & 857.56 & 865.12\end{array}$ $\begin{array}{lllllll}867.82 & 868.65 & 871.93 & 876.99 & 877.43 & 894.74\end{array}$ $\begin{array}{llllll}921.74 & 929.49 & 941.85 & 942.72 & 946.32 & 974.30\end{array}$ $\begin{array}{lllllll}981.45 & 984.01 & 987.97 & 989.36 & 1001.83 & 1004.27\end{array}$ $\begin{array}{lllllll}1007.46 & 1007.98 & 1014.57 & 1015.27 & 1015.46 & 1016.65\end{array}$ $\begin{array}{lllllll}1029.22 & 1044.07 & 1047.12 & 1052.31 & 1053.69 & 1054.07\end{array}$ $\begin{array}{lllllll}1055.19 & 1056.96 & 1064.52 & 1069.08 & 1088.26 & 1100.70\end{array}$ $\begin{array}{lllllll}1112.28 & 1113.21 & 1117.37 & 1119.13 & 1119.96 & 1120.50\end{array}$ $\begin{array}{lllllll}1123.72 & 1125.64 & 1180.85 & 1197.12 & 1197.36 & 1199.54\end{array}$ $\begin{array}{lllllll}1199.93 & 1201.40 & 1205.46 & 1210.04 & 1221.79 & 1223.13\end{array}$ $\begin{array}{lllllll}1224.72 & 1227.15 & 1230.86 & 1235.11 & 1246.76 & 1299.66\end{array}$ $\begin{array}{lllllll}1312.85 & 1319.71 & 1325.08 & 1325.16 & 1328.29 & 1329.45\end{array}$ $\begin{array}{lllllll}1333.49 & 1333.73 & 1364.12 & 1364.73 & 1368.95 & 1370.23\end{array}$ $\begin{array}{lllllll}1375.00 & 1381.11 & 1388.05 & 1397.42 & 1414.43 & 1421.04\end{array}$ $\begin{array}{lllllll}1464.45 & 1469.67 & 1470.54 & 1475.33 & 1476.27 & 1477.92\end{array}$ $\begin{array}{lllllll}1478.48 & 1478.50 & 1496.55 & 1498.51 & 1501.28 & 1508.49\end{array}$ $\begin{array}{lllllll}1513.95 & 1516.40 & 1527.26 & 1528.71 & 1530.81 & 1532.31\end{array}$ $\begin{array}{lllllll}1533.84 & 1536.73 & 1563.83 & 1626.73 & 1628.87 & 1630.33\end{array}$ $\begin{array}{llllllll}1631.38 & 1645.64 & 1645.91 & 1649.81 & 1650.30 & 1691.21\end{array}$ $\begin{array}{lllllll}1882.47 & 2186.44 & 2251.87 & 2399.70 & 3027.70 & 3031.67\end{array}$ $\begin{array}{lllllll}3033.41 & 3042.23 & 3045.91 & 3051.23 & 3061.59 & 3073.95\end{array}$ $\begin{array}{llllll}3075.67 & 3091.76 & 3092.78 & 3106.62 & 3110.09 & 3114.93\end{array}$ $\begin{array}{lllllll}3151.64 & 3172.62 & 3174.07 & 3177.44 & 3177.47 & 3181.24\end{array}$ $\begin{array}{llllll}3182.26 & 3187.39 & 3188.62 & 3192.45 & 3195.03 & 3196.41\end{array}$ $\begin{array}{llllll}3199.43 & 3201.90 & 3202.65 & 3205.81 & 3207.27 & 3209.18\end{array}$ 3212.193214 .793228 .25

\section{G-TS}

$\begin{array}{llllll}-424.65 & 15.61 & 27.71 & 29.20 & 39.54 & 42.05\end{array}$ $\begin{array}{llllll}46.34 & 53.50 & 54.20 & 55.24 & 64.88 & 67.03\end{array}$ $\begin{array}{lllllll}71.52 & 79.80 & 85.16 & 89.68 & 90.87 & 105.05\end{array}$ $\begin{array}{llllll}118.32 & 124.74 & 128.40 & 136.93 & 142.67 & 167.38\end{array}$ $\begin{array}{lllllll}172.77 & 179.26 & 183.87 & 185.92 & 193.43 & 210.87\end{array}$ $\begin{array}{lllllll}220.60 & 222.22 & 227.15 & 234.61 & 239.44 & 249.87\end{array}$ $\begin{array}{llllll}251.76 & 259.83 & 262.86 & 281.66 & 285.10 & 302.14\end{array}$ $\begin{array}{llllll}306.32 & 332.73 & 351.78 & 363.40 & 376.47 & 383.72\end{array}$ $\begin{array}{lllllll}389.25 & 412.34 & 412.46 & 414.04 & 415.84 & 416.34\end{array}$ $\begin{array}{lllllll}428.18 & 434.76 & 441.03 & 449.16 & 461.25 & 477.61\end{array}$ $\begin{array}{lllllll}489.17 & 493.92 & 498.83 & 518.72 & 525.33 & 527.91\end{array}$ $\begin{array}{lllllll}537.68 & 548.11 & 556.33 & 585.41 & 628.71 & 630.18\end{array}$ $\begin{array}{lllllll}631.18 & 631.94 & 642.84 & 655.32 & 667.65 & 671.22\end{array}$ $\begin{array}{lllllll}684.17 & 687.31 & 704.69 & 707.27 & 713.73 & 714.55\end{array}$ $\begin{array}{llllll}715.15 & 716.11 & 718.65 & 720.16 & 761.17 & 763.53\end{array}$ $\begin{array}{llllll}765.06 & 768.11 & 774.17 & 780.37 & 831.43 & 834.14\end{array}$ $\begin{array}{lllllll}862.74 & 867.86 & 870.99 & 871.46 & 874.90 & 876.47\end{array}$ $\begin{array}{lllllll}889.80 & 919.22 & 938.34 & 940.99 & 947.98 & 948.48\end{array}$ $\begin{array}{lllllll}979.16 & 984.15 & 988.20 & 988.53 & 993.09 & 1003.19\end{array}$ $\begin{array}{llllll}1007.38 & 1010.39 & 1011.01 & 1013.35 & 1015.94 & 1016.57\end{array}$ $\begin{array}{lllllll}1017.56 & 1019.09 & 1040.39 & 1042.45 & 1047.52 & 1050.68\end{array}$ $\begin{array}{lllllll}1052.57 & 1053.70 & 1054.62 & 1055.11 & 1058.13 & 1062.74\end{array}$ $\begin{array}{llllll}1092.01 & 1095.18 & 1113.93 & 1115.44 & 1115.84 & 1117.78\end{array}$ $\begin{array}{lllllll}1117.99 & 1119.64 & 1123.42 & 1124.84 & 1197.82 & 1198.39\end{array}$ $\begin{array}{lllllll}1198.68 & 1199.36 & 1202.06 & 1205.81 & 1217.89 & 1222.79\end{array}$ $\begin{array}{lllllll}1225.00 & 1228.84 & 1229.78 & 1230.83 & 1233.99 & 1269.04\end{array}$ $\begin{array}{lllllll}1293.50 & 1303.44 & 1324.31 & 1325.50 & 1328.91 & 1329.35\end{array}$ $\begin{array}{lllllll}1332.36 & 1333.74 & 1364.29 & 1370.12 & 1370.80 & 1373.30\end{array}$ $\begin{array}{lllllll}1374.00 & 1379.70 & 1390.75 & 1400.13 & 1418.13 & 1420.42\end{array}$ $\begin{array}{lllllll}1434.88 & 1469.41 & 1470.92 & 1475.70 & 1475.82 & 1476.75\end{array}$ $\begin{array}{llllllll}1477.11 & 1477.72 & 1490.22 & 1495.43 & 1496.94 & 1500.41\end{array}$ $\begin{array}{llllllll}1501.98 & 1508.67 & 1528.69 & 1529.18 & 1530.49 & 1530.72\end{array}$ $\begin{array}{lllllll}1532.40 & 1541.68 & 1555.39 & 1627.98 & 1628.93 & 1629.56\end{array}$ $\begin{array}{llllllll}1629.72 & 1646.34 & 1647.07 & 1647.95 & 1648.40 & 1800.08\end{array}$ $\begin{array}{lllllll}2092.28 & 3031.15 & 3033.00 & 3034.63 & 3035.25 & 3045.20\end{array}$ $\begin{array}{lllllll}3045.66 & 3050.13 & 3072.50 & 3079.81 & 3085.05 & 3088.46\end{array}$ $\begin{array}{lllllll}3092.04 & 3093.03 & 3096.70 & 3108.22 & 3148.22 & 3163.57\end{array}$ $\begin{array}{lllllll}3165.98 & 3170.82 & 3172.48 & 3173.94 & 3174.27 & 3181.99\end{array}$ $\begin{array}{lllllll}3182.09 & 3182.58 & 3186.13 & 3192.20 & 3193.73 & 3194.54\end{array}$ 3196.223200 .843202 .613202 .763203 .713205 .92 3206.943207 .613245 .08

$\mathbf{H}$

$\begin{array}{llllll}18.39 & 22.00 & 26.89 & 34.38 & 41.34 & 46.49\end{array}$ $\begin{array}{llllll}53.92 & 55.24 & 56.20 & 61.71 & 67.72 & 76.72\end{array}$ $\begin{array}{lllllll}79.09 & 84.42 & 96.59 & 98.38 & 104.06 & 107.75\end{array}$ $\begin{array}{llllll}119.84 & 133.58 & 140.06 & 148.32 & 157.90 & 170.36\end{array}$ $\begin{array}{lllllll}173.93 & 184.05 & 194.26 & 201.58 & 204.38 & 208.43\end{array}$ $\begin{array}{lllllll}215.68 & 228.97 & 229.14 & 237.69 & 251.23 & 259.08\end{array}$ $\begin{array}{llllll}261.39 & 271.30 & 273.46 & 282.58 & 294.95 & 303.38\end{array}$ $\begin{array}{lllllll}316.85 & 329.57 & 338.02 & 341.35 & 368.79 & 373.25\end{array}$ $\begin{array}{lllllll}377.64 & 386.57 & 403.70 & 404.90 & 411.30 & 411.95\end{array}$ $\begin{array}{lllllll}417.99 & 426.42 & 430.57 & 438.38 & 439.69 & 469.90\end{array}$ $\begin{array}{llllll}475.09 & 485.84 & 491.02 & 495.94 & 512.53 & 524.70\end{array}$ $\begin{array}{lllllll}528.65 & 532.72 & 551.45 & 590.28 & 629.09 & 629.54\end{array}$ $\begin{array}{lllllll}629.95 & 632.14 & 639.22 & 653.83 & 659.02 & 665.99\end{array}$ $\begin{array}{lllllll}672.03 & 685.40 & 699.66 & 707.85 & 708.90 & 712.41\end{array}$ $\begin{array}{llllll}718.93 & 721.16 & 724.96 & 727.22 & 736.59 & 748.18\end{array}$ $\begin{array}{llllll}757.19 & 760.98 & 762.41 & 763.62 & 766.10 & 801.78\end{array}$ $\begin{array}{lllllll}813.87 & 825.27 & 840.57 & 852.58 & 859.69 & 863.62\end{array}$ $\begin{array}{lllllll}868.04 & 872.55 & 874.88 & 885.67 & 913.10 & 929.18\end{array}$ $\begin{array}{lllllll}938.38 & 946.09 & 947.21 & 972.14 & 979.23 & 980.67\end{array}$ $\begin{array}{lllllll}987.76 & 994.96 & 999.14 & 1001.97 & 1008.55 & 1009.27\end{array}$ $\begin{array}{lllllll}1012.41 & 1015.68 & 1016.35 & 1016.82 & 1017.88 & 1021.64\end{array}$ $\begin{array}{lllllll}1024.57 & 1035.19 & 1040.44 & 1047.80 & 1051.70 & 1054.36\end{array}$ $\begin{array}{lllllll}1055.10 & 1055.68 & 1056.45 & 1059.41 & 1060.39 & 1077.69\end{array}$ $\begin{array}{lllllll}1088.33 & 1115.04 & 1115.95 & 1118.60 & 1121.26 & 1125.43\end{array}$ $\begin{array}{lllllll}1127.53 & 1128.03 & 1128.37 & 1191.92 & 1196.95 & 1198.21\end{array}$ $\begin{array}{lllllll}1199.15 & 1199.66 & 1200.83 & 1201.42 & 1216.03 & 1221.94\end{array}$ $\begin{array}{lllllll}1225.29 & 1226.26 & 1231.10 & 1232.94 & 1233.72 & 1234.19\end{array}$ $\begin{array}{lllllll}1303.76 & 1315.51 & 1326.39 & 1326.93 & 1331.82 & 1333.24\end{array}$ $\begin{array}{lllllll}1337.65 & 1337.69 & 1366.20 & 1372.52 & 1375.28 & 1377.01\end{array}$ $\begin{array}{lllllll}1377.70 & 1383.40 & 1397.89 & 1403.91 & 1421.13 & 1429.93\end{array}$ $\begin{array}{lllllll}1439.17 & 1457.76 & 1463.91 & 1468.99 & 1475.90 & 1476.58\end{array}$ $\begin{array}{lllllll}1479.74 & 1480.89 & 1490.94 & 1496.07 & 1499.83 & 1501.64\end{array}$ $\begin{array}{llllllll}1510.71 & 1516.43 & 1524.04 & 1529.22 & 1530.68 & 1531.59\end{array}$ $\begin{array}{lllllll}1531.63 & 1545.58 & 1549.85 & 1627.59 & 1629.61 & 1630.02\end{array}$ $\begin{array}{lllllll}1632.50 & 1648.47 & 1648.52 & 1649.18 & 1649.21 & 1815.73\end{array}$ $\begin{array}{lllllll}1839.23 & 3036.37 & 3038.94 & 3043.60 & 3044.46 & 3061.35\end{array}$ $\begin{array}{lllllll}3062.22 & 3077.47 & 3087.31 & 3090.86 & 3108.88 & 3120.78\end{array}$ $\begin{array}{lllllll}3123.59 & 3124.23 & 3137.64 & 3153.23 & 3172.64 & 3173.58\end{array}$ $\begin{array}{llllll}3174.40 & 3176.66 & 3177.12 & 3177.46 & 3181.87 & 3186.85\end{array}$ 


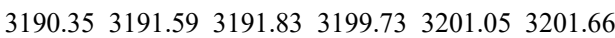
$\begin{array}{llllll}3202.93 & 3207.48 & 3207.54 & 3214.34 & 3215.45 & 3217.51\end{array}$ $3221.64 \quad 3224.10 \quad 3242.82$

I

$\begin{array}{llllll}10.87 & 29.74 & 35.09 & 36.19 & 40.69 & 44.87\end{array}$

$\begin{array}{lllllll}51.47 & 55.40 & 56.46 & 58.97 & 66.13 & 70.33\end{array}$

$\begin{array}{lllllll}71.22 & 85.04 & 86.68 & 92.07 & 94.09 & 101.95\end{array}$

$\begin{array}{llllll}108.64 & 111.41 & 119.43 & 124.83 & 140.19 & 158.41\end{array}$

$\begin{array}{llllll}160.05 & 168.14 & 175.73 & 183.17 & 190.34 & 199.53\end{array}$

$\begin{array}{llllll}201.75 & 212.82 & 217.50 & 219.85 & 228.51 & 234.07\end{array}$

$\begin{array}{llllll}250.58 & 260.05 & 262.45 & 268.88 & 279.34 & 288.28\end{array}$

$\begin{array}{llllll}289.75 & 311.56 & 329.24 & 340.43 & 355.38 & 368.81\end{array}$

$\begin{array}{lllllll}382.50 & 398.78 & 401.48 & 405.83 & 407.12 & 411.39\end{array}$

$\begin{array}{llllll}414.42 & 417.97 & 428.54 & 432.65 & 444.28 & 454.80\end{array}$

$\begin{array}{lllllll}465.31 & 480.82 & 488.94 & 490.55 & 505.04 & 518.68\end{array}$

$\begin{array}{lllllll}526.75 & 531.90 & 548.70 & 555.62 & 583.38 & 588.70\end{array}$

$\begin{array}{lllllll}627.26 & 629.06 & 629.63 & 631.27 & 643.79 & 650.57\end{array}$

$\begin{array}{lllllll}662.85 & 667.24 & 682.65 & 705.02 & 705.87 & 709.37\end{array}$

$\begin{array}{llllll}710.44 & 717.66 & 719.70 & 724.78 & 727.23 & 746.64\end{array}$

$\begin{array}{lllllll}755.56 & 759.80 & 763.79 & 765.66 & 768.77 & 771.45\end{array}$

$\begin{array}{lllllll}790.80 & 799.33 & 824.33 & 832.18 & 861.08 & 862.78\end{array}$

$\begin{array}{lllllll}864.37 & 872.51 & 874.23 & 880.87 & 883.95 & 912.16\end{array}$ $\begin{array}{llllll}939.90 & 941.90 & 949.80 & 950.15 & 978.02 & 982.71\end{array}$ $\begin{array}{llllll}984.09 & 994.01 & 996.27 & 1005.42 & 1006.20 & 1008.12\end{array}$ $\begin{array}{lllllll}1009.24 & 1011.03 & 1013.72 & 1014.24 & 1016.93 & 1024.40\end{array}$ $\begin{array}{lllllll}1039.20 & 1041.08 & 1046.85 & 1052.25 & 1053.43 & 1054.43\end{array}$ $\begin{array}{lllllll}1054.65 & 1056.19 & 1057.48 & 1060.59 & 1064.02 & 1080.97\end{array}$ $\begin{array}{lllllll}1090.77 & 1116.20 & 1116.71 & 1119.29 & 1119.50 & 1124.03\end{array}$ $\begin{array}{lllllll}1124.64 & 1127.32 & 1128.49 & 1168.83 & 1196.61 & 1198.87\end{array}$ $\begin{array}{lllllll}1199.79 & 1200.30 & 1200.36 & 1204.85 & 1220.40 & 1225.65\end{array}$ $\begin{array}{lllllll}1228.64 & 1231.00 & 1231.36 & 1233.29 & 1235.52 & 1290.24\end{array}$ $\begin{array}{lllllll}1294.96 & 1314.74 & 1324.36 & 1326.30 & 1329.59 & 1332.75\end{array}$ $\begin{array}{lllllll}1332.95 & 1336.83 & 1364.03 & 1369.65 & 1374.19 & 1375.13\end{array}$ $\begin{array}{llllllll}1378.98 & 1382.88 & 1393.74 & 1401.71 & 1419.37 & 1427.41\end{array}$ $\begin{array}{lllllll}1432.90 & 1462.62 & 1466.10 & 1468.97 & 1475.50 & 1475.94\end{array}$ $\begin{array}{lllllll}1478.49 & 1480.58 & 1490.34 & 1492.66 & 1497.48 & 1501.74\end{array}$ $\begin{array}{lllllll}1506.34 & 1527.60 & 1529.10 & 1529.59 & 1529.98 & 1532.03\end{array}$ $\begin{array}{llllllll}1533.91 & 1548.59 & 1555.40 & 1625.74 & 1626.84 & 1628.60\end{array}$ $\begin{array}{lllllll}1630.16 & 1645.56 & 1648.03 & 1648.52 & 1649.02 & 1848.75\end{array}$ $\begin{array}{lllllll}1890.41 & 3033.01 & 3045.52 & 3047.39 & 3048.96 & 3064.13\end{array}$ $\begin{array}{lllllll}3066.68 & 3071.65 & 3075.95 & 3093.98 & 3098.51 & 3107.09\end{array}$ $\begin{array}{lllllll}3116.15 & 3127.87 & 3139.43 & 3141.61 & 3160.18 & 3172.78\end{array}$ $\begin{array}{lllllll}3174.89 & 3176.55 & 3177.05 & 3177.80 & 3179.55 & 3182.47\end{array}$ $\begin{array}{lllllll}3183.42 & 3186.94 & 3189.83 & 3189.93 & 3192.62 & 3198.79\end{array}$ $\begin{array}{llllll}3199.96 & 3201.38 & 3203.23 & 3206.69 & 3209.43 & 3211.38\end{array}$ $3218.40 \quad 3222.70 \quad 3238.36$

\begin{tabular}{|c|c|c|c|c|c|}
\hline \multicolumn{5}{|l|}{ I-TS } & \multirow[b]{2}{*}{36.97} \\
\hline-456.25 & 15.97 & 19.05 & 30.66 & 34.36 & \\
\hline 40.68 & 51.48 & 55.87 & 58.43 & 64.21 & 69.48 \\
\hline 71.77 & 77.85 & 86.72 & 94.24 & $98.38 \quad 1$ & 100.64 \\
\hline 113.22 & 114.44 & 119.06 & $\begin{array}{ll}6 & 130.18\end{array}$ & $\begin{array}{ll}8 & 135.25\end{array}$ & $5 \quad 140.56$ \\
\hline 152.72 & 157.75 & 165.78 & $\begin{array}{l}374.69 \\
\end{array}$ & $\begin{array}{ll}9 & 182.41\end{array}$ & 1188.47 \\
\hline 201.15 & 209.53 & 217.67 & $7 \quad 221.09$ & 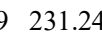 & 237.91 \\
\hline 240.31 & 257.23 & 261.93 & 3268.35 & $\begin{array}{l}5 \quad 277.24 \\
\end{array}$ & $4 \quad 282.45$ \\
\hline 293.16 & 304.10 & 316.53 & 333.46 & $\begin{array}{ll}6 & 338.73\end{array}$ & $3 \quad 346.64$ \\
\hline 355.59 & 376.67 & 379.07 & $\begin{array}{l}785.09 \\
78\end{array}$ & 9406.71 & $1 \quad 410.25$ \\
\hline 411.83 & 416.48 & 417.74 & $\begin{array}{l}4 \\
+\end{array} 19.30$ & $0 \quad 436.66$ & $6 \quad 441.84$ \\
\hline
\end{tabular}

$\begin{array}{llllll}462.70 & 473.76 & 482.72 & 489.07 & 493.89 & 499.59\end{array}$ $\begin{array}{lllllll}522.61 & 529.21 & 531.33 & 551.47 & 584.68 & 592.16\end{array}$ $\begin{array}{lllllll}626.07 & 627.56 & 629.55 & 632.11 & 637.63 & 658.37\end{array}$ $\begin{array}{lllllll}664.06 & 673.56 & 678.75 & 706.09 & 707.63 & 708.78\end{array}$ $\begin{array}{llllll}712.25 & 716.88 & 717.41 & 718.08 & 722.53 & 724.78\end{array}$ $\begin{array}{lllllll}745.39 & 753.44 & 759.76 & 762.56 & 764.50 & 766.54\end{array}$ $\begin{array}{lllllll}786.27 & 819.40 & 826.86 & 840.27 & 860.34 & 863.41\end{array}$ $\begin{array}{lllllll}865.62 & 866.40 & 871.95 & 875.97 & 886.92 & 911.46\end{array}$ $\begin{array}{lllllll}940.07 & 941.00 & 943.98 & 951.05 & 980.51 & 982.02\end{array}$ $\begin{array}{llllll}983.12 & 985.26 & 997.05 & 999.34 & 1000.03 & 1001.52\end{array}$ $\begin{array}{lllllll}1004.02 & 1006.60 & 1008.65 & 1015.23 & 1016.96 & 1018.02\end{array}$ $\begin{array}{lllllll}1024.22 & 1032.06 & 1040.25 & 1044.12 & 1051.64 & 1053.31\end{array}$ $\begin{array}{llllllll}1054.69 & 1055.46 & 1056.07 & 1058.98 & 1061.37 & 1078.63\end{array}$ $\begin{array}{lllllll}1089.65 & 1113.27 & 1114.89 & 1116.82 & 1120.33 & 1122.02\end{array}$ $\begin{array}{lllllll}1125.11 & 1126.15 & 1126.77 & 1187.27 & 1197.23 & 1198.90\end{array}$ $\begin{array}{lllllll}1199.46 & 1200.28 & 1202.10 & 1212.37 & 1222.19 & 1224.98\end{array}$ $\begin{array}{lllllll}1226.69 & 1228.93 & 1231.83 & 1237.30 & 1246.47 & 1276.75\end{array}$ $\begin{array}{lllllll}1309.18 & 1318.79 & 1325.77 & 1326.26 & 1331.90 & 1332.11\end{array}$ $\begin{array}{lllllll}1335.27 & 1335.66 & 1364.87 & 1373.21 & 1374.15 & 1379.04\end{array}$ $\begin{array}{lllllll}1383.07 & 1387.45 & 1398.45 & 1406.49 & 1419.45 & 1424.65\end{array}$ $\begin{array}{llllllll}1433.78 & 1462.37 & 1467.47 & 1474.40 & 1475.06 & 1477.24\end{array}$ $\begin{array}{lllllll}1478.73 & 1479.95 & 1490.30 & 1496.19 & 1498.20 & 1501.08\end{array}$ $\begin{array}{llllllll}1505.53 & 1511.68 & 1521.73 & 1528.99 & 1529.30 & 1529.92\end{array}$ $\begin{array}{lllllll}1533.96 & 1542.79 & 1579.26 & 1627.62 & 1628.10 & 1629.08\end{array}$ $\begin{array}{lllllll}1630.78 & 1647.24 & 1647.59 & 1647.68 & 1648.89 & 1798.78\end{array}$ $\begin{array}{lllllll}2034.13 & 3038.32 & 3039.93 & 3041.40 & 3042.21 & 3058.06\end{array}$ $\begin{array}{lllllll}3062.08 & 3078.41 & 3084.18 & 3089.97 & 3092.59 & 3118.00\end{array}$ $\begin{array}{llllll}3120.82 & 3124.30 & 3125.00 & 3151.56 & 3165.82 & 3166.59\end{array}$ $\begin{array}{lllllll}3174.60 & 3176.39 & 3176.41 & 3176.48 & 3181.30 & 3185.92\end{array}$ $\begin{array}{lllllll}3186.65 & 3188.68 & 3189.11 & 3191.84 & 3199.03 & 3199.22\end{array}$ $\begin{array}{llllll}3199.87 & 3203.66 & 3205.09 & 3210.15 & 3213.14 & 3214.32\end{array}$ $3217.42 \quad 3219.33 \quad 3249.13$

\footnotetext{
$\mathbf{J}$

$\begin{array}{llllll}15.91 & 20.83 & 27.16 & 31.75 & 32.62 & 44.03\end{array}$ $\begin{array}{llllll}46.19 & 56.20 & 60.61 & 64.17 & 66.13 & 71.89\end{array}$ $\begin{array}{lllllll}79.68 & 90.20 & 92.42 & 97.70 & 101.79 & 120.32\end{array}$ $\begin{array}{llllll}126.18 & 140.27 & 153.59 & 160.83 & 162.59 & 166.22\end{array}$ $\begin{array}{lllllll}178.30 & 184.81 & 195.71 & 206.61 & 216.65 & 223.58\end{array}$ $\begin{array}{lllllll}227.26 & 228.79 & 231.75 & 251.00 & 258.60 & 262.46\end{array}$ $\begin{array}{lllllll}264.83 & 274.74 & 283.71 & 296.26 & 300.39 & 331.97\end{array}$ $\begin{array}{llllll}343.26 & 351.05 & 371.38 & 373.72 & 380.83 & 402.00\end{array}$ $\begin{array}{llllll}409.91 & 412.66 & 414.16 & 416.13 & 416.45 & 439.60\end{array}$ $\begin{array}{lllllll}442.77 & 455.05 & 466.83 & 478.26 & 489.39 & 491.06\end{array}$ $\begin{array}{lllllll}495.14 & 523.24 & 527.73 & 529.90 & 549.68 & 581.05\end{array}$ $\begin{array}{lllllll}626.49 & 627.31 & 628.18 & 630.25 & 636.85 & 655.02\end{array}$ $\begin{array}{lllllll}667.49 & 672.32 & 673.87 & 706.56 & 707.68 & 711.79\end{array}$ $\begin{array}{llllll}712.18 & 714.33 & 718.23 & 721.46 & 722.50 & 742.72\end{array}$ $\begin{array}{lllllll}753.40 & 760.56 & 764.04 & 766.04 & 767.86 & 827.53\end{array}$ $\begin{array}{lllllll}831.86 & 839.47 & 859.23 & 867.71 & 869.40 & 871.49\end{array}$ $\begin{array}{lllllll}872.55 & 877.89 & 892.54 & 917.40 & 926.00 & 942.61\end{array}$ $\begin{array}{lllllll}944.82 & 948.90 & 950.41 & 980.87 & 986.18 & 988.00\end{array}$ $\begin{array}{lllllll}992.12 & 994.85 & 1004.19 & 1004.59 & 1007.40 & 1008.97\end{array}$ $\begin{array}{lllllll}1013.62 & 1015.16 & 1016.83 & 1020.58 & 1029.29 & 1031.31\end{array}$ $\begin{array}{lllllll}1036.58 & 1041.11 & 1049.51 & 1050.10 & 1052.19 & 1054.53\end{array}$ $\begin{array}{lllllll}1055.62 & 1057.66 & 1059.80 & 1065.58 & 1086.22 & 1093.77\end{array}$ $\begin{array}{lllllll}1112.34 & 1115.30 & 1116.62 & 1118.55 & 1122.11 & 1124.35\end{array}$ $\begin{array}{lllllll}1125.87 & 1127.80 & 1188.39 & 1198.16 & 1198.94 & 1199.04\end{array}$ $\begin{array}{lllllll}1199.81 & 1203.86 & 1212.18 & 1221.64 & 1225.39 & 1230.06\end{array}$ $\begin{array}{llllll}1230.59 & 1232.42 & 1238.40 & 1253.36 & 1299.44 & 1319.33\end{array}$
} 
$\begin{array}{llllll}1325.27 & 1325.92 & 1330.93 & 1331.59 & 1334.05 & 1335.81\end{array}$ $\begin{array}{lllllll}1365.60 & 1374.31 & 1376.06 & 1379.93 & 1380.73 & 1386.95\end{array}$ $\begin{array}{lllllll}1398.51 & 1408.20 & 1416.75 & 1421.45 & 1433.46 & 1466.42\end{array}$ $\begin{array}{lllllll}1471.61 & 1474.45 & 1476.53 & 1477.57 & 1477.94 & 1479.01\end{array}$ $\begin{array}{lllllll}1492.15 & 1494.89 & 1496.90 & 1500.49 & 1506.01 & 1513.67\end{array}$ $\begin{array}{lllllll}1523.64 & 1527.72 & 1529.66 & 1532.12 & 1534.84 & 1539.88\end{array}$ $\begin{array}{llllllll}1590.78 & 1627.74 & 1627.87 & 1628.77 & 1630.17 & 1646.50\end{array}$ $\begin{array}{lllllll}1646.76 & 1648.11 & 1648.99 & 1770.17 & 3029.45 & 3033.05\end{array}$ $\begin{array}{lllllll}3038.21 & 3039.18 & 3051.67 & 3058.65 & 3061.05 & 3068.37\end{array}$ $\begin{array}{lllllll}3078.17 & 3085.27 & 3106.26 & 3116.80 & 3118.92 & 3121.60\end{array}$ $\begin{array}{llllll}3129.82 & 3141.68 & 3150.79 & 3157.88 & 3173.64 & 3174.01\end{array}$ $\begin{array}{lllllll}3174.10 & 3174.59 & 3181.97 & 3183.62 & 3185.62 & 3186.65\end{array}$ $\begin{array}{lllllll}3191.98 & 3194.09 & 3194.64 & 3197.04 & 3199.98 & 3201.96\end{array}$ 3202.603204 .113204 .753206 .953211 .693212 .51

K

$\begin{array}{llllll}11.83 & 19.50 & 26.84 & 30.36 & 34.15 & 37.54\end{array}$

$\begin{array}{llllll}41.36 & 44.76 & 51.35 & 56.54 & 67.53 & 71.72\end{array}$

$\begin{array}{lllllll}77.02 & 81.14 & 86.40 & 92.27 & 102.84 & 110.89\end{array}$ $\begin{array}{llllll}120.63 & 125.28 & 137.74 & 152.40 & 160.74 & 165.30\end{array}$ $\begin{array}{lllllll}167.27 & 170.44 & 184.58 & 190.43 & 193.85 & 213.61\end{array}$ $\begin{array}{lllllll}218.00 & 220.98 & 224.17 & 235.14 & 258.29 & 260.79\end{array}$ $\begin{array}{llllll}262.15 & 269.90 & 279.63 & 282.61 & 290.32 & 311.09\end{array}$ $\begin{array}{lllllll}329.47 & 346.50 & 367.48 & 369.84 & 383.73 & 398.72\end{array}$ $\begin{array}{lllllll}409.12 & 409.78 & 415.29 & 417.35 & 426.14 & 440.08\end{array}$ $\begin{array}{lllllll}446.53 & 452.24 & 464.61 & 472.99 & 491.12 & 491.86\end{array}$ $\begin{array}{lllllll}496.62 & 521.96 & 527.39 & 529.07 & 544.67 & 565.70\end{array}$ $\begin{array}{llllll}584.42 & 623.66 & 627.10 & 627.61 & 629.44 & 637.51\end{array}$ $\begin{array}{lllllll}647.54 & 660.82 & 674.31 & 677.57 & 705.80 & 707.50\end{array}$ $\begin{array}{llllll}712.01 & 713.41 & 716.17 & 721.46 & 723.96 & 725.81\end{array}$ $\begin{array}{llllll}759.46 & 760.73 & 763.34 & 766.83 & 768.22 & 775.53\end{array}$ $\begin{array}{lllllll}793.26 & 826.38 & 831.70 & 863.03 & 863.15 & 868.89\end{array}$ $\begin{array}{llllll}872.79 & 879.29 & 880.54 & 890.38 & 916.87 & 939.36\end{array}$ $\begin{array}{lllllll}942.94 & 944.27 & 950.49 & 951.55 & 979.91 & 981.34\end{array}$ $\begin{array}{llllll}986.09 & 994.72 & 995.18 & 1002.23 & 1007.14 & 1008.65\end{array}$ $\begin{array}{lllllll}1010.55 & 1011.84 & 1013.99 & 1014.89 & 1035.30 & 1037.88\end{array}$ $\begin{array}{lllllll}1041.65 & 1047.96 & 1051.64 & 1051.94 & 1053.64 & 1054.38\end{array}$ $\begin{array}{lllllll}1058.24 & 1060.24 & 1061.98 & 1069.44 & 1084.14 & 1091.13\end{array}$ $\begin{array}{lllllll}1111.98 & 1116.46 & 1116.52 & 1117.15 & 1124.80 & 1125.07\end{array}$ $\begin{array}{lllllll}1127.01 & 1128.20 & 1195.47 & 1198.75 & 1198.87 & 1199.22\end{array}$ $\begin{array}{lllllll}1199.39 & 1208.28 & 1216.65 & 1221.50 & 1224.35 & 1226.75\end{array}$ $\begin{array}{lllllll}1234.05 & 1236.12 & 1237.16 & 1286.79 & 1298.49 & 1316.56\end{array}$ $\begin{array}{lllllll}1323.81 & 1325.24 & 1330.15 & 1330.89 & 1334.16 & 1335.20\end{array}$ $\begin{array}{lllllll}1365.61 & 1367.48 & 1376.42 & 1379.09 & 1383.16 & 1384.08\end{array}$ $\begin{array}{lllllll}1395.34 & 1404.67 & 1417.83 & 1429.38 & 1430.37 & 1453.76\end{array}$ $\begin{array}{lllllll}1466.03 & 1469.58 & 1473.81 & 1476.22 & 1478.32 & 1478.75\end{array}$ $\begin{array}{lllllll}1490.20 & 1494.07 & 1495.99 & 1502.79 & 1504.67 & 1516.81\end{array}$ $\begin{array}{lllllll}1526.75 & 1527.27 & 1531.82 & 1532.06 & 1536.32 & 1548.03\end{array}$ $\begin{array}{lllllll}1556.35 & 1626.40 & 1626.76 & 1628.83 & 1629.75 & 1645.17\end{array}$ $\begin{array}{lllllll}1647.22 & 1647.54 & 1648.39 & 1857.70 & 3029.05 & 3036.41\end{array}$ $\begin{array}{lllllll}3040.83 & 3042.50 & 3052.57 & 3060.64 & 3065.42 & 3067.47\end{array}$ $\begin{array}{lllllll}3078.05 & 3094.48 & 3105.00 & 3111.07 & 3114.60 & 3127.50\end{array}$ $\begin{array}{lllllll}3130.90 & 3142.55 & 3146.85 & 3156.12 & 3172.02 & 3172.65\end{array}$ $\begin{array}{llllll}3173.34 & 3174.06 & 3175.18 & 3176.21 & 3182.51 & 3184.70\end{array}$ $\begin{array}{llllll}3184.86 & 3188.35 & 3192.17 & 3196.10 & 3196.63 & 3200.13\end{array}$

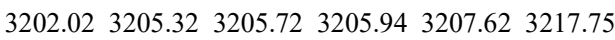

\begin{tabular}{|c|c|c|c|}
\hline K-TS & & & \\
\hline-242.73 & 12.41 & 21.98 & 23.92 \\
\hline
\end{tabular}

$\begin{array}{llllll}34.01 & 38.11 & 43.24 & 49.23 & 52.73 & 61.94\end{array}$ $\begin{array}{llllll}65.19 & 68.84 & 77.47 & 80.59 & 91.50 & 100.45\end{array}$ $\begin{array}{llllll}102.63 & 120.93 & 132.45 & 135.30 & 157.10 & 163.11\end{array}$ $\begin{array}{llllll}166.47 & 171.16 & 179.66 & 187.38 & 189.66 & 200.39\end{array}$ $\begin{array}{lllllll}214.33 & 217.81 & 222.46 & 225.36 & 245.84 & 257.00\end{array}$ $\begin{array}{lllllll}261.12 & 263.22 & 275.26 & 281.00 & 289.95 & 294.35\end{array}$ $\begin{array}{lllllll}310.87 & 335.43 & 349.76 & 367.93 & 383.08 & 397.72\end{array}$ $\begin{array}{lllllll}406.44 & 408.34 & 413.63 & 414.77 & 424.00 & 431.25\end{array}$ $\begin{array}{lllllll}437.23 & 443.85 & 453.21 & 463.98 & 473.46 & 486.28\end{array}$ $\begin{array}{llllll}490.44 & 492.24 & 494.12 & 524.29 & 527.83 & 531.79\end{array}$ $\begin{array}{lllllll}544.19 & 581.80 & 628.23 & 628.40 & 628.83 & 629.97\end{array}$ $\begin{array}{lllllll}635.65 & 659.31 & 669.68 & 673.50 & 703.97 & 706.49\end{array}$ $\begin{array}{llllll}710.62 & 712.06 & 715.16 & 717.91 & 723.55 & 724.70\end{array}$ $\begin{array}{llllll}727.97 & 759.75 & 760.89 & 762.98 & 765.73 & 766.70\end{array}$ $\begin{array}{llllll}804.74 & 825.00 & 829.65 & 861.54 & 864.13 & 867.15\end{array}$ $\begin{array}{lllllll}873.95 & 874.71 & 877.53 & 890.56 & 917.17 & 923.14\end{array}$ $\begin{array}{lllllll}937.41 & 940.90 & 947.24 & 948.69 & 977.65 & 982.14\end{array}$ $\begin{array}{lllllll}983.70 & 992.01 & 992.74 & 1002.79 & 1006.85 & 1008.14\end{array}$ $\begin{array}{lllllll}1009.86 & 1011.34 & 1013.82 & 1014.13 & 1025.70 & 1027.48\end{array}$ $\begin{array}{lllllll}1036.63 & 1040.75 & 1048.18 & 1051.39 & 1052.22 & 1053.52\end{array}$ $\begin{array}{lllllll}1054.35 & 1058.40 & 1060.40 & 1068.44 & 1086.14 & 1092.09\end{array}$ $\begin{array}{lllllll}1111.54 & 1114.90 & 1115.48 & 1115.92 & 1123.30 & 1125.15\end{array}$ $\begin{array}{lllllll}1126.80 & 1127.02 & 1196.14 & 1197.99 & 1198.12 & 1198.26\end{array}$ $\begin{array}{lllllll}1199.18 & 1207.40 & 1216.64 & 1222.50 & 1223.79 & 1226.68\end{array}$ $\begin{array}{lllllll}1232.26 & 1232.97 & 1235.89 & 1297.46 & 1299.91 & 1311.54\end{array}$ $\begin{array}{lllllll}1323.35 & 1324.89 & 1328.52 & 1330.39 & 1333.47 & 1335.09\end{array}$ $\begin{array}{lllllll}1365.30 & 1366.95 & 1374.35 & 1375.78 & 1377.39 & 1381.77\end{array}$ $\begin{array}{lllllll}1391.48 & 1400.86 & 1418.81 & 1423.58 & 1432.15 & 1458.92\end{array}$ $\begin{array}{lllllll}1466.08 & 1467.39 & 1473.66 & 1475.81 & 1477.39 & 1478.76\end{array}$ $\begin{array}{lllllll}1490.44 & 1494.74 & 1496.12 & 1503.70 & 1505.59 & 1513.98\end{array}$ $\begin{array}{lllllll}1523.54 & 1527.25 & 1527.62 & 1530.46 & 1533.30 & 1537.59\end{array}$ $\begin{array}{lllllll}1547.77 & 1625.18 & 1626.34 & 1627.12 & 1629.96 & 1644.77\end{array}$ $\begin{array}{lllllll}1646.35 & 1646.92 & 1647.62 & 2053.31 & 3028.80 & 3034.70\end{array}$ $\begin{array}{llllll}3036.98 & 3037.85 & 3051.22 & 3057.43 & 3059.36 & 3066.57\end{array}$ $\begin{array}{llllll}3075.73 & 3089.50 & 3100.81 & 3105.83 & 3108.19 & 3118.31\end{array}$ $\begin{array}{lllllll}3139.20 & 3145.06 & 3154.88 & 3171.64 & 3173.98 & 3174.19\end{array}$ $\begin{array}{llllll}3174.81 & 3175.26 & 3182.36 & 3182.70 & 3183.96 & 3185.15\end{array}$ $\begin{array}{lllllll}3189.74 & 3192.35 & 3193.96 & 3195.97 & 3199.32 & 3200.75\end{array}$

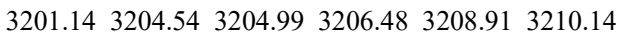

$\mathbf{L}$

$\begin{array}{llllll}15.25 & 18.72 & 20.39 & 33.11 & 34.24 & 39.02\end{array}$ $\begin{array}{lllllll}42.43 & 47.12 & 47.99 & 56.99 & 66.07 & 77.86\end{array}$ $\begin{array}{lllllll}78.85 & 86.96 & 108.78 & 117.36 & 131.83 & 150.84\end{array}$ $\begin{array}{llllll}153.90 & 162.35 & 167.87 & 172.17 & 180.88 & 184.64\end{array}$ $\begin{array}{lllllll}195.13 & 195.51 & 212.18 & 216.47 & 224.17 & 226.71\end{array}$ $\begin{array}{lllllll}246.52 & 253.25 & 257.18 & 264.02 & 273.25 & 283.44\end{array}$ $\begin{array}{lllllll}283.79 & 303.61 & 310.20 & 338.46 & 356.69 & 368.16\end{array}$ $\begin{array}{lllllll}381.15 & 393.88 & 408.43 & 410.69 & 412.90 & 416.21\end{array}$ $\begin{array}{lllllll}423.12 & 431.83 & 438.94 & 446.10 & 457.31 & 470.41\end{array}$ $\begin{array}{lllllll}483.01 & 492.74 & 495.01 & 522.12 & 525.46 & 527.95\end{array}$ $\begin{array}{lllllll}542.96 & 574.82 & 627.19 & 628.75 & 629.06 & 629.46\end{array}$ $\begin{array}{lllllll}630.15 & 658.09 & 671.59 & 677.38 & 708.05 & 708.62\end{array}$ $\begin{array}{lllllll}712.65 & 713.08 & 714.41 & 717.27 & 721.56 & 726.27\end{array}$ $\begin{array}{lllllll}757.70 & 759.90 & 763.17 & 765.57 & 768.92 & 824.29\end{array}$ $\begin{array}{lllllll}828.09 & 845.96 & 864.58 & 868.10 & 869.26 & 870.34\end{array}$ $\begin{array}{lllllll}873.00 & 874.30 & 882.87 & 892.68 & 922.96 & 940.11\end{array}$ $\begin{array}{llllll}942.04 & 943.75 & 945.69 & 982.32 & 984.95 & 986.02\end{array}$ $\begin{array}{llllll}986.26 & 990.93 & 1004.89 & 1007.36 & 1007.99 & 1008.58\end{array}$ $\begin{array}{llllll}1009.41 & 1011.84 & 1014.48 & 1014.87 & 1016.22 & 1037.33\end{array}$ 
$\begin{array}{llllll}1037.67 & 1045.61 & 1049.66 & 1053.10 & 1054.03 & 1054.15\end{array}$ $\begin{array}{lllllll}1056.22 & 1059.65 & 1067.30 & 1088.90 & 1092.63 & 1111.41\end{array}$ $\begin{array}{lllllll}1111.81 & 1114.03 & 1115.00 & 1120.16 & 1124.48 & 1128.08\end{array}$ $\begin{array}{lllllll}1130.17 & 1195.51 & 1197.14 & 1197.99 & 1198.17 & 1198.26\end{array}$ $\begin{array}{lllllll}1203.64 & 1214.40 & 1223.73 & 1224.41 & 1224.63 & 1225.39\end{array}$ $\begin{array}{lllllll}1228.25 & 1232.11 & 1295.65 & 1306.29 & 1324.87 & 1325.45\end{array}$ $\begin{array}{lllllll}1328.80 & 1330.07 & 1331.79 & 1332.97 & 1366.18 & 1368.13\end{array}$ $\begin{array}{lllllll}1369.48 & 1369.58 & 1372.95 & 1379.14 & 1387.54 & 1398.83\end{array}$ $\begin{array}{lllllll}1417.24 & 1418.02 & 1433.24 & 1463.85 & 1464.65 & 1471.61\end{array}$ $\begin{array}{lllllll}1476.18 & 1476.73 & 1476.96 & 1477.74 & 1487.78 & 1495.44\end{array}$ $\begin{array}{lllllll}1495.59 & 1497.76 & 1504.65 & 1508.33 & 1512.78 & 1523.07\end{array}$ $\begin{array}{lllllll}1527.46 & 1527.92 & 1529.75 & 1530.08 & 1550.06 & 1627.14\end{array}$ $\begin{array}{lllllll}1628.36 & 1628.40 & 1629.30 & 1645.27 & 1646.09 & 1648.02\end{array}$ $\begin{array}{lllllll}1648.19 & 3025.38 & 3026.28 & 3027.55 & 3030.80 & 3043.66\end{array}$ $\begin{array}{lllllll}3045.88 & 3047.52 & 3047.95 & 3072.76 & 3079.02 & 3087.88\end{array}$ $\begin{array}{lllllll}3090.29 & 3100.45 & 3101.53 & 3112.60 & 3117.32 & 3149.27\end{array}$ $\begin{array}{llllll}3167.04 & 3168.87 & 3172.11 & 3172.35 & 3173.26 & 3177.96\end{array}$ $\begin{array}{llllll}3178.15 & 3180.67 & 3181.78 & 3187.05 & 3188.27 & 3190.66\end{array}$ $\begin{array}{lllllll}3191.07 & 3194.82 & 3197.73 & 3198.13 & 3199.87 & 3203.17\end{array}$ $3203.82 \quad 3205.043205 .63$

\begin{tabular}{|c|c|c|c|c|c|}
\hline & & & & & \\
\hline 7.22 & 12.25 & 14.05 & 17.78 & 19.39 & 21.28 \\
\hline 22.73 & 23.58 & 25.43 & 27.39 & 30.56 & 32.42 \\
\hline 36.18 & 39.68 & 42.53 & 44.70 & 46.03 & 49.14 \\
\hline 51.31 & 52.29 & 53.86 & 58.52 & 60.82 & 62.52 \\
\hline 63.98 & 67.13 & 71.44 & 74.65 & 76.99 & 80.33 \\
\hline 83.75 & 84.95 & 90.12 & 91.16 & 97.59 & 98.44 \\
\hline 105.40 & 108.63 & 109.64 & $\begin{array}{ll}4 & 112.92\end{array}$ & 2117.31 & $1 \quad 120.70$ \\
\hline 124.02 & 130.95 & 134.26 & $\begin{array}{ll}6 & 141.79\end{array}$ & $9 \quad 143.33$ & $\begin{array}{ll}3 & 146.70\end{array}$ \\
\hline 149.99 & 151.02 & 154.69 & 157.18 & $\begin{array}{ll}8 & 159.88\end{array}$ & $8 \quad 164.94$ \\
\hline 168.42 & 173.13 & 177.43 & 3179.47 & $\begin{array}{ll}7 & 186.24\end{array}$ & $4 \quad 194.12$ \\
\hline 199.70 & 203.64 & 204.19 & 206.55 & $5 \quad 211.27$ & $\begin{array}{ll}7 & 214.19\end{array}$ \\
\hline 219.07 & 223.25 & 224.56 & $6 \quad 228.23$ & 3229.86 & $6 \quad 232.35$ \\
\hline 236.20 & 239.91 & 248.24 & $+\quad 252.05$ & $5 \quad 261.65$ & 265.52 \\
\hline 266.92 & 269.08 & 270.41 & I 274.15 & $5 \quad 277.56$ & $\begin{array}{ll}6 & 282.11\end{array}$ \\
\hline 288.66 & 302.49 & 312.30 & 313.33 & $\begin{array}{ll}3 & 321.79\end{array}$ & $9 \quad 324.98$ \\
\hline 327.82 & 330.38 & 341.96 & $6 \quad 354.92$ & 2360.63 & $3 \quad 368.55$ \\
\hline 376.90 & 381.38 & 394.66 & $5 \quad 398.97$ & $7 \quad 404.10$ & $0 \quad 405.95$ \\
\hline 407.94 & 408.40 & 410.03 & 3412.21 & 1415.22 & 2415.53 \\
\hline 416.42 & 418.94 & 421.29 & 422.36 & $\begin{array}{ll}6 & 424.79\end{array}$ & $9 \quad 426.98$ \\
\hline 432.91 & 438.52 & 446.98 & 3447.41 & $1 \quad 453.42$ & 2460.00 \\
\hline 470.33 & 473.72 & 491.71 & $\begin{array}{l}1497.50 \\
\end{array}$ & $\begin{array}{ll}0 & 497.79\end{array}$ & $9 \quad 499.82$ \\
\hline 515.73 & 518.16 & 520.72 & 2523.83 & 3525.81 & 1527.73 \\
\hline 533.52 & 538.80 & 583.96 & $5 \quad 585.52$ & $2 \quad 622.57$ & $7 \quad 628.12$ \\
\hline 629.28 & 629.74 & 630.37 & $7 \quad 630.73$ & 3631.86 & $6 \quad 632.24$ \\
\hline 632.83 & 632.92 & 634.93 & 3637.95 & $5 \quad 643.82$ & 2662.52 \\
\hline 664.16 & 669.80 & 678.85 & $5 \quad 690.08$ & $8 \quad 691.78$ & $8 \quad 694.98$ \\
\hline 703.20 & 705.31 & 705.71 & 706.72 & 2709.02 & 2713.03 \\
\hline 713.13 & 713.33 & 713.68 & 716.95 & $5 \quad 717.77$ & $7 \quad 718.39$ \\
\hline 720.85 & 721.01 & 724.45 & 726.70 & $\begin{array}{ll}0 & 732.27\end{array}$ & $7 \quad 747.43$ \\
\hline 755.47 & 756.42 & 760.07 & 760.36 & 6763.33 & 3763.52 \\
\hline 768.30 & 769.47 & 770.30 & 772.83 & $\begin{array}{ll}3 & 816.14\end{array}$ & $4 \quad 824.84$ \\
\hline 825.53 & 831.42 & 845.51 & 848.06 & $6 \quad 861.56$ & 6862.53 \\
\hline 865.50 & 866.28 & 866.91 & 868.10 & $\begin{array}{ll}0 & 869.51\end{array}$ & 1871.67 \\
\hline 879.96 & 886.26 & 902.08 & 904.44 & $\begin{array}{ll}4 & 921.88\end{array}$ & 8922.71 \\
\hline 938.97 & 939.33 & 941.26 & 941.67 & $\begin{array}{ll}7 & 943.21\end{array}$ & 1945.58 \\
\hline 948.51 & 950.34 & 954.34 & +962.90 & $\begin{array}{ll}0 & 973.34\end{array}$ & $4 \quad 975.47$ \\
\hline 980.04 & 982.82 & 985.01 & 986.72 & 2990.22 & 2991.59 \\
\hline 992.02 & 995.93 & 996.62 & 997.14 & 41003.49 & 91006.01 \\
\hline
\end{tabular}

$\begin{array}{llllll}1006.84 & 1007.99 & 1008.52 & 1010.44 & 1011.65 & 1012.04\end{array}$ $\begin{array}{lllllll}1012.83 & 1013.46 & 1014.35 & 1015.24 & 1016.66 & 1017.91\end{array}$ $\begin{array}{lllllll}1021.24 & 1022.68 & 1024.41 & 1027.74 & 1030.88 & 1033.14\end{array}$ $\begin{array}{lllllll}1036.05 & 1039.96 & 1041.71 & 1042.52 & 1044.08 & 1046.86\end{array}$ $\begin{array}{lllllll}1049.06 & 1050.78 & 1052.10 & 1052.42 & 1053.09 & 1053.48\end{array}$ $\begin{array}{llllllll}1054.38 & 1054.69 & 1059.23 & 1059.70 & 1061.42 & 1062.36\end{array}$ $\begin{array}{lllllll}1069.58 & 1071.18 & 1089.71 & 1092.63 & 1094.98 & 1106.94\end{array}$ $\begin{array}{lllllll}1108.26 & 1109.03 & 1109.92 & 1110.95 & 1111.78 & 1112.86\end{array}$ $\begin{array}{lllllll}1114.49 & 1114.95 & 1118.09 & 1118.52 & 1118.80 & 1119.75\end{array}$ $\begin{array}{lllllll}1120.59 & 1123.27 & 1125.20 & 1127.63 & 1192.32 & 1193.83\end{array}$ $\begin{array}{lllllll}1195.36 & 1195.99 & 1196.22 & 1196.27 & 1196.41 & 1197.90\end{array}$ $\begin{array}{lllllll}1198.50 & 1198.88 & 1204.11 & 1213.77 & 1216.68 & 1220.13\end{array}$ $\begin{array}{lllllll}1220.55 & 1220.77 & 1223.66 & 1223.82 & 1224.15 & 1224.74\end{array}$ $\begin{array}{lllllll}1225.78 & 1226.23 & 1230.59 & 1235.65 & 1236.59 & 1240.45\end{array}$ $\begin{array}{lllllll}1249.22 & 1287.82 & 1308.52 & 1311.48 & 1317.07 & 1318.09\end{array}$ $\begin{array}{lllllll}1325.02 & 1325.51 & 1327.31 & 1327.97 & 1328.90 & 1330.53\end{array}$ $\begin{array}{lllllll}1332.76 & 1334.70 & 1337.35 & 1340.91 & 1347.74 & 1353.60\end{array}$ $\begin{array}{lllllll}1358.11 & 1360.05 & 1364.88 & 1365.75 & 1367.39 & 1367.65\end{array}$ $\begin{array}{lllllll}1368.08 & 1369.74 & 1372.42 & 1379.11 & 1385.47 & 1387.33\end{array}$ $\begin{array}{lllllll}1395.30 & 1400.02 & 1410.22 & 1411.53 & 1415.79 & 1418.41\end{array}$ $\begin{array}{lllllll}1419.19 & 1421.62 & 1427.39 & 1427.75 & 1455.99 & 1461.44\end{array}$ $\begin{array}{lllllll}1469.85 & 1470.60 & 1473.53 & 1475.71 & 1476.21 & 1477.01\end{array}$ $\begin{array}{lllllll}1477.37 & 1477.80 & 1482.94 & 1486.28 & 1490.11 & 1490.39\end{array}$ $\begin{array}{lllllll}1492.04 & 1493.52 & 1494.45 & 1495.53 & 1497.48 & 1498.61\end{array}$ $\begin{array}{llllllll}1504.59 & 1505.43 & 1508.06 & 1513.16 & 1520.65 & 1521.77\end{array}$ $\begin{array}{lllllll}1523.96 & 1526.93 & 1527.85 & 1527.94 & 1528.26 & 1528.59\end{array}$ $\begin{array}{lllllll}1529.90 & 1530.76 & 1536.16 & 1538.04 & 1556.92 & 1559.01\end{array}$ $\begin{array}{lllllll}1603.18 & 1614.96 & 1622.05 & 1622.15 & 1624.29 & 1625.15\end{array}$ $\begin{array}{lllllll}1625.36 & 1629.34 & 1630.05 & 1630.50 & 1641.31 & 1641.76\end{array}$ $\begin{array}{lllllll}1644.09 & 1645.38 & 1646.18 & 1646.38 & 1648.19 & 1649.31\end{array}$ $\begin{array}{lllllll}1727.11 & 1784.75 & 3026.40 & 3027.53 & 3030.02 & 3030.53\end{array}$ $\begin{array}{lllllll}3032.08 & 3032.50 & 3043.99 & 3045.32 & 3046.96 & 3047.68\end{array}$ $\begin{array}{lllllll}3059.90 & 3061.19 & 3064.01 & 3064.75 & 3072.07 & 3074.09\end{array}$ $\begin{array}{lllllll}3075.10 & 3075.87 & 3086.00 & 3088.75 & 3098.74 & 3101.68\end{array}$ $\begin{array}{llllll}3108.95 & 3111.48 & 3111.92 & 3115.85 & 3117.69 & 3126.46\end{array}$ $\begin{array}{lllllll}3128.47 & 3142.58 & 3146.65 & 3147.83 & 3148.07 & 3150.61\end{array}$ $\begin{array}{llllll}3157.27 & 3159.05 & 3164.81 & 3165.79 & 3166.73 & 3168.11\end{array}$ $\begin{array}{lllllll}3169.61 & 3172.85 & 3172.88 & 3173.79 & 3173.90 & 3174.92\end{array}$ $\begin{array}{lllllll}3176.69 & 3181.77 & 3181.85 & 3182.76 & 3183.76 & 3184.41\end{array}$ $\begin{array}{lllllll}3185.50 & 3189.09 & 3190.45 & 3191.40 & 3193.08 & 3193.23\end{array}$ $\begin{array}{lllllll}3195.83 & 3196.27 & 3197.51 & 3197.88 & 3199.13 & 3199.97\end{array}$ $\begin{array}{lllllll}3200.46 & 3202.80 & 3204.32 & 3205.00 & 3206.89 & 3210.46\end{array}$ $\begin{array}{lllllll}3210.53 & 3216.73 & 3219.01 & 3229.96 & 3238.70 & 3249.01\end{array}$

\begin{tabular}{|c|c|c|c|c|c|}
\hline $\mathbf{N}$ & & & & & \\
\hline 13.96 & 14.18 & 16.93 & 19.24 & 24.35 & 25.90 \\
\hline 26.67 & 27.55 & 30.86 & 31.57 & 33.49 & 34.91 \\
\hline 39.03 & 40.59 & 41.97 & 44.67 & 46.88 & 50.41 \\
\hline 54.68 & 56.93 & 59.32 & 60.47 & 61.80 & 64.04 \\
\hline 66.42 & 68.67 & 70.79 & 72.23 & 76.76 & 79.01 \\
\hline 80.11 & 82.92 & 85.62 & 88.33 & 90.63 & 93.60 \\
\hline 98.06 & 105.54 & 110.10 & 117.29 & 117.91 & 122.38 \\
\hline 123.26 & 127.68 & 130.70 & 137.28 & $\begin{array}{ll}8 & 138.81\end{array}$ & 1144.88 \\
\hline 152.96 & 156.04 & 158.17 & 164.31 & $\begin{array}{ll}1 & 167.25\end{array}$ & 169.17 \\
\hline 178.78 & 179.25 & 180.29 & 187.25 & $\begin{array}{ll}5 & 189.67\end{array}$ & 194.37 \\
\hline 198.27 & 200.84 & 201.47 & 205.56 & $\begin{array}{ll}6 & 212.50\end{array}$ & 213.54 \\
\hline 215.60 & 223.38 & 228.33 & 229.38 & 234.42 & $2 \quad 235.86$ \\
\hline 239.15 & 240.85 & 244.97 & 247.87 & 251.03 & 253.00 \\
\hline 255.03 & 256.50 & 260.03 & 263.83 & 265.24 & 277.02 \\
\hline 279.08 & 296.97 & 301.38 & 309.70 & 315.34 & $4 \quad 319.20$ \\
\hline
\end{tabular}


$\begin{array}{llllll}326.69 & 332.16 & 347.97 & 349.86 & 362.90 & 363.33\end{array}$ $\begin{array}{lllllll}374.19 & 381.67 & 390.06 & 395.34 & 397.46 & 403.30\end{array}$ $\begin{array}{lllllll}405.18 & 406.21 & 406.68 & 407.23 & 408.68 & 409.40\end{array}$ $\begin{array}{lllllll}412.61 & 413.36 & 416.60 & 417.04 & 418.26 & 421.07\end{array}$ $\begin{array}{lllllll}434.91 & 438.66 & 442.86 & 444.61 & 451.95 & 454.50\end{array}$ $\begin{array}{lllllll}455.77 & 457.38 & 465.84 & 475.81 & 491.32 & 498.04\end{array}$ $\begin{array}{lllllll}499.16 & 500.84 & 506.66 & 509.78 & 516.58 & 519.45\end{array}$ $\begin{array}{lllllll}521.06 & 521.63 & 534.34 & 543.48 & 544.57 & 545.23\end{array}$ $\begin{array}{lllllll}548.11 & 560.43 & 587.19 & 592.95 & 628.43 & 629.80\end{array}$ $\begin{array}{lllllll}630.78 & 631.24 & 631.96 & 632.27 & 632.40 & 633.55\end{array}$ $\begin{array}{lllllll}633.66 & 640.02 & 642.72 & 649.46 & 659.53 & 661.03\end{array}$ $\begin{array}{lllllll}671.54 & 682.57 & 685.79 & 692.24 & 692.93 & 702.80\end{array}$ $\begin{array}{llllll}703.52 & 704.56 & 705.49 & 710.23 & 711.14 & 712.23\end{array}$ $\begin{array}{llllll}712.75 & 714.67 & 715.07 & 716.90 & 718.04 & 718.86\end{array}$ $\begin{array}{llllll}719.23 & 721.81 & 730.10 & 731.50 & 751.25 & 758.07\end{array}$ $\begin{array}{llllll}758.92 & 760.05 & 760.23 & 764.07 & 764.57 & 770.47\end{array}$ $\begin{array}{llllll}770.68 & 779.12 & 779.92 & 794.41 & 823.78 & 833.65\end{array}$ $\begin{array}{lllllll}839.74 & 842.75 & 852.84 & 855.59 & 856.43 & 857.63\end{array}$ $\begin{array}{lllllll}863.13 & 863.93 & 864.35 & 865.56 & 867.20 & 870.82\end{array}$ $\begin{array}{lllllll}879.14 & 879.17 & 886.94 & 905.29 & 921.70 & 924.08\end{array}$ $\begin{array}{lllllll}928.07 & 933.74 & 940.03 & 941.04 & 942.84 & 945.68\end{array}$ $\begin{array}{llllll}947.20 & 970.92 & 973.08 & 973.69 & 977.91 & 979.64\end{array}$ $\begin{array}{llllll}983.61 & 984.67 & 989.58 & 990.37 & 990.55 & 996.86\end{array}$ $\begin{array}{llllll}997.70 & 1002.80 & 1004.03 & 1004.07 & 1006.24 & 1008.42\end{array}$ $\begin{array}{llllll}1008.75 & 1011.04 & 1011.96 & 1012.23 & 1012.96 & 1015.29\end{array}$ $\begin{array}{lllllll}1015.79 & 1016.21 & 1018.56 & 1019.10 & 1027.37 & 1028.99\end{array}$ $\begin{array}{llllll}1029.96 & 1031.40 & 1036.67 & 1040.21 & 1042.29 & 1043.93\end{array}$ $\begin{array}{lllllll}1046.93 & 1049.12 & 1050.13 & 1050.24 & 1051.70 & 1052.64\end{array}$ $\begin{array}{lllllll}1052.97 & 1054.34 & 1054.38 & 1054.81 & 1055.00 & 1057.70\end{array}$ $\begin{array}{lllllll}1058.99 & 1061.12 & 1065.70 & 1066.89 & 1070.30 & 1076.11\end{array}$ $\begin{array}{lllllll}1085.44 & 1096.84 & 1107.36 & 1107.58 & 1109.52 & 1109.55\end{array}$ $\begin{array}{llllll}1111.90 & 1114.46 & 1115.15 & 1115.68 & 1117.24 & 1118.39\end{array}$ $\begin{array}{llllll}1118.49 & 1118.70 & 1119.17 & 1120.26 & 1122.18 & 1122.51\end{array}$ $\begin{array}{lllllll}1138.32 & 1194.24 & 1194.71 & 1195.60 & 1195.61 & 1197.29\end{array}$ $\begin{array}{llllll}1197.50 & 1198.27 & 1198.61 & 1198.97 & 1200.75 & 1206.26\end{array}$ $\begin{array}{lllllll}1207.28 & 1212.59 & 1217.47 & 1222.05 & 1222.35 & 1223.00\end{array}$ $\begin{array}{llllll}1224.16 & 1226.58 & 1226.78 & 1227.13 & 1230.29 & 1232.49\end{array}$ $\begin{array}{lllllll}1235.47 & 1236.47 & 1237.68 & 1268.26 & 1295.37 & 1307.29\end{array}$ $\begin{array}{lllllll}1311.92 & 1318.93 & 1319.40 & 1320.17 & 1322.84 & 1323.33\end{array}$ $\begin{array}{lllllll}1325.42 & 1327.25 & 1327.60 & 1328.75 & 1334.70 & 1335.74\end{array}$ $\begin{array}{lllllll}1349.61 & 1350.60 & 1355.45 & 1360.36 & 1361.43 & 1364.16\end{array}$ $\begin{array}{lllllll}1365.41 & 1366.21 & 1366.45 & 1367.31 & 1367.66 & 1369.37\end{array}$ $\begin{array}{lllllll}1376.31 & 1379.48 & 1389.70 & 1398.77 & 1399.59 & 1409.02\end{array}$ $\begin{array}{lllllll}1417.57 & 1418.01 & 1424.09 & 1428.58 & 1428.76 & 1434.32\end{array}$ $\begin{array}{lllllll}1437.07 & 1459.99 & 1466.18 & 1468.60 & 1470.78 & 1471.55\end{array}$ $\begin{array}{lllllll}1473.61 & 1474.30 & 1474.39 & 1475.15 & 1476.55 & 1476.79\end{array}$ $\begin{array}{lllllll}1484.02 & 1484.25 & 1485.15 & 1489.70 & 1490.29 & 1497.07\end{array}$ $1497.98 \quad 1498.69 \quad 1500.76 \quad 1504.43 \quad 1506.89 \quad 1514.17$ $\begin{array}{lllllll}1520.50 & 1522.79 & 1525.65 & 1525.98 & 1526.71 & 1527.14\end{array}$ $\begin{array}{lllllll}1527.36 & 1527.44 & 1527.64 & 1530.06 & 1531.02 & 1535.79\end{array}$ $\begin{array}{lllllll}1537.03 & 1544.51 & 1546.65 & 1568.19 & 1597.32 & 1622.82\end{array}$ $\begin{array}{llllll}1623.46 & 1624.29 & 1625.09 & 1625.52 & 1626.05 & 1629.38\end{array}$ $\begin{array}{lllllll}1629.45 & 1641.24 & 1641.84 & 1643.11 & 1643.59 & 1645.57\end{array}$ $\begin{array}{lllllll}1645.59 & 1648.34 & 1648.53 & 1688.30 & 1740.97 & 1908.91\end{array}$ $\begin{array}{lllllll}1925.73 & 2990.13 & 3009.31 & 3024.10 & 3025.74 & 3028.37\end{array}$ $\begin{array}{lllllll}3034.63 & 3035.07 & 3039.27 & 3040.36 & 3042.33 & 3046.00\end{array}$ $3050.013064 .86 \quad 3066.60 \quad 3069.93 \quad 3081.36 \quad 3083.50$ $3083.94 \quad 3084.25 \quad 3092.48 \quad 3095.65 \quad 3095.84 \quad 3097.23$ $\begin{array}{lllllll}3098.50 & 3098.53 & 3103.19 & 3104.50 & 3105.10 & 3109.62\end{array}$ $3125.62 \quad 3127.84 \quad 3146.19 \quad 3153.94 \quad 3154.80 \quad 3158.15$ $\begin{array}{llllll}3159.83 & 3163.84 & 3163.99 & 3164.02 & 3164.95 & 3169.92\end{array}$ $\begin{array}{lllllll}3170.54 & 3171.88 & 3172.21 & 3172.74 & 3173.67 & 3177.75\end{array}$ $\begin{array}{lllllll}3177.84 & 3179.71 & 3180.23 & 3180.98 & 3181.54 & 3181.85\end{array}$ $\begin{array}{lllllll}3183.21 & 3188.49 & 3188.54 & 3189.94 & 3193.37 & 3194.09\end{array}$ $\begin{array}{lllllll}3196.30 & 3196.61 & 3197.19 & 3197.57 & 3198.12 & 3201.85\end{array}$ $\begin{array}{lllllll}3204.99 & 3205.43 & 3205.66 & 3205.79 & 3213.73 & 3215.61\end{array}$ $\begin{array}{llllll}3218.50 & 3224.73 & 3233.32 & 3246.32 & 3248.41\end{array}$ 